\title{
PUSHING THE BOUNDARIES: RELATIONSHIPS WITH
} ADOLESCENTS

\author{
by \\ Julia Margaret McLean
}
A thesis submitted to the Victoria University of Wellington in partial fulfilment of the requirements for the degree of Master of Arts (Applied)

Victoria University of Wellington 2007 


\section{Abstract}

Therapeutic relationships are central to mental health nursing. The nurse's role in maintaining professional yet therapeutic boundaries within this relationship can be challenging. When therapeutic boundaries are breeched within the nurse adolescent relationship the adolescent's safety within this relationship is compromised. There is currently limited literature on how nurses are managing professional boundaries in relationships with adolescents in this setting. The agdolescent's nature is to push boundaries; therefore the nurse needs to be acutely aware of this boundary pushing in everyday practice settings. For the safety of the adolescent and the nurse it is vital the nurse understands her role in managing the professional boundary.

This thesis explores, through the use of narrative inquiry, four adolescent mental health nurses' experiences of assessing, understanding and maintaining therapeutic boundaries with adolescents in a mental health setting in New Zealand. The unique and specific implications for adolescent mental health nursing are discussed.

Three key themes emerged from the analysis and findings: the importance of the nurse clarifying his/her role; the learning that occurs throughout the practice journey; and the role of the nurse in keeping the adolescent and the nurse safe.

These findings highlight the importance of clinical supervision and open communication with senior nurses and mentors, which assist the nurse in monitoring practice. When nurses do not have sufficient knowledge of the fundamental principles of adolescent mental health nursing; such as knowledge and skills in both adolescent development and psychodynamic nursing, they are at risk of boundary crossings. Recommendations from this research include more emphasis on psychodynamic nursing principles in nursing education and nursing practice. There is a need for specialised education for nurses in child 
and adolescent mental health nursing. Nursing entry to practice programmes for new graduate nurses working in mental health could assist in providing this. There is a call for further research into therapeutic relationships and professional boundaries in this complex nursing specialty.

Keywords: Adolescent mental health nursing, therapeutic boundaries, narrative inquiry. 


\section{Table of Contents}

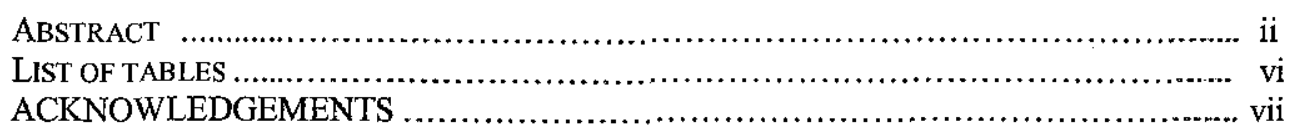

CHAPTER 1 ...................................................................................... 1

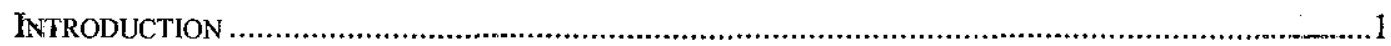

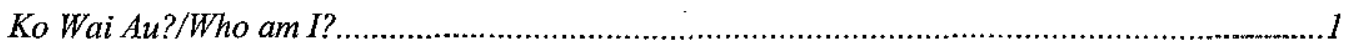

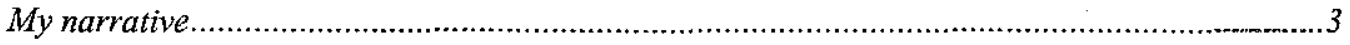

The therapeutic relationship and professional boundaries ............................................... 7

Nursing education in New Zealand........................................................................... 11

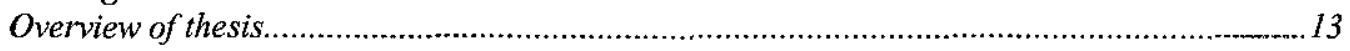

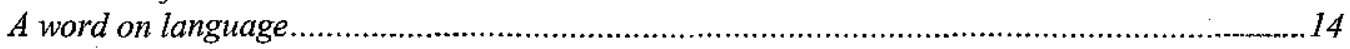

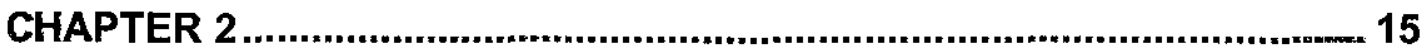

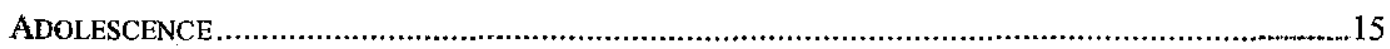

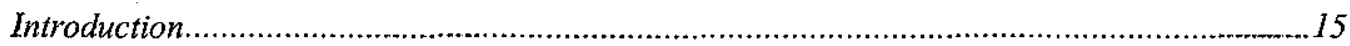

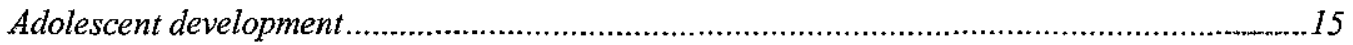

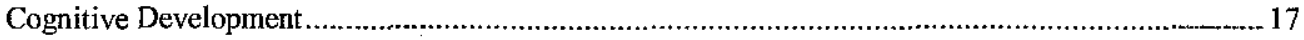

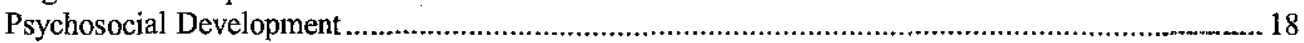

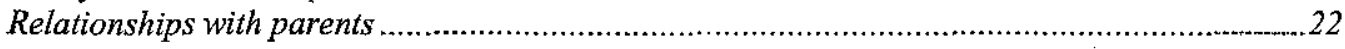

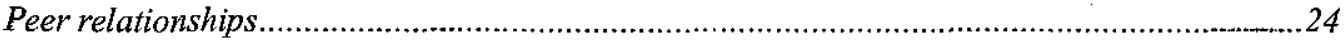

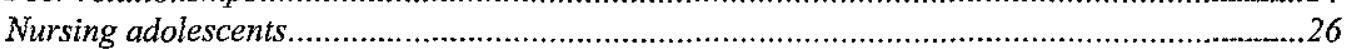

CHAPTER 3

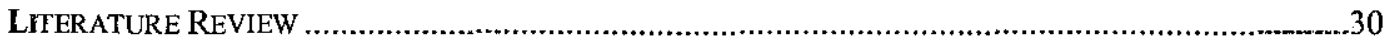

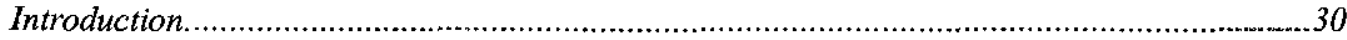

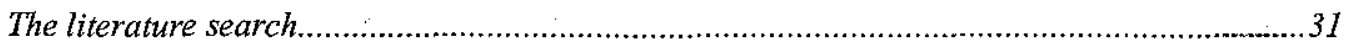

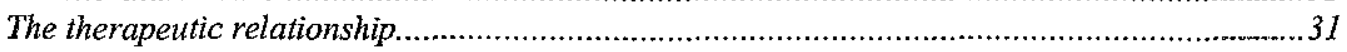

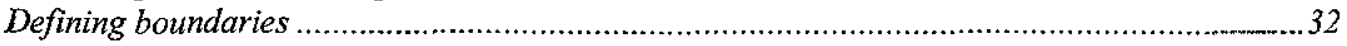

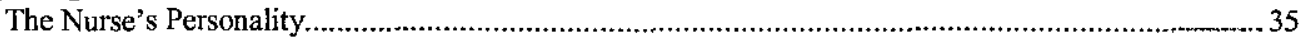

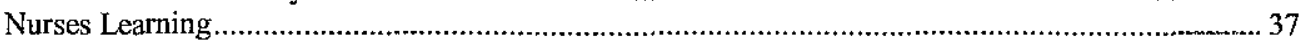

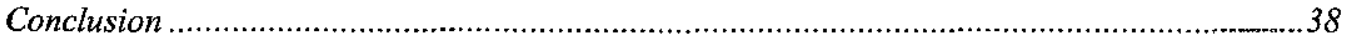

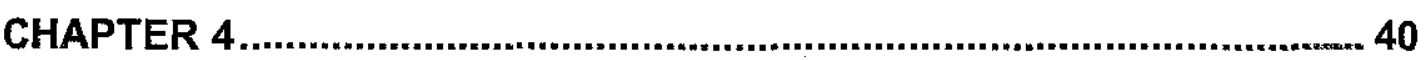

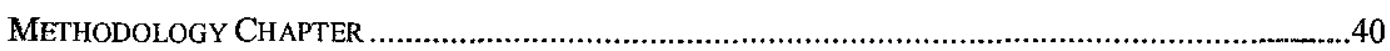

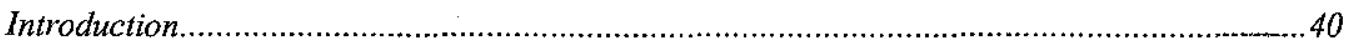

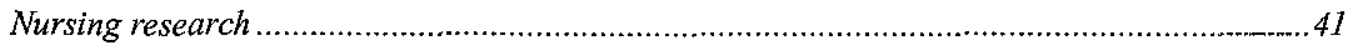

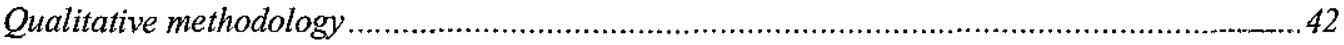

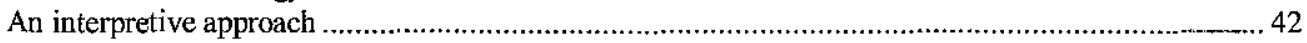

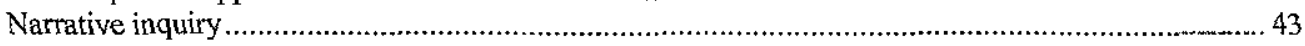

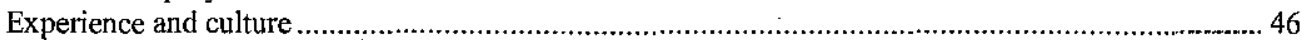

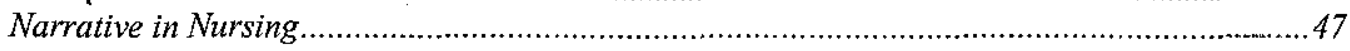

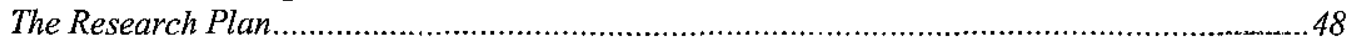

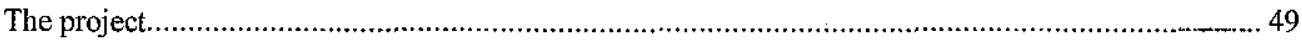

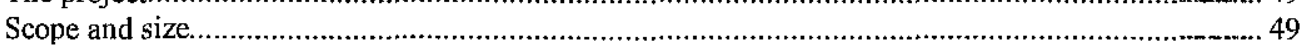

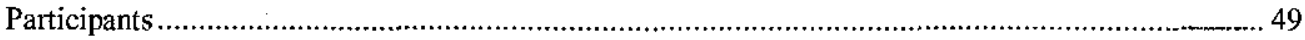

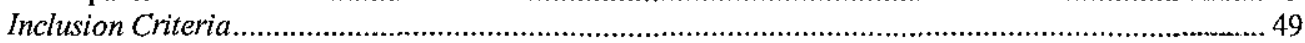

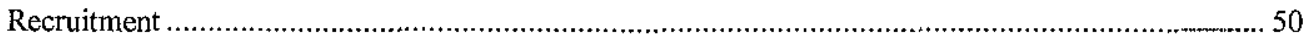

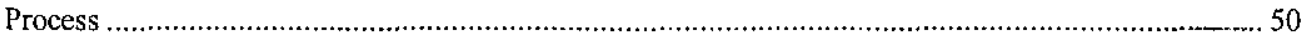

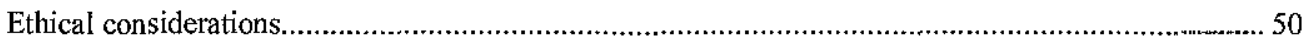

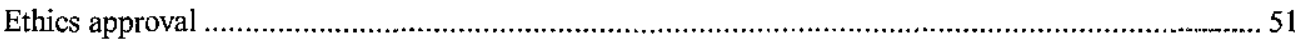

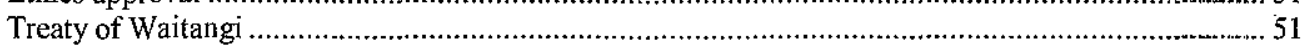




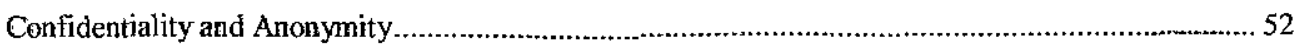

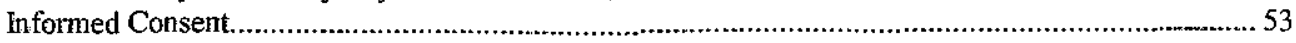

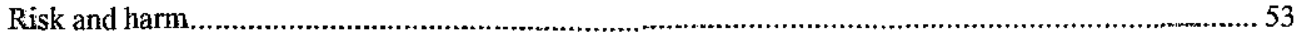

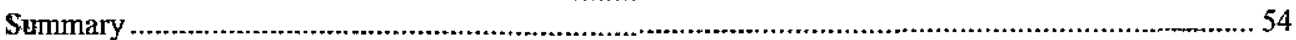

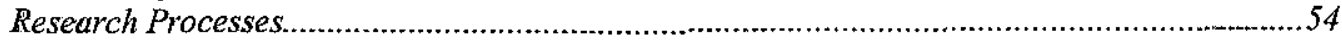

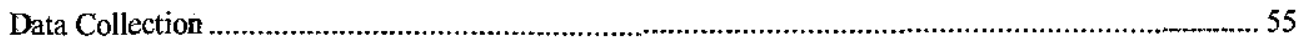

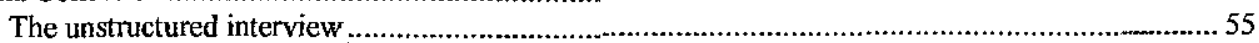

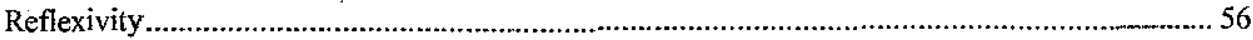

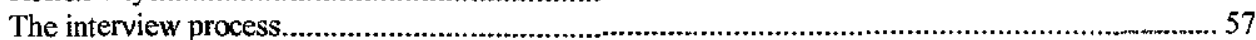

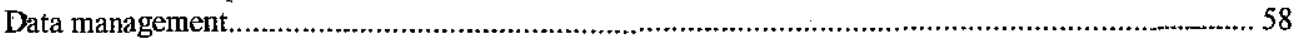

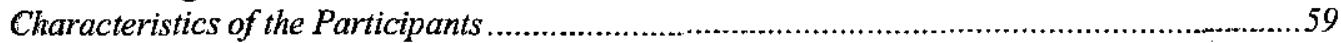

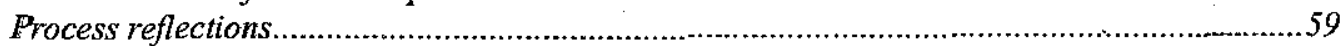

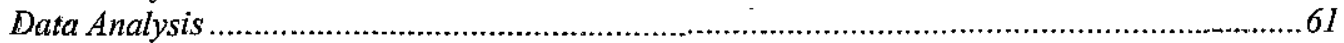

Determining Rigour

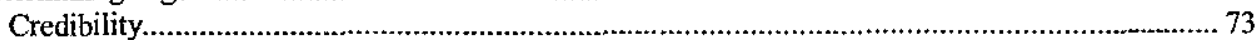

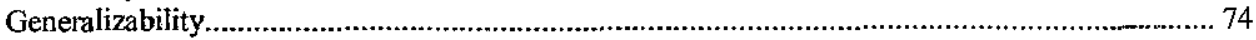

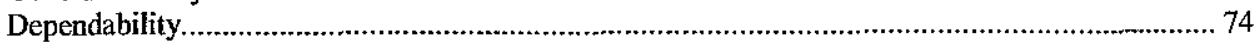

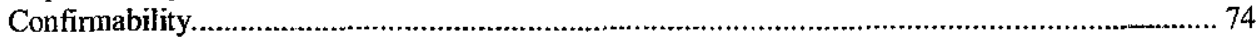

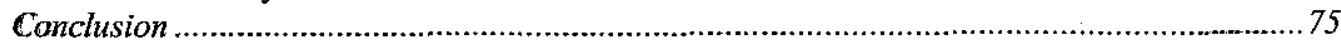

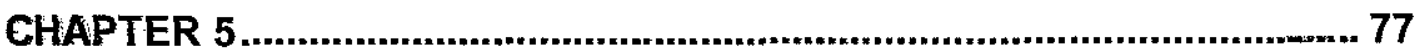

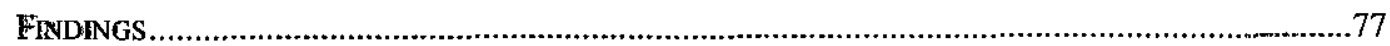

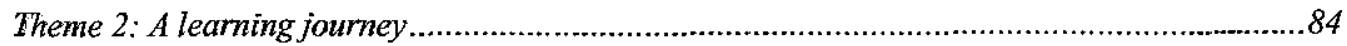

Theme 3: Keeping the nurse and the adolescent safe....................................................8

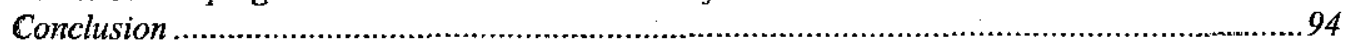

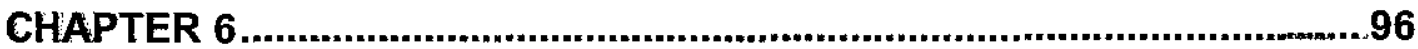

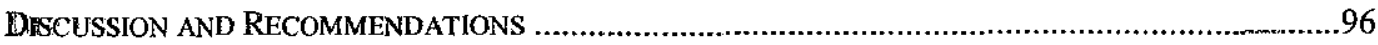

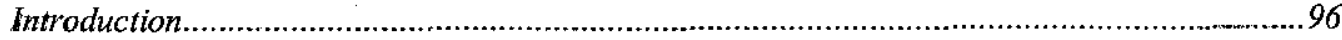

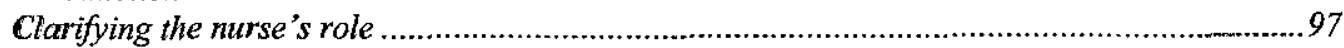

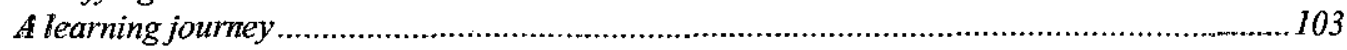

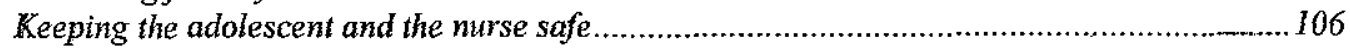

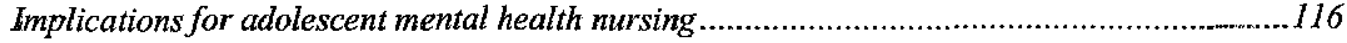

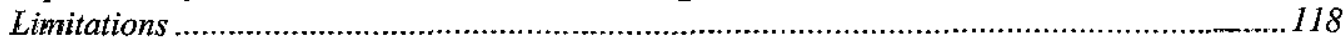

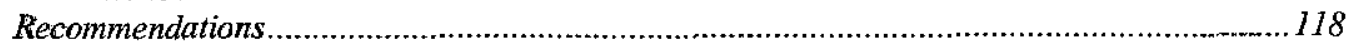

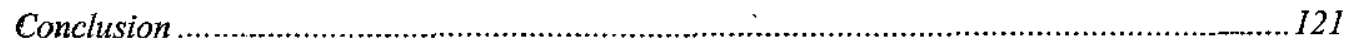

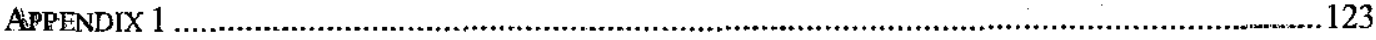

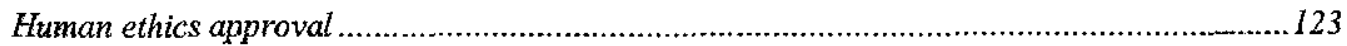

(n) 124

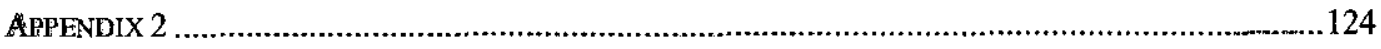

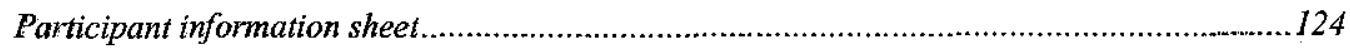

126

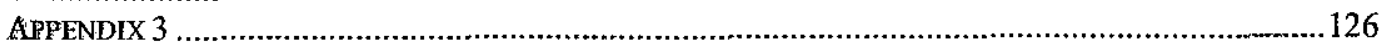

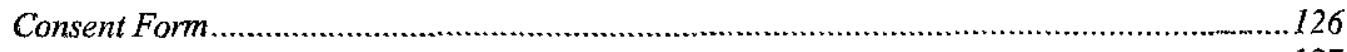

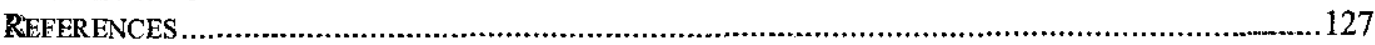




\section{List of Tables}

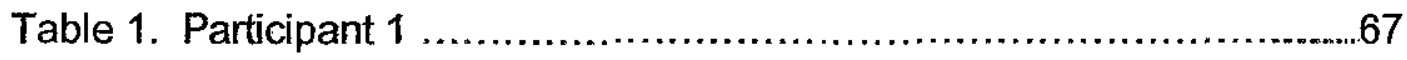

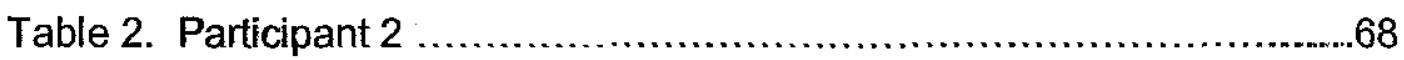

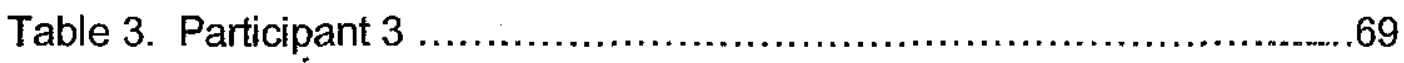

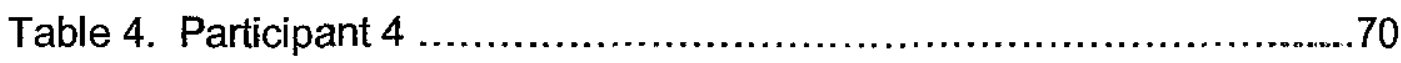




\section{Acknowledgements}

Writing a thesis has been one of the most challenging things I have ever embarked on. It is something that I would never have been able to achieve without the support of many significant people.

I would like to acknowledge the encouragement of Victoria University, Graduate School staff, specifically a special thanks to Thelma Puckey, my supervisor. I am grateful to the New Zealand Nurses Organisation, Nursing Education and Research Fund for financial support during this thesis and Taranaki District Health Board for their supportit and contribution.

Friends and colleagues who played a significant role include: Diane Sadler who has nurtured and inspired my achievements. During mayt trips to Wellington Diane and Peter treated me like a princess. Anne MacDonald, Tony Farrow, Julie Dixon, my Aunty Dawn and my colleagues and friends at Taranaki CAMHS, all of whom provided me with helpful advice, guidance and support.

Thank you to my parents who have encouraged, assisted and privided me with love and attention during testing times and taken time from their busy lives to edit the many drafts of this thesis. Last, but definitely not least, thanks to Tim, a special man who has tolerated living with me throughout the challenges. He has supported, cared and survived living with someone writing a thesis, just! 


\section{CHAPTER 1}

\section{Introduction}

\section{Ko Wai Au?/Who am I?}

Ki ora kotau Katoa/ Hello to all

Ko Taranaki te Maunga/ Taranaki is my mountain

Ko Tawhiti te Awa/ Tawhiti is my river

No Hawera ahau/ Hawera is where I am from

Ko Brian rawa ko Coral aku Tipuna/ Coral and Brian are my Grandparents

Ko Neil rawa ko Beverly aku Matua/ Beverley and Neil my parents

Ko Julia McLean ahau/I am Julia McLean

The use of mihimihi or a personal family introduction at the beginuning of this thesis is significant for me as a New Zealander to position myself, and to provide the reader with an opportunity to understand who I am and where I come from. As an adolescent mental health nurse working in New Zealand, culture and identity play a centrally important role in my life both personally and professionally.

In my experience the sharing of self when working alongside adolescents in a mental health setting is essential in building and maintaining therapeutic relationships. There is very little literature and research on how nurses do this effectively while maintaining therapeutic and professional boundaries with adolescents. This study explores nurses' experiences of assessing, understanding and maintaining therapeutic boundaries, with adolescents in a mental health setting. The specific objectives of the study are:

- To understand through nurses' practice stories, their experience of developing therapeutic relationships with adolescents; 
- To explore how nurses might make sense of therapeutic boundaries in these relationships and how they negotiate these with the adolescent;

- To determine what factors influence nurses' thoughts and ideas around the defining of therapeutic boundaries.

The Australian Nursing and Midwifery Council, Nursing Council of New Zealand and University of Newcastle are currently collaborating to update and develop national guidelines on boundaries of professional practice for nurses and midwives at the time of writing this thesis. This group defines a professional boundary as "the limits that allow a patient/client and nurse or midwife to connect safely and appropriately in a therapeutic relationship based on the patients/clients needs" (The Australian Nursing and Midwifery Council, Nursing Council of New Zealand \& Faculty of Health, University of Newcastle, 2007, p. 2).

The therapeutic relationship is seen as central to the work of mental health nurses by some researchers in the literature (Geanellos, 1997; O'Brien, 1999; Peplau, 1952). Nursing is a planned action with the nurse using his or herself to form and maintain appropriate relationships: with the patient (Carper, 1978). The mental health nurse is often seen as a committed, caring, self-aware individual who works in partnership with her patients (Cutcliffe, 1997). Mental health nursing with adolescents is no different. Adolescents are vulnerable individuals; their personalities are still developing (Elkind, 1998). They require supportive, caring relationships with nurses who have clear objectives of supporting the young person to recovery.

With no current clear guidelines and limited literature on how nurses are managing professional boundaries in these relationships, how does the nurse ensure this care to be safe and appropriate? For the safety of the adolescent and the nurse it is vital the nurse understands her role in managing the professional boundary within this relationship. 
This introductory chapter provides a brief narrative of who am professionally as an adolescent mental health nurse, as this is the basis which led me to research professional boundaries as the centralidea in this thesis. Aspects of culture and identity and the effect my experiences have had on my practice are explored. A brief introduction to adolescent mental health nursing and professional boundaries within this context is provided. The background of adolescent mental health nursing Zealand provides context for meeting the adolescent mental mealth nurses who were the participants in this project. The introduction outlines the journey this thesis takes, and also provides an explanation of the language used.

\section{My narrative}

This section reveals my experiences of adolescent mental health nursing which has led me to research professional boundaries to provide a context for this thesis.

I am a Registered Comprehensive Nurse, educated in Taranaki, New Zealand, in the early 1990s. On completing my training I worked two years in the general health sector before embarking on a working holiday in England. While there, I was employed as a general nurse an adolescent mental health unit. The unit was very well established and nursing was highly valued. During my time there, I was given the opportunity for further training and development.

I completed papers through the English Nursing Board the Psychosocial Care of Children, Adolescents and their Families. In addition there were various clinical in-service training and conferences on adolescent mental health issues. The training and the clinical practice in the units in which I worked had a strong focus on nursing psychodynamically. Peplau (1952, p.xi) describes this as: 
recognising, clarifying, and building an understanding of what happens when a nurse relates herself helpfully to a patient are the important steps in psychodynamic aursing, nursing is helpful when both the patient and the nurse grow as a result of leaming that occurs in the nursing situation.

This relating of self with the patient was something that was very new to me. In my education as a comprehensive nurse very little of the teaching looked at psychodynamic concepts or the role my personality played in nursing. The units in England also had a strong focus on elinical supervision delivered in a way which made me look at the influence: lihad on the adolescent patients.

In addition to clinical supervision nurses were advised to particinate in their own psychotherapy to enable them to work out and process some of the unresolved issues from their personal lives. I participated in psychoanalytical psychotherapy from the Tavistock Clinic in Lamdon. This clinic is a world leader in psychoanalytical psychotherapy and as a 25 year old, I found this quite daunting. However, as I became familiar with this process, I found it incredibly helpful in helping me to process my issues and how l operate. It assisted in separating my issues from those of the unwell young people and their families.

As there was such a strong focus on working psychodynamically, the nurses were very aware of professional boundaries, and the rofe they played in ensuring relationships with the adolescents were therapeutic. Concepts such as 'transference" and 'counter transference' where second language to the nurses in England the majority of where mental health trained. Their three year undergraduate education had little focus on general nursing. The main focus of their education was on mental health; therefore the use of psychodynamic concepts and the influence of their own personalities on patients were familiar to them:

On returning to New Zealand four years later, I was involved in setiting up and running a regional, bicultural, adolescent mental health ingatient

\footnotetext{
'Transference is defined by Casement (1985, p. 7) as "previous experience and related feelings are transferred from the past and are experienced as if they were actually in the present".
} 
service, the Rangatahi unit. As Clinical Nurse Specialist was responsible for the training and development of 16 nurses, few of whom had worked with adolescents in mental health before. This was a challenging task.

The position of Nurse Specialist for the Rangatahi Service was an exciting challenge, one that inspired my thinking about the differences in nursing adolescents in mental health in New Zealand, and the skills required to do this. Although I felt I had a strong sense of identily); (my work at the Tavistock Clinic had helped in contributing to this) I wasn't prepared for the richness the experience of a bicultural service would contribute to my life. Returning home, after spending four years away, signified a return to my culture and my family. However, I was: not prepared for the impact working within a bicultural setting would have enabling me to explore my identity as a New Zealander and a mental health nurse.

I was brought up in a pakeha family where respect for both cultures and family was greatly instilled. I attended government schools where learning things Maori were part of the curriculum. Therefore, I had some understanding of tikanga (how things should be done - things Maori). However, my involvement in the development of the Rangatahi Service enabled me to explore tikanga further.

Mihimihi/introductions enabled me to understand where the people was working with came from, their culture and identity. For Maori Zealand, the significance of whanau/family and where they come from is of importance (Durie, 2003). Individuals are seen first as members of a whanau or Iwi/ tribal group with connections to land, mountain and river of their area which they identify, hence, the inclusion of these in my own thesis introduction. Mihimihi or introductions provide an opporturity for people to find details about where they are from with the aim of breaking down barriers, being open about who they are. It is also an opportunity 
to make connections with a person's extended family or connect to the place where the person was born or brought up.

Whanau or family is a word often used to describe a group of people who descend from the same ancestors and have some biological connection. "In modern times, whanau is also used to describe a group who share not a common heritage but a common mission - a kindergarten vuhanau, a whanau support group, team-mates perhaps". (Durie 2003, p.13). Those in the Rangatahi unit formed a whanau itself, not only the staff that worked there but included the adolescents/rangatahi who were admitted for treatment. As a bicultural service the Rangatahi service aimed to provide treatment to young people and their families experiencing mental illness while at the same time providing an experience of whanau and support they may not have experienced previously. This provided an introduction to the Maori culture and the concept of living and working biculturally.

The unit culture enabled me to think, explore and define who I am as a New Zealander and mental health nurse. However it did raise a lot of questions for me with regards to professional boundaries. It was expected within this milieu that staff share information about themselves, their identity and where they have come from, to assist in role modeling the importance of this for young people. This was a very different way of working from what I experienced in England, as this wasn't the cultural norm. England was a place where it was easier to say I was from New Zealand and a little about my family as it was unlikely anybody \& was working with would have any connections with my family or personal life. in New Zealand this is different; the population is smaller and is common for people to know who your family is or to make connections with your personal life. Because of this, I felt sharing things such as where I grew up may leave me feeling vulnerable and I questioned why it was done. I questioned what was I prepared to share, and who benefited from this? Maintaining professional boundaries within my work was important to me; I needed a way to manage this in a culturally appropriate way. This 
led to my exploration of work experiences through post graduate study and clinical supervision. My goal was to incorporate my views: and experiences of professional boundaries into working biculturally as an adolescent mental health nurse and as a New Zealander. I successfully achieved this goal. During my time at the Rangatahi Unit I managed to find a balance between sharing parts of my life and identity with the adolescents and staff, and ensuring I managed therapeutic boundaries effectively. My role then included role modeling to other staff or whanau members.

A person's life story or personal narrative has an effect on whe person is as a nurse. The need for me to position myself in terms of my professional experience at the beginning of this thesis is core my being a nurse. This project aims to explore nurses' experiences of assessing, understanding and maintaining therapeutic boundaries in an adolescent mental health setting. My passion and interest inspired me to embark on this research and I am committed to ongoing development in this unique area of nursing. Geanellos (1997, p.53) notes the importance of adolescent mental health nurses engaging in research when she says "adolescent mental health nursing continues to borrow theory rather than test and generate its own". I have a passion for adolescent mental health nursing in New Zealand, and believe adolescents experiencing mental health problems deserve the highest quality nursing care available to them.

The first step towards managing the boundaries is engaging with the adolescent in the therapeutic relationship. The following section explores the therapeutic relationship and professional boundaries which is the focus of this thesis.

\section{The therapeutic relationship and professional boundaries}

In this section the definitions of the therapeutic relationship and professional boundaries are discussed using the international literature. 
There are many interpretations of this phenomenon and I include: my understanding and views in the discussion.

The significance of the therapeutic relationship in mental health wursing is well documented (Geanellos, 1997; O'Brien, 1999; Peplau, 1952). However, this relationship is not exclusive to the mental health nurse; but is in fact central to many specialties of nursing (Geanellos, 2002). However, Peplau (1962) states the depth and focus of the nurse patient relationship is more significant in mental health nursing than wor specialties. The nurse uses her skills in engaging the adolescent to work towards change for his benefit. In my experience some of the nursing skills applied to establish a rapport with adolescents experiencing mental health problems include; a good sense of humour and play, ewergy, enthusiasm, creativity and sense of fun. Openness, honesty and respect are other helpful skills in engaging the adolescent in a therageutic relationship.

Professional boundaries within the relationship play a big part ensuring the relationship remains therapeutic. de Raeve (1998, p.136) states "engagement may be important but so also is the maintenance of a degree of distance and objectivity, which is essential for both the nurse and the patient in order for nursing to occur". Barnsteiner and GillisDonovan (1990, p.226) define a boundary as "where self leaves offi and the other person begins. Individuals' boundaries define their unique individuality". When nursing patients in mental health the closerress of the therapeutic relationship makes it essential the nurse defines; the therapeutic boundary. If the nurse cannot do this, the nurse and the patient are at risk (Sheets, 1999).

When considering boundaries within the therapeutic relationship the nurse asks herself about the power differential within the relationship, professional distance, friend versus friendliness, reciprocity in relationships and the amount the nurse self discloses (Curtis \& Hodge, 1994). Most authors refer to the need for the nurse to ascertain the 
purpose of the interaction or relationship, as interactions need have clear planned intention. Is the interaction or the relationship in best interests of the patients? (Curtis \& Hodge, 1994; Sheets, 1999; Barnsteiner \& Gillis-Donovan, 1990). de Raeve (1998) also emphasises the importance of both parties (the nurse and the patient) being sure to respect each other's privacy and not intrude on issues within each other's life they might not be ready to disclose.

It is the nurse's responsibility when working with young people experiencing mental illness, to have an awareness of her role the therapeutic relationship and the management of professional boundaries. Scanlon (2006, p.323) suggests

psychiatric nurses are professional people; however, they are people, with the same expressions of emotions and feelings as the people they are treating. This is seffected in confusion in relation to the degree of personal experiences they should or strauld not express to the patients.

When I am working with a young person and the family, I believer it is important for the young person to know a little about who I am, I come from and my work experiences. This assists in the engagement process. The importance of being sincere and genuine my interactions with young people and their families is significant. Alongside this I need to be clear about my role, and open and honest the young person. The needs of the adolescent should always be at the forefront. To engage the adolescent in the therapeutic relationship the nurse often has to give or share something of herself. Within the mursepatient relationship the importance of being aware of how much give to maintain a professional boundary is an ongoing dilemma. OBrien (1999) acknowledges the double blind contradiction between maintaining professionalism and ensuring the role isn't overtly professional as this would conflict with the nurse's ability to ensure a therapeutic relationship.

O'Brien (1999, p.158) suggests the need for the nurse to remain "wuman and accessible to clients". The nurse needs to be accessible or avaifable psychologically to the adolescent to have an interaction with him. She needs to be attuned the adolescent's needs at the time of interaction. 
This means the nurse isn't thinking about her own issues or other things when she is having an interaction with the adolescent. When the kurse is able to do this, she is aware of her role within the relationship and conscious of what is be shared. The nurse needs to be aware of the reasons for sharing information about herself or about other young people she has worked with. This sharing should benefit the adolescent. In the work I do with adolescents I am astutely aware of what I share and question myself about who is benefiting from this sharing. If sharing information for my benefit, to off load or treat the relationship as I would a friend $_{x}$ I am not respecting my position within the relationship.

I am also conscious of the need to give some of myself to the adolescent but would do so only about issues specific to me if the issues had already resolved and had I time to reflect in terms of the effect they/ had on my life. This ensures that I am not using the relationship to solves my personal issues. In psychotherapy Rogers (1980, p.148) acknowlediges "the more psychologically mature and integrated the therapist is; the more helpful is the relationship that he or she provides. This puts a heavy demand on the therapist as a person". This is equally twue for mental health nurses especially when working with adolescents who are actively seeking role models to base their experiences on, it is beneficial for the adolescent when the nurse has a clear understanding about herself. The situations the nurse may relate about herself should be relevant to the adolescent's current situation. This ensures the rurse is working to meet the needs of the adolescent by this sharing.

When I am asked about my private life by adolescents I am conscious and careful what I share. I often explain explicitly to the adolescent my role as a nurse and the importance of my private life being separate: from my work life. I explain that I will at times share stories of my life 萿I feel they are relevant. It is important for the young person to know that nurses are human too, and have periods in our lives that are difficult, or that events also cause us stress and we all need to find ways. of managing this. Gastmans (1998, p.5) acknowledges this saying, "the 
nurse and the patient are conceived as human persons, each with their own fields of experience and perception, constituted by thoughts, feelings, desires, assumptions, expectations and activities". Through this sharing the nurse may role model coping strategies or managing difficult situations.

Nurses can be faced with managing difficult situations everyday. Thave found living and working in my home province a challenge; in particular reflecting on how I manage the potential conflict of living in a small community. This requires that I ensure my boundaries are professional and therapeutic. Recognition of these dimensions in my practice mean that my current work in my home province for the Taranaki District Health Board, as a child and adolescent mental health nurse, is always dynamic. The skills I gained in working biculturally at the Rangatahi Service and nursing psychodynamically in England remain relevant in my work with Taranaki families. Reflecting on these experiences, I realise the management of therapeutic boundaries in relationships with adolescents is complex. Nurses bring previous experiences from education and often extensive nursing experiences. When exploring nurses' experiences of assessing, understanding and maintaining therapeutic boundaries with adolescents it is important to know something about the nurse's background. This includes their mursing education, professional development and leaming opportunities.

\section{Nursing education in New Zealand}

This section looks at undergraduate and post graduate education available to nurses who may be working in child and adolescent mental health services in New Zealand. It is important to consider the nurses' education experiences as this may provide a context for thinking about nurses' experiences of maintaining professional boundaries and education around this. It also positions nurses currently employed to work with adolescents experiencing acute mental illness who responded as participants in the project. 
Undergraduate education in New Zealand consists of a three-year Comprehensive Degree programme covering a broad range of nursing knowledge and skills with a strong focus on general nursing. Peters (2003) found the mental health component of these undergraduate nursing programmes minimal, with some courses having as little as two weeks general mental health theory and 160 hours of clinical experience in a mental health setting during the three year programme. Some of these programmes had less than an hour focused on child and adolescent mental health issues. Prebble (2001) found in her study a number of undergraduate nursing programmes do have a strong focus on mental health. However, the quality and visibility of this mental health component was dependent on the personalities and qualities of only one or two tutors on the staff. This remains a concern for the nursing specialty. The mental health content of the programmes in 2003: was focused on diagnosis, risk management and cultural issues; anything related to nursing psychodynamically is not discussed (Peters 2003).

Some polytechnic education providers have postgraduate programmes for comprehensively trained nurses to work in mental health. These Nursing Entry to Specialist Practice Programmes are funded by the Clinical Training Agency (CTA), a Ministry of Health (MoH) fund. Some of these one year programmes offer a child and youth stream. This provides the small number of nurses on the programme with the opportunity to gain experience and knowledge in child and adolescent mental health nursing and wider skills and knowledge related to mental health nursing in general. The Werry Centre (2004) reports in 1998 the CTA requested a proposal to support professional development and to up-skill the workforce specifically in child and adolescent mental health. Various universities in the country set up specific multidisciplinary child and adolescent mental health post graduate courses, however, due to insufficient numbers, some were closed. The $\mathrm{MoH}$ recognised the country's shortages and attempted to address funding to up-skill this workforce. However, most of the content of these courses was based on 
generic community care for adolescents with mental health problews: and none were designed solely for nurses. Geanellos (1999) found the greatest numbers of mental health care providers are nurses, however, in Australia (and New Zealand) there are no courses available specifically in adolescent mental health nursing. It could be argued that the small population in New Zealand means that generic postgraduate courses in child and adolescent mental health should be sufficient. However, this undervalues the significant role nurses play in addiescent mental health. It may also limit the amount of education that is available for nurses on nursing specific issues, such as psychodynamic nursing principles which would benefit the nurses in managing professional boundaries effectively.

Although these courses are available, nurses are continuing be employed into child and adolescent mental health services withaut any postgraduate education in this area. In New Zealand there is a problem in general of recruiting nurses into the mental health field (Walsh, 2002). Because of this problem nurses without any post graduate qualifications are employed.

Without a specialist adolescent mental health nursing programme min $\mathbb{N e W}$ Zealand can nurses identify themselves as adolescent mental health nurses? Geanellos (1997, p.57) notes the same problem exists in Australia; with no specialist adolescent mental health rursing qualifications she questions "how will nurses working in this specialty be differentiated"? These are all areas that need to be addressed are beyond the scope of this thesis. This overview of adolescent mental health nursing education in New Zealand provides a context the adolescent mental health nurses involved in this research project.

\section{Overview of thesis}

The aim of this thesis is to contribute to nursing knowledge of murses' experiences of professional boundaries in adolescent mental heath in 
New Zealand. Using a narrative research methodology this study aims to elicit nurses' experiences of assessing, understanding and working with therapeutic boundaries when working with adolescents in a mental health setting. I discuss some issues pertinent to working; with adolescents in mental health. This includes a chapter on adolescent development to provide an understanding of why nurses need to: be aware of adolescent development issues when working in this area. The literature review discusses relevant available literature related to therapeutic relationships and professional boundaries when working in this area. The theoretical underpinnings of a narrative methodology in nursing research is discussed, followed by an in-depth explanation of the research design. The findings of the research are explained utilising the nurse participants' practice stories. The findings are discussed! and compared with relevant literature. The discussion chapter cancludes with a section outlining the limitations and possible implications: for nursing practice in this complex area. It is hoped that this researchi will benefit not only nurses working in this field but also nurses working in adult mental health inpatient services, and paediatric wards, where many adolescents requiring inpatient provision are currently nursed.

\section{A word on language}

When I have used the term 'adolescent mental heath nurse' this refers to nurses currently employed in mental health positions working specifically with adolescents. The term 'nurses' refers to nurses registered in New Zealand. 'Paediatric nurses' refers to nurses working in a children's ward who are involved in nursing adolescents. The term adolescent refers to young people aged between 12-19 years. Adolescent is the term mainly used however, young person, teenager and/or youth may be used interchangeably. For ease of reading, nurses have been referred to as she and adolescents as he throughout this report. The word 'story' refers specifically to individual practice stories narrated by the nurse participants, while 'narration' or 'narrative' refers to the whole experience of the interview with the nurse. 


\section{CHAPTER 2}

\section{Adolescence}

\section{Introduction}

This chapter looks at the developmental period of adolescence. Adolescence is a significant developmental stage. There are hallmarks related to adolescent development that need to be considered when discussing therapeutic relationships and the use of therapeutic boundaries in mental health nursing. This chapter addresses some of the issues related to working with adolescents aged between $12-19$ years, which are essential for nurses working with this age growp to understand. Key developmental theorists such as Erikson (1963) and Piaget and Inhelder (1969) provide a theoretical framework to inform both the cognitive and psychosocial issues of this developmental period. Erikson's (1963) theory on identity formation in adolescence is inturential when exploring this developmental stage. That is not to say that other developmental theorists do not have valuable contributions to this stage in the human life-span. Theorists utilised to discuss this developmental phase further include Hamburg (1998) and Elkind (1984). The stages of adolescence are discussed separately as younger, middle and older adolescents as the developmental issues vary significantly from younger to older adolescent. The relationship adolescents have with parents and peer groups is explored, alongside behaviours such as risk taking and establishing a social identity. The role of the mental health nurse when working with adolescents is examined.

\section{Adolescent development}

Adolescent development is commonly referred to as being particularly complex. This section identifies some of the issues for adolescents 
related to their psychosocial and cognitive developmental tasks that contribute to this complex development journey.

Age 12-19 years is the current age bracket identified for access to Adolescent Mental Health Services in New Zealand. There is discussion around the lengthening of the age bracket the term 'adolescence" spans, with varying opinions on the actual age range it now incorporates. Hamburg (1998) found adolescence is from age 11-21 years due to developmental needs of youth often not being met until the earfir. 20 's. Young people in the developed world are currently more inclined to attend university and/or stay at home longer; therefore, are often financially reliant on their parents for longer periods of time, hence delaying the developmental tasks of independence and lengthening of the adolescent period (Hamburg, 1998). This may be a consideration: for adolescent mental health services when working with the older adolescent who is still very reliant on his parents.

Adolescence is a very significant developmental stage in terms of life transition (Hamburg 1998). The adolescent is moving from being a child into adulthood which brings many challenges. They are finding their way, searching for their identity and recognition of who they are from their peers and parents. It is a time in a young person's life whew he is no longer a child and has not yet moved into adulthood (Hamburg 1998). Geanellos (1997, p.21) acknowledges the complexity of this stage when stating "adolescents vacillate between the thoughts, feelings and behaviours of a child and those of an adult". The young person will eften expect to be treated like an adult which brings many new challenges. At times they want the freedom to go to parties and stay out late yet miss school by sleeping in the next day (Frankel, 1998).

The length of the developmental stage and diverse experiences encountered by adolescents brings many challenges for the adolescent himself, parents and nurses working with him. The need for surses working alongside adolescents experiencing mental illness to have: an 
understanding of adolescent development cannot be overestimated. Adolescent neurological pathways and thinking patterns are: still developing (Piaget \& Inhelder, 1969). There are specific differences the nurse needs to recognise. The following section uses research to affer a definition of the developmental stage for adolescents in cognitive development which is useful in practice conversations.

\section{Cognitive Development}

This section on cognitive development draws on the works of Piaget who developed the notion of formal operational thought and the works of Elkind (1984), who expands on this and the notion of egocentrism in adolescent development.

Adolescence is the time in life when cognitive ability develops from concrete operational thought to formal operational thought (Piaget \& Inhelder, 1969). Here the adolescent has the ability to hold on to and combine multiple realities and concepts, and is able to engage in abstract thinking.

Formal operational thought enables the adolescent to reason with things through thinking about the thoughts occurring inside his head. They can reason with their own thinking but also think about thoughts of others. Elkind (1998, p.36) states

Nonetheless they tend to make a characteristic error when they engage in this kind of thinking. Because they are going through such a radical transformation in theight, weight, physical appearance, emotional expansion, and intellectual transformation they tend to be self-centred. Accordingly, when they think about other people's thinking, they automatically assume that others are thinking about what they are thinking about, namely, themselves

This also gives rise to self consciousness and at times can exacerbate adolescents' worries about how others may view them. Frankel (1998, p. 125) states adolescents do not yet have the ability "to apply adult fitters that lessen the glare and intensity of the lived world". That is, how they are perceived by others may have much more of an effect on the developing adolescent. 
Elkind $(1984$, p. 36) conceptualised this stage of development as the "personal fable", believing that adolescents have "an inflated opinion of their own importance. They begin to feel they are special and urique". This adds to the already recognised concept of egocentrism which is often related to adolescents. The adolescent experiences an "imaginary audience" (Elkind, p. 36) where he imagines and believes that othersisee him as someone special, bad things will not happen to him only to others. The adolescent sees himself as being invincible. This leads to engaging in behaviours which include "social experimentations limit testing and risk taking" (Elkind 1998, p. 36). This can be challenging for parents and caregivers concerned with the adolescent's vulnerability at this stage of development.

Cognitive development is an important aspect of adolescence. The adolescent who has achieved formal operational thought and is experimenting socially can be very vulnerable. Having parents: and nurses who understand this stage of development is helpful as: this ensures they are thoughtful about and can process some af the behaviour and interactions with the adolescent. Without this understanding they may think the adolescent is being selfish ar spoilt and treat him as such. As well as cognitive development issures the adolescent is faced with psychosocial issues related to their pinysical development, which can bring challenges to the adolescent and those caring for him.

\section{Psychosocial Development}

The discussion on psychosocial development draws on the works Erikson (1968) and Hamburg (1998).

Erik Erikson (1963) is one of the most influential developmental theorists on the formation of the adolescent identity. According to him there: are eight psychosocial stages of development in the human life span, ranging from birth to late adulthood or the elderly. He describes 
adolescents as experiencing the developmental stage "identity verses role confusion" (p. 261). Adolescents are striving to achieve a sense of identity both amongst their peers and within their family and community. Erikson (1968, p. 165) describes it as

optimal sense of identity .. is experienced merely as a sense of psychological wellbeing. Its most obvious concomitants are a feeling of being at home in one's body, a sense of 'knowing where one is going', and an inner assuredness of anticipated recognition from those who count

Those are often adults (including parents and teachers) and the young person's peer group (Elkind, 1998; Hamburg, 1998; Noller, 2005). When an adolescent is unable to achieve this stage he may be left feeling confused about his role and place within his peer group, family and community and the transition into adulthood is more difficult (Exikson, 1968). These occurrences can contribute to a young person being more at risk of experiencing mental illness (Nollier, 2005).

Hamburg (1998) identifies three distinct phases of adalescent development; early, middle and late adolescence. He considers that a failure to recognise these phases can lead to undue pressure being put on younger adolescents.

The period of early adolescence can be a particularly challenging time as it is usually the time when the adolescent experiences most rapid changes of brain maturation, body growth, body changes and puberty (Erikson, 1963; Hamburg,1998). The timing of the adolescent's biological changes of puberty are significant. Body image is an important aspect of a young person's identity, therefore timing, rate and outcame of these impact on the developing adolescent (Hamburg). In my clinical experience any vastly different rate of change to an adolescent's peers, can have a marked influence on the adolescent's self esteem.

Early adolescents are often pushing for independence (Hamburg, 1998, p. 36). However, young people still require much support and guidance from parents and other adults throughout this phase as they are "moving 
in and erratically in and out of the world of childhood". This struggle for independence is discussed later in this chapter when looking at the young person's relationship with his parents.

Middle adolescence is described as a much less stressful period than early adolescence as there are no significant physical changes. The physical changes occurred have been consolidated, and the young person has a clearer sense of self physically (Hamburg 1998), and there is limited pressure on the adolescent to be thinking about career options and independence.

Hamburg $(1998$, p.36) considers that "late adolescents are at the threshold of the adult world, trying to establish themselves: as independent persons". This is the phase in which a sense of individuality and identity become the focus as the young person is making decisions about an occupation path and moving towards living independently. This can be a challenging time for the adolescent to be faced with having to make so many decisions about his future at once.

Throughout all three phases of adolescent development identity formation is significant. The development of identity is a major task of adolescence (Croghan, Griffin, Hunter \& Pheenix 2006; Elkindi. 1998; Erikson, 1968; Hamburg, 1998; Hendry \& Kloep, 2005; Sharland 2006). The adolescent is attempting to discover who he is, what he believes in, and is forming opinions. Elkind (1998, p.35) found "the construct of a sense of personal identity takes time and effort. In addition; it presupposes the formal operations of intelligence, since these permit the coordination and integration of the many different, sometimes oppesing, strands of the individual's selfhood".

How adolescents portray themselves to others in how they speak look, dress and their overall style is significant of the development of as social identity for the young person (Erikson, 1963; de Klerk, 2005; Hendry \& Kloep, 2005; Frankel, 1998). How the adolescent is viewed in his wider 
social circle including parents and other adults, has a significant mpact on how the young person views himself (Erikson, 1963). Whem the adolescent is able to utilise the connections with these significant people in his life to experiment with his behaviour and interactions, his sense of self and social identity is able to develop effectively. The importance of learning new communication skills, and having the abdity to communicate with people of all ages, is necessary in the progression to adulthood. The period of adolescence when young people learn to rehearse these skills and competencies (Hendry \& Kloep, 2005), If young people have a wide range of communication skills, this hefps their feelings of acceptance of their social identity (Hendry \& Kloep, 2005). These social skills are often practiced with significant others such as parents, grandparents and siblings.

The search for an identity, and the need to fit in, involves the adolescent engaging in risk-taking behaviour, which can challenge the relationship with his parents. Sharland (2006, p.260) found "we must recognise that risk taking is integrally bound up with the development of young people's identities. We must start by recognizing risk taking as a routine even desirable component of young people's lives and development". This risk taking behaviour (as long as it's not life threatening or law breaking) may assist the adolescent in developing social skills and knowledge around some of life's adversities and ability to cope in the future. It commonly involves breaking adult rules including the use of swearing or slang particularly among the adolescent's peers (de Klerk, 2005).

Hamburg (1998, p.46) identifies factors associated with adolescents completing this developmental stage with a clear sense of identity, confidence, a capacity for healthy relationships and to be successful adults. These factors include the adolescent having access to "caring adults" and peers who have a helpful influence. Adolescents need clear messages about adult role expectations and adults who act as respected role models, a supportive group including family, peers, school and community who work together to achieve goals. They also need 
apportunities to achieve success, and this to be rewarded meaningfully, opportunities for work that promotes "self-respect and self-sufficiency" (Hamburg, 1998, p.46).

The adolescent's search for a secure identity is significant in his psychosocial development. The importance of physical development, helpful peer relationships and supportive adults, all contribute the adolescent achieving this developmental stage effectively.

\section{Relationships with parents}

The challenges parents may face in their relationship with an adolescent are discussed next. Nurses working with adolescents trying to establish professional boundaries need to have an awareness of these challenges as there maybe issues related to this that arise in the adolescent-nurse relationship.

The family situation, and relationship the adolescent has with his parents, play a significant role in the adolescent having a smooth transition into adulthood (Noller, 2005). Worden (1991, p.4) suggests parents of the adolescent often have to act as a "buoy; the adolescent may move away from or toward it, but the buoy remains consistert and predictable". My experience indicates this is even more difficult with parental separations and blended and single parent families. Noller (2005) emphasises that adolescents need to have many 'protective factors' within their family unit. These include a cohesive, supportive, and positive relationship with both parents and other family members. Noller also states when adolescents have an experience of poor family functioning and weak bonds with their parents, there are risk factors in psychosocial functioning. This puts adolescents at risk for mental health issues. Parents and parental figures therefore need to be available to the adolescent, without these the young person may struggle to find appropriate role models and security figures to bounce back and forth from. Adolescents who are not able to experience a consistent and predictable relationship with their parents often seek other connections 
with adults in their environment. These include teachers, employers, friends' parents, extended family members, social workers and nurses.

It can be a struggle for parents to know how much support or freedam to allow their adolescent. The literature emphasises the importance of parents being consistent yet flexible (Hamburg, 1998; Williams \& Thurlow, 2005; Worden, 1991). Whilst the adolescent is attempting to gain autonomy at this stage, they still need their parents support to achieve this, even though adolescents may be rejecting it at times.

The adolescent needs to know rules and boundaries exist regarding their behaviour. Within these rules and boundaries there needs to be room to experiment and negotiate. Noller $(2005$, p.216) discusses the significance of positive communication within families as a factor in enabling this flexibility to occur. She states "communication should also be clear, rather than vague or ambiguous, and family members should feel free to express their true feelings and attitudes and to talk about the relationships between family members". This provides an environment where communication is explicit and the adolescent can discuss his views openly.

Respect within family relationships is also considered important with young people in this developmental stage. Although adolescents may not always behave respectfully to others, Williams and Thurlow (2005, p.44) state "there is often a tendency for adults to view adolescents as unskilled communicators" which is not necessarily true. Geanellos (1997) also found adolescents are often viewed as 'anti establishment', and difficult to get along with. Adolescents need to be shown respect (Drury, 2005) and given opportunities to discuss their views in a way that is appropriately role modeled by parents and other adults. Noller 2005 , p.212) outlines the importance of positive communication within families "to show respect and regard for other family members, even during disagreements, so that family members can share their ideas without fear of being put down or belittled". 
The need for adolescents to have supportive, open and respectful relationships with their parents helps adolescents feel secure. Parents can be role models for adolescents in their relationships providing positive experiences in their interactions with others. The other major influence in the life of an adolescent is his relationship with his peers:and this is discussed next.

\section{Peer relationships}

Young people of the same or similar age bracket make up a peer group. This section discusses the importance of peer relationships: for adolescents. It explores the effect positive and negative peer relationships have on the developing adolescent.

While teenagers are developing independence and autonomy from parents they are also creating supportive and satisfying relationships with peers who have less parent or adult oversight (Williams \& Thurlow, 2005). Adolescents rely on their relationships with their peers for multiple reasons; a sense of belonging, feel acceptance, to act as role models, mentors, and confidents; to compete against and for support (Hamburg, 1998). Frankel (1998, p.118) states

adolescents engage with one another in a direct and uncensored manner. What is so moving in these conversations is the level of vulnerability that is allowed in as well as the willingness to share complicated feelings with each other. It is an offering up of the self, in dramatic and playful ways that is not often repeated in adult friendships.

The peer groups chosen by the adolescent can have a posidive: or negative effect on his development. Risk taking behaviour often accurs with peers (Hamburg, 1998). Adolescents can experience the dilesma of wanting to partake and listen to their friends, versus listening to the rules and limits set by parents (Worden, 1991). Their awareness of other adolescents' experience of risk taking behaviours can affect their own ability to cope in these situations (Elkind, 1998). The adolescent learns new coping strategies from one another through hearing one anather's' 
experiences. Young people take their friendships very seriously: these relationships can be a significant opportunity for learning about himself. Positive relationships with other adolescents can have a marked effect on a young person's self esteem and sense of self worth (Hamburg, 1998).

The developmental period of adolescence is distinct and challenging. Adolescents are coping with changes in their physical body, their cognitions from concrete operational thought, to formal operational thought. This gives them a new insight into the world. They are searching for a sense of identity: Adolescents experiment with adult relationships and with their peers. Other psychosocial challenges include a move away from their parents and caregivers towards having more autonomy and independence. However their need for support from parents and other adults remains. This brief summary of adolescent development has looked at the development of 'well' adolescents. Unfortunately not all adolescents are well, with some experiencing abuse, bullying and other occurrences that can lead to mental health issues.

Geanellos (1997, p. 21) emphasises the need for health services to recognise normal adolescent behaviour versus abnormal behaviour: "For instance, normal variations of mood versus marked, sustained mood swings; egocentricity versus narcissism; challenging authority versus abusive behaviour". The clinical ability to discern normal from abnarmal behaviour is critical in adolescent mental health nursing and underpins the assessment conversations and the nurse's ability to ascertain the quality or the acute nature of the behaviour. The difficulty is, adolescence is a time of turmoil which is normal but it can obscure the need for treatment of emotional disorders in adolescents who need assessment by mental health professionals (Hamburg, 1998). Adolescents require specialist care by nurses skilled and knowledgeable in providing care for acutely unwell adolescents experiencing mental 
health problems. There are many challenges nurses face when working with these young people.

The following section explores some of the issues for wursing adolescents whose development has not progressed as smoothly as hoped and therefore are experiencing mental illness.

\section{Nursing adolescents}

Nurses are the majority of the workforce in inpatient mental health services. Overall in child and adolescent mental health services (CAMHS), nurses are one of the three largest occupational groups alongside Psychologists and Social Workers (Ramage, et al., 2005). This section briefly outlines the role of the mental health nurse andl some challenges nurses working with adolescents in mental health may face. These include challenges when nurses are not aware of an adolescent's developmental stage and the nurse's ability to assess, understand and work with boundaries within the therapeutic relationship with the ad'olescent.

Nurses use themselves to connect with and form therapeutic relationships with adolescents. This can be done in many differentways depending on the personality and previous experiences of the murse. The therapeutic relationship is seen as central to the work of mental health nurses by some researchers in the literature (Geanellos, 1997; O'Brien, 1999; Peplau, 1952). Within the therapeutic relationstip the mental health nurse works alongside her patients to empower them; she regards them holistically (Cutcliffe, 1997) with the aim of supporting; the client to accomplish his goals. Nursing is a planned action. The nurse uses herself to form and maintain appropriate relationships the patient (Carper, 1978). Mental health nursing with adolescents is: no different. Adolescents require supportive, caring relationships; with nurses who have clear objectives of supporting the young person to recovery. 
Murray and Wright (2006, p.161) describe "the first step in establishing a meaningful relationship with adolescents is connecting with themi and engaging them in the relationship. This requires genuine interest on the part of the mental health professionals." Their research found a non judgmental approach allows the adolescent to express himself effectively and that "adolescents are usually very astute at identifying a lack of sincerity or acceptance" (p.161). An adolescent's feelings of acceptance can lead to an improved sense of wellbeing (Kimberly, 2002). Teenagers often view nursing staff as friends (Geanellos, 1997; Kimberly). However, as Kimberly (p.88) states although "the friendship might not extend beyond the hospital or fit into the adult's definition of friendship, it is nevertheless a vital stabilizing force for isolated and frightened adolescents". The relationship or friendship can portray to the adolescent that he is accepted as a person, which can aid the adolescent's journey towards wellness. However, this relationship is offen difficult to negotiate and nurses often struggle with getting a balance between forming bonds which are too close with the adolescents or becoming like authoritarian parents (Kimberly).

In my clinical experience when nurses form close bonds with the adolescent, he will often idealize the nurse; an adult whom the young person feels listens and understands him. The adolescent is developmentally separating his identity from that of his parents, which at times can create conflict with the adolescent and his parents. The murse, who is in the helping profession, may not be aware of this dynamic. When this occurs there is a risk of the nurse allowing her professional boundaries to blur. The adolescent is projecting a need to be taken care of and understood by an adult, this could create a feeling in the nurse that she needs to do more than her role allows. The nurse needs to be aware of this potential dynamic and keep her professional boundaries and role clear. The therapeutic relationship with an adolescent is complex; therefore it is vital the nurse has an understanding of where the adolescent is in terms of developmental needs in ensuring this relationship remains therapeutic. 
When nurses have an awareness of adolescent development their ability to make sense of some of the behaviour is enhanced. Adolescents are at a stage where they are exploring their identity and in transition from childhood to adulthood. Geanellos (1998, p.241) found "adolescents vacillate between the thoughts, feelings and behaviours of a child and those of an adult". They often display very challenging, rejecting behaviour towards both their family and at times the nursing staff (Kimberly, 2002). This can be described as similar to behaviour of a patient with borderline personality disorder ${ }^{2}$ Krawitz and Watson (1999, p. 17) state "having traits of this disorder is probably a normal feature of adolescence." When adolescents behave in a challenging way, or clinicians believe a person has these borderline traits or diagnosis, a derogatory attitude towards the patient's right to treatment is shown (Kimberly; Krawitz \& Watson) which has a negative influence on: the already troubled adolescent. Kimberly (p.84) found "such behaviour might not be recognised immediately as "the outward manifestation of coping". This behaviour may appear inappropriate to staff those knowledge of adolescent developmental issues is limited. More problematic is when the young person is in late adolescence as: the expectation for the adolescent to behave like an adult is higher.

When nurses in the field are competent they understand the parameters and diverse expressions of adolescent development; the nurse will be more aware of differences and areas of caution that may need to be applied when working therapeutically with young people, and in particular when negotiating professional boundaries. Frankel (1998, p.128) emphasises when working with this age group it is important the therapist realises the vulnerability of the adolescent and the importance of the adolescent needing to have part of his life remain hidden to "protect or safe-guard the self". Safe guarding, or being able to protect

\footnotetext{
${ }^{2}$ Borderlme Personality disorder is a mental health diagnosis defined as "a pervasive pattern of instability of interpersonal relationships, self-image, and affects and marked impulsivity" (American Psychiatric Association: Diagnostic and statistical manual of mental disorders, 2000, p. 292).
} 
the self, is important for the adolescent experimenting with identity other developmental issues. It is essential nurses working with adolescents are aware of this and other developmental issues tor the young person.

Adolescents experiencing mental health problems need to be cared for by skilled clinicians aware of their developmental needs and very challenging behaviour. The therapeutic relationship and maintenance of therapeutic or professional boundaries with young people is contolex. The nurse having an awareness of the adolescent's developmental stage cannot be underestimated. Adolescents are separating from their parents, becoming closer to their peers with whom they experiment. They often display mixed emotions to a nurse with whom they are working, at times idealizing the nurse, while other times displaying rejecting challenging behaviour to her and their parents. This befhaviour has the potential to push boundaries within the nurse-adolescent relationship if the nurse is unaware of it. The therapeutic relationship and need for the nurse to maintain therapeutic boundaries is explored in more depth in the following chapter which discusses relevant literature around these issues. 


\section{CHAPTER 3}

\section{Literature Review}

\section{introduction}

This chapter reviews literature specifically relating to the therapeutic relationship and professional boundaries with adolescents in mental health nursing. I found very limited published literature and reseanch in this area. Throughout this chapter I have drawn heavily on the wark of Rene Geanellos, a noted adolescent mental health nurse researcher. Due to the limited published literature and research, I broadened the search to include therapeutic relationships and professional boundaries within adult mental health and paediatric nursing. Although there are some parallels and commonalities in these specialties, there are significant differences when comparing this literature to adolescent mental health nursing. Adolescent mental health nursing is unique and has its own set of challenges for the nurse and the adolescent. The nurse is attempting to understand the adolescent in terms of his developmental stage, but also understand his experience of mental illness.

Issues such as the use of the nurse's personality, friendship within the relationship, and the therapeutic use of self, all contribute the challenge of maintaining professional boundaries in the therapeutic relationship. The importance of the nurse using reflection about ther role within the relationship is discussed; this includes the learning about herself in context of these relationships and being clear whose needs; are met within the relationship. Opinions on what constitutes a therapeutic boundary are explored alongside the nurse's experiences of boutrdary violations. The effect the nurse's knowledge and experience has an iher clinical judgement is also discussed. 


\section{The literature search}

A literature search has been undertaken initially looking specifically at adolescent mental health nursing and the therapeutic relationship including professional boundaries. Very little literature was found in this area. The search was then broadened to explore adult mentall health nursing and paediatric nursing. The data bases CINAHL, PUBMED, and PsycINFO were utilised. Google Scholar was also used to explore this phenomenon. These databases were searched for literature relatied to adolescents, mental health nursing, the therapeutic relationship and professional boundaries. The following search words and phrases used were not limited to "adolescen* mental health nurs", "professional boundar", "Adolescen* and professional boundar", "mental thealth boundar", "therapeutic relationship". The word adolescentit was substituted by "youth", "young people" and "teenagers". The search focused on refereed journals initially; then extended to a New Zealand journal such as Kai Tiaki. In addition to this and perhaps more useful, I have utilised relevant references from articles and books sourced to inform this study.

\section{The therapeutic relationship}

The importance of the therapeutic relationship in mental health mursing has been well documented and researched (Cleary, 2003; Geanellos, 1998; Geanellos, 2004; Geanellos, 2005; Hinds, 1988; Horberg, Brunt \& Axelsson, 2004; Johansson \& Elkland, 2003; McCann \& Clark, 2003; Moyle, 2003; Peplau, 1988; Weissman \& Appleton, 1995). There are many nursing skills, qualities and interventions acknowledged and researched by these authors which enable therapeutic relationships to develop. These include; genuineness, trust, accessibility, companionship, support, stability, hope, nurturing, friendship, temmour, positive attitude, security, caring, reciprocity and concern. These skills and interventions are of importance in the nurse-adolescent relationship and in the development of therapeutic boundaries with adolescents. Therapeutic or professional boundaries are complex. They vary for each nurse and can differ with each patient (Carey, 1998; O'Brien, 2000). 
The therapeutic relationship is pivotal to adolescent mental health nursing. If requires the nurse to form an in-depth relationship with the adolescent which can be challenging. Geanellos (1997, p. 20) states "adolescents are often represented as antiauthority, antiestablistiment, undisciplined and difficult; they are rarely described as lovable". Because of this, forming a therapeutic relationship with this age group is not always easy and straight forward; it often requires the nurse to spend significant time with the adolescent engaging him in the relationship (Geanellos, 1997; Murray \& Wright, 2006; Ramjan, 2004). The nurse needs to have a genuine interest in the adolescent as this aids the development of the therapeutic relationship (Geanellos, 1997; Murray \& Wright, 2006). Adolescents are often very astute and can sense when a nurse displays a lack of sincerity and/or interest in his interactions (Murray \& Wright, 2006).

The forming and maintaining of the therapeutic relationship is the core of mental health nursing. It is the nurse's role to ensure the relationship is therapeutic. Professional boundaries play a significant role in ensuring this occurs. The management of professional boundaries is complicated but essential when engaging in relationships with adolescents.

\section{Defining boundaries}

An important part of the therapeutic relationship is the defining and management of therapeutic boundaries (Geanellos, 2003; PeterneljTaylor, 2002; Sheets, 1999). The boundary can be defined as the frame the therapist creates to allow the therapy to take place (Williams \& Swartz, 1998). The purpose of boundaries is to ensure practice standards are adhered to and both the client and therapist are protected (Williams \& Swartz). The significance of nurses managing therapeutic boundaries is of utmost importance. The adolescent's personality is in a stage of development which places him in a vulnerable position. This vulnerability is further increased when the adolescent is experiencing 
mental illness. The literature emphasises the managing the therapeutic boundary is the nurse's role rather than the patient's (Allenbach \& Steinmiller, 2004; Geanellos, 1997; Hawes, 2005; Scanlon, 2006; Totka, 1996).

Much of the research on therapeutic relationships discusses the value of nurses adopting an informal, friendly approach to the relationship, with patients in mental health (O'Brien, 1999; Geanellos, 1997; Scanlon, 2006). However, finding the balance between this and a relationsifip that remains professional, with clear boundaries is often a challenge. The mental health nurse frequently faces the challenge of finding a balance between ensuring she portrays a professional front to the patient yet sharing enough of herself to connect as a person (Gastmans, 1998, O'Brien, 1999).

One of the challenges for mental health nurses is determining how much she should share of herself with the patient, as sharing of self is: what happens between friends. The concept of friendship and friendfiness has been widely discussed in nursing literature (Ford \& Turner $_{\text {, 2001; }}$ Geanellos, 1997; Geanellos, 2005; Hawes, 2005; Kimberly, 2002). Friendship can be understood as a mutually reciprocal relationship where both needs are met. Although the nurse has a lot to gain from the relationship she has with the adolescent (Geanellos, 1997), it is tought, the relationship in mental health nursing should be meeting more of the patient's needs rather those of the nurse. This relationship exists win a professional context (Geanellos, 2005). Hawes $(2005$, p. 16) found a blurring of nurse-patient boundaries occurs "when the relationship slips into a social context. where the nurse's behaviour reflects his need at the expense of the patient's needs". The excessive discussion of a murse's personal life, her problems, worries, gossiping and social talking have all been identified as a crossing over of professional boundarites; as discussions of this nature can indicate the nurse's needs forefront rather than needs of the patient (Allenbach \& Steinmiller, 2004; Gastmans, 1998, Sheets, 1999). 
The nurse is a person too, with feelings, emotions and experiences similar at times to the patients she works with (Scanlon, 2006) which cannot be ignored. Yet, it is the nurse's role to utilise her skills ensuring she maintains a professional, yet therapeutic relationship with the adolescent. This is supported by the collaborative work currenty being carried out by the Australian Nursing and Midwifery Council, Nursing Council of New Zealand and Faculty of Health for the University of New Castle (2007) in validating a set of guidelines on boundaries: of professional practice for nurses and midwives.

The complexity of professional boundaries is compounded when what constitutes a professional boundary, for one nurse, may be too striet: for amother (Totka, 1996). A nurse may also disclose more about herself to ome adolescent than she would to another, depending on the needs of the adolescent, and the relationship she has with him. The variation of the boundary and constant movement adds to the complexity. PeterneljTaylor $(2002$, p.28) suggests "when it comes to boundaries, there are no black and white answers to the tough questions posed by the dilemmas in practice. Instead, shades of grey cloud my thinking. Mamaging boundaries is often easier said than done". When engaging with; the adolescent in a therapeutic relationship the nurse needs to keep: the need for boundaries in the forefront of her mind.

For the nurse to effectively engage in relationships with patient she needs to become involved with them (O'Brien, 1999). To achieve; this she also needs to be available to the relationship, showing she is open and accessible to receive communications and have interactions withithe crient. To engage with an adolescent the nurse needs to make connections with him, this requires a genuine interest in the adolescent (Murray \& Wright, 2006). When nurses are able to communicate a genuineness and sincerity to the adolescent it provides an avenue for the rapport and the therapeutic relationship to develop. Rogers (1980, p.153) found when this occurs "the message comes through the the 
recipient that "this other individual trusts me, thinks I'm worthwhile. Perhaps I am worth something. Perhaps I could value myself. Perhaps I could care for myself". This sincerity from the nurse can suppont the adolescent in feeling worthwhile. It often requires the therapist or nurse to give something of herself to the relationship which helps in rapport building enabling the adolescent to feel connected to the nurse. This assists in ensuring the relationship is meaningful which helps the adolescent to express himself and tell his story (Murray \& Wright, 2006).

\section{The Nurse's Personality}

The concept of the use of self as a therapeutic tool is discussed extensively in mental health nursing (Carper, 1978). Geanellos (1997, p. 22) states "the self refers to all the nurse brings to her interactions; with others; it encompasses the dynamic entity she has become the knowledge, skill and wisdom garnered through her experiences of fiving and how she operates in the world." The nurse is unable to genuinely separate herself and her personality from the work she does with patients. When the nurse does attempt this she betrays her persanality and presents a front to the patient that is artificial and lacks genumeness (Scanlon, 2006). The importance of the nurse showing sincerity and genuineness to the adolescent is crucial in the development of the therapeutic relationship. However, this concept of the nurse using her personality in the therapeutic relationship can bring potential problems. These problems include too much self disclosure when the nurse is attempting to engage with the adolescent. The need for the nurse to know herself and have the capacity to continue to work through her own personal issues when working with patients in mental health is: well documented in literature (Geanellos, 1997; Scanlon, 2006). Nurses in this area are working with vulnerable patients because of their mental health issues. If a nurse is not aware of her own issues related to her mental health these may become confused with the patient's and be potentially detrimental. 
A nurse who reflects on her practice and recognises issues that relate to herself rather than the patient, is more effective in the relationship. Scanlon $(2006$, p. 325) states "skills involved in forming therapeutic relationships require interpersonal skills that can be learned, but the learning tends to be in the form of learning about oneself". When the nurse can appropriately share experiences about herself, using clear professional boundaries, much can be gained (Geanellos, 1997). However the emphasis should always be on whose needs are being met with this sharing - the nurse's or the patient's? Gastmans (1998) motes the importance of nurses ensuring the nurse patient relationship diaesn't become an arena in which the nurse discusses her own problems, concerns and expectations. Therefore constant vigilance is requized by the nurse. Adolescents experiencing mental health problems have enough issues to contend with without taking on the nurse's problems and helping to solve them. Yet, when the nurse is able to demonstrate self awareness and a capacity to look at her own issues her use ofi self can benefit the adolescent. For example, if the nurse is able: to appropriately share how she managed a stressful situation as the adolescent is searching for role models and learning experiences; with which he can learn from. The therapeutic relationship with a nurse: who is open to sharing appropriately about herself can assist in this process and in joining with the adolescent in the relationship. Geanellos \$1997, p.27) states

I certainly told a lot of stories about growing up in my family and the trials of my own adolescence. I think this type of reciprocity is important, although it needis: to be appropriate to the context and the adolescent, because it helps reduce the distance between you and the adolescent.

She also emphasises the importance of the nurse being clear about who the adult in the conversation is. Because the nurse may experience stressful events in her own life, there may be times when the nurse is not vigilant and makes mistakes by sharing too much information with the adolescent. 
Peternelj-Taylor (2002, p.24) found "as human beings we are all vulnerable, and because of our humanness, we struggle with the meaning of boundaries in therapeutic relationships". When a nurse is attempting to engage a patient and present a more human, accessible, and informal sense of self in the relationship there is a danger of this crossing the line (O'Brien, 1999). The nurse needs to be aware of her role in the therapeutic relationship, while having an understanding of the inevitable power differential that exists between the patient and the murse (Peternelj-Taylor, 2002). The power differential emphasises the potential increased vulnerability of the patient and the need for the nurse to remain professional in his role within the helping profession. Gastimans (1998, p.6) states "Nurses should not speak to the patient in the same way they speak to family or friends, even if that would be much more easy or enjoyable". Because nurses are people with their awnil life experiences, they will make mistakes such as falling into social talking and friendships with adolescents. This often occurs when the nurse is sharing something of herself in interactions. Nurses can learn through these mistakes and this learning could be shared with others.

\section{Nurses Learning}

Totka (1996) found that if nurses were able to talk about professional boundaries, and the challenges in managing these openly among their peers, this may foster more open discussion around these chalfenges which may assist in nurses' learning. It is also thought that more experienced nurses who are role models to their peers, can often make more effective decisions around the therapeutic use of boundaries within the nurse-patient relationship (Allenbach \& Steinmiller, 2004; Hawes, 2005; McCutcheon \& Pincombe, 2001; Peternelj-Taylor, 2002; Totka 1996). If these nurses were able to share their stories with junior murses of these experiences and encourage open communication around boundaries, valuable learning opportunities for all nurses would be more available. There are no clear specific guidelines in relation to professional boundaries that apply in every nursing situation. Support 
from: nursing peers in managing the complex issue of professional Goundaries is helpful.

Another opportunity to learn about professional boundaries is when murses make mistakes in managing the boundary, and therefore leam from the experience (Hawes, 2005; Totka, 1996). Peternel-Faylor (2002 p.25) found "arriving at decisions involving therapeutic boundaries requires knowledge, experience, and clinical judgement. Boundary work is not simply an exercise in logic, but rather one rooted in therapeutic integrity". Strong nursing leadership which creates a culture of openmess and sharing among nursing staff, plays an important role in ensuring nurses are supported in the decision making process in regards: to therapeutic boundaries within the nurse-patient relationship (Allenbach \& Steinmiller, 2004).

Addelescent mental health nursing is a complex and specialised area of . nursing. As a specialty it remains an area of nursing which is largely \&nresearched and unpublished (Geanellos, 1999; Hodgkinson, Watson, Wadge, Abeles \& Danquah, 2005). There are further research gaps when searching for any New Zealand research related to this field of practice.

\section{Conclusion}

As: the literature states it is the nurse's role to maintain therapeutic boundaries in mental health nursing. When the nurse engages with the adolescent in a therapeutic relationship she needs to be acutely aware of the role boundaries play within this relationship. The nurse uses herself as: a therapeutic tool. She shares her personality with the young person, and discloses appropriate information about herself in the relationship. Many researchers found friendliness and friendship has significance in this relationship; however this is not a reciprocal friendship where the nurse talks about her worries and problems. The nurse has the adolescent's needs at the forefront of her mind when working 
therapeutically with the adolescent. A culture of sharing and fionesty amongst a nursing group could support nurses in learning from mistakes that may occur when the nurse crosses the boundaries with: an adolescent. Strong, supportive nursing leadership needs to allow this to occur. As this literature review illustrates there is literature: on therapeutic relationships and professional boundaries in other areas of nursing, however, the literature related to specifically to adolescent mental health nursing is limited. It is hoped that this research thesis; exploring senior nurses' experience of assessing, understanding: and working with therapeutic boundaries when working alongside adolescents in a mental health setting will contribute to mursing knowledge in this complex area of practice. 


\section{CHAPTER 4}

\section{Methodology Chapter}

\section{Introduction}

This research project has used a qualitative method, narrative inquiry, with an interpretive approach and a specific framework for analysis. The researcher has many obligations when undertaking research. The research process needs to be easily understood, the methad and methodology need to be congruent with the theoretical underpinzings of the project ensuring the question can be answered adequately. The research process needs to be ethical and appropriate. When contributing to nursing knowledge through research, the processes of the research need to be rigorous and congruent to the topic researched. The research methodology and design needs to be compatible witth the research question, as this helps to determine whether or not the findings are valid and reliable. The considerations also include practicalities such as project size, participant recruitment, and costs. It is important that the context of the project and research methodology is understood. Throughout the discussion I explain how I undertook this project of exploring nurses' experiences of therapeutic boundaries with adolescents in mental health using narrative inquiry.

The chapter begins with a brief discussion of nursing research, including the main paradigms used. The philosophical underpinnings of the Constructivist paradigm are discussed and how narrative inquiry ${ }_{n}$ as: an interpretative research approach, fits into this domain. The way rarrative inquiry has been used as a specific research design to explore rumsses' experiences is explicated. This includes data collection, management and analysis processes. 


\section{Nursing research}

Nursing research has existed since the 1800s when Florence Nighthingale published Notes on Nursing in 1859 (Polit \& Beck, 2006). Nurses are engaging in research increasingly, as the profession develops there is a focus on nurses being able to provide evidenced based practice to patients/clients (Polit \& Beck, 2006; Schneider, Elliott. Beanland, LoBiondo-Wood \& Haber, 2003). Evidence based practice contributes to patients/clients receiving the best possible nursing care available to them. Research helps to validate the effectiveness of nursing caxe in a world where there are considerable pressures on healthcare funding. Nurses are accountable for ensuring their practice is cost-eftective, clinically appropriate and has the best outcomes for patients/clients (Polit \& Beck, 2006). Nursing research requires methodological considerations that are appropriate to its own practice context.

The Positivist paradigm and the Naturalistic (or Constructivist) paradigm are the two world views mainly used in nursing research, although these are not the only paradigms from which nursing research originates; (Polit \& Beck, 2006). The Positivist paradigm is defined by Polit and Beck (2006, p.507) as "the traditional paradigm underlying the scientific approach, which assumes that there is a fixed, orderly reality that can be objectively studied". This approach is usually associated with quantitative research. Quantitative research designs include but are not limited to studies ranging from descriptive designs to comparing groups and experimental designs (Roberts \& Taylor, 1998).

The naturalistic or constructivist world view is defined by Polit and: Beck (2006, p.504) as often having multiple realities or interpretations of realities, "the goal of research is to understand how individuals construct reality within their context; often associated with qualitative research". Geanellos (2004) believes mental health nursing is based on the formation of relationships and the ability of nurses to understand tuman 
encounters and experience, these understandings often requuire: a qualifative research approach. She points out the importance of nursing developing knowledge of its own, rather than borrowing from other disciplines. She recommends using qualitative methods will further develop nursing knowledge and therefore advance the practice of mental kealth nursing. However, there is no 'best research design' when undertaking nursing research. The research design and methodalogy meeds to be based on the most appropriate design to help answer the question posed (Roberts \& Taylor, 1998).

In this project narrative inquiry, a qualitative research approach has been used to explore nurses' experiences of assessing, understanding and working with therapeutic boundaries when working alongside adolescents in a mental health setting. This interpretive methoeflogy respects the nurses' experiences of exploring their understanding of the significance of working with therapeutic boundaries in mental health

\section{Qualitative methodology}

This section illustrates the use of a qualitative research methodiology, narrative inquiry, in exploring the nurses' experience of therapeutic boundaries. A brief explanation of the theoretical perspective which underpins this methodology is given. The relevance of a constructivist approach in exploring this phenomenon is discussed, particularly interpretative design and how narrative inquiry sits within this apprøach. The significance of culture and experience are also explored within this methodological approach. The use of an interpretative design for mursing research is explained and specifically for this study. The discussion begins with the interpretative approach.

\section{An interpretive approach}

When exploring and attempting to understand how nurses work with therapeutic boundaries when alongside adolescents in mental health, an in-depth exploration of the nurse's experience is required. The 
philosophical underpinnings of the constructivist paradigm respect this type of exploration.

The constructivist paradigm view of 'meaning' is that it is constructed by people when they engage with the world they are interpreting. The meaning is neither objective nor subjective but both of these realities are held together (Crotty, 1998). Crotty (1998, p.42) described constructionism as "the view that all knowledge, and therefore all meaningful reality as such, is contingent upon human practices, being constructed in and out of interaction between human beings and their world, and developed and transmitted within an essentially social context". Constructivism also supports the notion that each person's experience is unique and individual's interpretation of the world is just as "valid and worthy of respect as any other" individual's interpretation, therefore there is no one correct reality, multiple realities exist (p.58)

Therefore, when interpreting a nurse participant's experience, it is just that, an interpretation of what $\mathrm{I}$, as the researcher, think may be occurring. My interpretation may differ from that of the reader's interpretation. However, both interpretations are considered useful in this project interpretive research aims to make sense out of the experiences described by the participants.

Narrative inquiry is an interpretative research methodology which sits in the constructivist paradigm, as it is impossible to separate the stories from the lives of their narrators and the communities within which the stories are told. Narrative allows stories to be constructed and shared as people engage with the world and their experiences in it. It then allows participants and researchers to interpret these experiences.

\section{Narrative inquiry}

As a research methodology, narrative inquiry fosters reflection and preserves knowledge, while respecting the participants' thoughts, culture 
and history. Narrative seeks to encapsulate the significance of the work nurses are already doing, as the emphasis is on the nurse's experience and everyday practice (Benner, 1991; Bruner, 1984; Carson \& Fairbairn, 2002; Clandinin \& Connelly, 1991; Denzin, 1989; Koch, 1998; Polkinghorne, 1995; Sandelowski, 1991).

Narrative inquiry was first discussed in the 1930s. Initially coming from a literary perspective, it has mainly been used in the social sciences (Wood \& Giddings, 2003), for example philosophy, sociology and psychology. Emden (1998, p.30) found, although narrative inquirry is relatively new in the field of nursing it is becoming increasingly utilised as a research approach. She states "it does not have any single heritage or methodology and its practitioners draw upon diverse sources: of influence". Woods and Giddings (2003) found the narrative approach has been strongly influenced by history, phenomenology and hermeneutics. Phenomenology is the study of the lived experience, or of things within human experience (Roberts \& Taylor, 1998). Hermeneutics is a process of interpretation often used in research to understand human practices and events (Crotty, 1998). As the focus of narrative inquiry is to explore, understand and interpret the experience of the participants, the link to both of these methodological approaches is apparent.

The term narrative is often used interchangeably with 'story' in qualitative research (Emden, 1998; Gudmundsdottir, 1995). Gudmundsdottir (1995) suggests that a flexible use of the terms narrative, and story, is often more useful to researchers, as it enables a wide range of social phenomena to be analysed. Emden (1998, p.35) explains "although narrative clearly involves stories, it is more than a single story ... the term embraces the collective 'storied wisdom' of people's individual stories". In this study I refer to the data collected from the interviews as the nurse's narrative, this includes the whole experience. At times the term story is used in relation to individual stories within the nurse's narrative. 
For this project I have drawn on the work of Clandinin and Connelly (2000) who utilise narrative inquiry from an educational perspective. Their focus is on experience; from which they conduct a narrative inquiry. Clandinin and Connelly (1990, p.2) define narrative as both" "the phenomena and the method". Narrative inquiry endeavours to make an in-depth exploration of human experience and then understand this experience. As an interpretive methodology, narrative attempts to explore, understand and explain both human and social realities (Crotty, 1998). My research explores the experience of the nurse in assessing, understanding and managing therapeutic boundaries when working with adolescents in mental health. The context to the research topic is: also highlighted.

The work that nurses do in forming relationships and defining boundaries with adolescents is significant in terms of the skills and knowlledge required, the use of narrative inquiry highlights this significance. Gudmundsdottir (1995, p.31) states:

By using narrative form we assign meaning to events and invest them with cohierence, integrity, fullness, and closure. When we place events drawn from our experiences within an order provided by narrative, we also invest them with a moral significance.

Story telling is used by individuals to construct and express meaning (Mishler, 1986). Practice stories help the nurse to reflect on herr work with the adolescent; the therapeutic relationship, her role within the relationship, and the goals the nurse and adolescent are aiming to achieve. Stories allow us to explore the world and our place and! role within it, "stories call us to consider what we know, what we hope: for, who we are, and what or whom we care about" (Witherell, 1995, p.40).

The participant brings with her the story of her past, present and future (Bruner,1984; Clandinin \& Connelly, 2000; Denzin, 1989) which is captured by the telling of her experience; in this case working: with adolescents in the mental health setting. Crotty $(1998, p .64)$ suggests "when we narrate something, even in telling our very own story, it is (again in the normal course of events) the voice of our culture - its many 
voices, in fact - that is heard in what we say". Each participant in this research will have differences in her culture and a different experience of her work with adolescents; the use of narrative inquiry respecks this difference. Every nurse's experience is unique, her past expenience, beliefs and culture have an effect on the work she does with adolescents in a mental health setting.

\section{Experience and culture}

The way a person behaves in the world, his customs, beliefs and values are what define his culture. Every experience a person has influences that person's future experiences and actions (Dewey, 1938). Dewey/( $p$. 27) found "from this point of view, the principle of continuity of experience means that every experience takes up something from those which have gone before and modifies in some way the quality of those which come after". Each experience a nurse has in the relationship witio an adolescent will influence or impact on her future experiences, bothi with that adolescent, and others. Narrative inquiry provides a way to explore this experience.

The effect a person's culture has on her experiences and interpretation of these experiences is important in narrative inquiry. Culture helps to shape the way in which we think about the world and the way in which we function and behave (Crotty, 1998). The use of a narrative methodology in research provides opportunities for the participant to explore cultural influences, and the effect these may have her experiences personally and professionally (Bruner, 1984; Bruner, 1991; Clandinin \& Connelly, 2000; Crotty, 1998; Dewey, 1938).

Narrative inquiry as an interpretive methodology provides a research approach which is respectful to the participant's experience, culture and way in which she sees the world. It allows the person's story to be told incorporating life experiences, values and beliefs. In this research it provides an opportunity for nurses' stories to be heard. 


\section{Narrative in Nursing}

This section highlights the value of using a methodology such as narrative inquiry for nursing research and how it enables murses' experiences to be heard. It explores the place of story telling in mursing and within nursing research. Narrative inquiry provides an opportukity to look at what nurses are already doing as the emphasis is on atursing practice. Carson and Fairbairn (2002, p.3) state, "research using narratives is more respectful of nursing practice since it does not seek to impose a pre-existing methodological framework upon it, and takes seriously the stories that practitioners tell about what they do".

The use of narrative or storytelling can make nursing practice visible (Koch, 1998). The very telling of stories provides a space for the murse to reflect on her practice, to acknowledge where she may have gone wrong but also what was done effectively, this facilitates development of her practice (Benner, 1991). Narrative inquiry enables an in-depth exploration of the nurse's experience and practice. Benner (199k, p.4) found narratives "capture the significance of the practice and narkatively demonstrate meaning-laden clinical episodes that convey the worth of the work". Through the use of narrative inquiry the nurse's personal experience is able to be captured and explored (Lieblich \& Josselson, 1997).

Working with adolescents is complex. Using a narrative methodalogical approach enables a deeper understanding of the phenomena of not only the nurse's practice, but how the nurse's personal world view, culture and identity influence her practice. Using narrative inquiry provides the nurse participants in this research, an opportunity to reflect on their everyday practice of forming relationships, managing therapeutic boundaries and the complexities that this entails. When nurses have the opportunity to tell practice stories, meanings and feelings related to 
practice knowledge are revealed, which can have an influence on future nursing knowledge and practice (Benner, 1991).

The utilisation of narrative inquiry enables the practice of nurses; to: be visible and heard. Telling practice stories is an opportunity to reflect on practice, which aids the development of practice. Narrative provides nurse researchers with an opportunity to explore the participants' experience in an in-depth way, while ensuring the participants' voices; be they patients or nurses are heard. The knowledge the nurse participants possess in this study can be shared through a narrative approacth and contribute to the existing body of knowledge. This knowledge may benefit nurses from a variety of practice settings, who are working; with adolescents experiencing mental health problems.

Having established that narrative inquiry is the best approach to use in this study I now move on to the research plan for design, scope amdi data processes used. There are various ways to collect data using nanrative inquiry and various ways to analyse and interpret it. The following section discusses the processes used for this study to understand nurses' experiences of assessing, understanding and maintaining therapeutic boundaries in a mental health setting.

\section{The Research Plan}

The next part of the methodology chapter looks at how I went aboxt this research project using narrative inquiry and the considerationsis and processes entailed. Firstly the design and scope are discussed; secondly, ethical considerations; thirdly, data processes; and tinally, strategies for rigour. In brief, I met with four participants, using a semistructured interview to collect the data. Clandinin and Connelly's (2000) three dimensional narrative inquiry space is used for data analysis. Lincoln and Guba's (1985) four criteria for determining rigour: in qualitative research is also used. 


\section{The project}

The aim of the project was to understand nurses' experience of assessing, understanding and working with therapeutic boundaries when alongside adolescents in mental health. I had to define whe the participants would be, the method of recruitment, the scope of the project and the ethical considerations.

\section{Scope and size}

This project is the research component of a master's degree and geeds to be completed in one year. For this reason and the chosen methodology I felt a maximum of four participants would enserse the project was manageable.

\section{Participants}

I had to identify who the participants would be, the inclusion or exclusion criteria, recruitment method and plan of contact and communication. The participants for this study must be Registered Nurses in New Zealand who are currently employed in a District Health Board in Child and Adolescent Mental Health, working specifically with adolescents.

\section{Inclusion Criteria}

The inclusion criteria stated the nurses had to have at least three years experience of working in an adolescent mental health setting. The aim of this study is to explore how nurses understand and work with therapeutic boundaries when working alongside adolescents by asking the nurses to talk about their experience. Benner and Wrubel (1989) emphasise the need for expert nurses to help inform nursing theory, three years in a chosen specialty will help to ensure the nurse has relevant knowlledge and experience. It is also thought that experienced nurses are able to discuss their practice in much more depth which will bring about further understanding of this phenomenon (Benner, 1991). 


\section{Recruitment}

There are a small number of nurses in New Zealand working in childiand adolescent mental health, consequently we know one another. Snowball sampling, as the method of recruitment, was used in this project. Snowball sampling is frequently used when there is a small group of possible participants or when participants who meet the inclusion criteria are difficult to identify (Polit \& Beck, 2006; Schneider et al, 2093). A small amount of potential participants are identified, these petential participants identify other possible participants, and this grows like a snowball.

\section{Process}

I began the recruitment process by emailing a covering note key professional networks in New Zealand attaching the information stheet for participants (Appendix 1) along with the consent form (Appendix: 2) requesting they pass this on to any nurses that met the inclusion criteria. The potential participants then emailed me their interest in participating in the study. I replied via email requesting the nurse send me the signed consent form by mail. I offered to telephone the nurse to answer any questions she may have. The participants were chosen on a first come first served basis, although consideration was given to the geographical area of the participant, as the use of face to face interviews meant I needed to consider the time and expense involved. On receiving; the completed consent form I arranged an interview date and time with the participant at a place and time convenient to her.

\section{Ethical considerations}

As a researcher studying human experience it is essential that I comsider the ethical implications for the participants in order to minimize harm to the participants and ensure informed consent is obtained. Ethical considerations include ensuring the participants and their stories are respected by providing confidentiality and/or anonymity. The researcher also has to consider issues such as conflict of interest, coercion, 
exploitation, benefit and risk. In New Zealand the researcher's obligations under the Treaty of Waitangi are part of the project.

\section{Ethics approval}

When research is conducted in New Zealand with human participants the researcher has an obligation to obtain approval for the proposed research from a suitably recognised ethics committee. The ethics application process is extensive and rigorous. I sought and received ethics approval from the Victoria University Human Ethics Commitiee for this research project. Appendix 3 contains the approval letter from the committee.

\section{Treaty of Waitangi}

When living and working as a health professional in New Zealand, acknowledgement of the Treaty of Waitangi is mandatory. This is the founding document of New Zealand, which aims to ensure that Maori (the indigenous people) and European are equal partners within the country. Therefore involvement of Maori in all things related to the health of Maori is significant (Durie 2001).

The Health Research Council of New Zealand (1998) states for best practice consultation with Maori should start at the beginning of the research process; this will initiate participation by Maori in the entire process, which will ensure effective Maori input. As I identify as NonMaori, it was important for me to ensure consultation with Maori at the beginning of the project. I also needed to consider the identity of my participants who may belong to other cultures.

During the design phase of my project I undertook consultation with the Te Whare Punanga Korero Board, the Maori advisory team for Taranaki DHB where l'm employed. The purpose of this consultation. was specifically requesting they consider whether my proposed research would be culturally safe and appropriate for Maori. If any participants 
identified as Maori I needed to have thought about the specific reeds and processes that should be followed specific to Maori. This ensures I meet my obligations under the Treaty of Waitangi and cultural safety in the Nursing Council of New Zealand competencies 1.2 and 1.56 (Nursing Council of New Zealand, 2005). The Te Whare Punanga Korero Board gave full support to my research project with a support letter which was included in my ethics application.

\section{Confidentiality and Anonymity}

It is the researcher's responsibility to ensure the confidentiality and/or anonymity of the participants is respected (Robert \& Taylor, 1998). The data needs to be stored safely with access to authorized people only. $A$ pseudonym should be used for any information that requires identification such as the transcribed data (Roberts \& Taylor). If the raw data identifies the participants in any way the researcher needs; to protect their anonymity. This may include making alterations to the data or changing details to ensure the participants can't be identified. It is essential that the researcher makes every attempt to respect the individual participant's rights to confidentiality and anonymity.

The participants' confidentiality has been maintained to the best of my ability throughout the project. I am the only person aware of whom the respondents were. When I submitted my research proposal to the Ethics Committee I received feedback about whether I was able to guarantee confidentiality to the participants. This was due to my stating "there are a small number of adolescent mental health nurses in New Zealand, consequently we know one another". The Committee pointed out that I may not be able to guarantee confidentiality to the participants as the participants' opinions and narrative may be recognisable by their peers. This would be common in a small country with a small population such as New Zealand. The committee felt the participants had a right to be aware of this prior to agreeing to participate. I altered my information sheet accordingly to read: 
due to the small number of adolescent mental health nurses in New Zealand, and the narrative design of the research, there is a possibility that participants' views/experiences may be recognised by their peers.

No identifying features have been used and the names and geographical location of the participants is confidential.

For the safe and confidential management and storage of the data the audiotapes of the interviews were labeled with a pseudonym. The audiotapes were transcribed by a transcriber employed for that purpose she understood confidentiality issues and signed a confidentiality statement. The transcriber and I, as the researcher, were the only people to have access to the raw data to ensure the participants' confidentiality was protected (Roberts \& Taylor, 1998). All written material is kept in a locked file with restricted access and all electronic information is in a password-protected file.

\section{Informed Consent}

Informed consent was obtained from the participants after an interest was shown in participating in the research via email. The participant information sheet was emailed to each participant with the consentifom. The information sheet provided a comprehensive explanation of the study and their potential participation. The participants were givern an opportunity to discuss any questions they had regarding the research and their participation. Signed consent forms were returned to me: via the mail prior to the participants' interviews. An opportunity wass also given to the participants to ask any further questions when the interviews were arranged.

\section{Risk and harm}

It is the researcher's responsibility to ensure potential ham to participants is kept to a minimum. Harm or distress to participants could potentially occur in this study if a nurse had experienced a distressing relationship with an adolescent in her care. Discussion of this 
relationship may re-traumatise the nurse. A nurse may also disclase: an unresolved or inappropriate relationship or boundary crossing with an adolescent. If this had occurred during the interview I planned to stop the recording of the interview immediately and support the nurse: as appropriate. If the issues were unresolved at that point, the nurse was given the option to withdraw from the research. Whether or not this option was taken, the nurse would have been encouraged to take the issues to clinical supervision or be referred to the Employee Assistance Programme in her organization.

\section{Summary}

There are clear guidelines that need to be followed when undertaking research. It is essential the research process is congruent within the methodological underpinnings of the study. The research must be ethical, and appropriate procedures related to this, must be follawed. The participants need to be aware of the research process and their rights. It is important that their confidentiality, safety and cultural needs are met.

\section{Research Processes}

The third part of the methodology chapter focuses on the processes of data collection, management, analysis, and rigour used in this project. The data collection method used is the unstructured interview. Next the management of the data is outlined. The characteristics of the participants, process reflections, and the role of the researcher in narrative inquiry is also discussed. The data analysis process used is Clandinin and Connelly's (2000) three dimensional narrative inquiry space. Strategies used to ensure the research process is congruent and rigorous are explained. Lincoln and Guba (1985) identify four criteria: for determining rigour in qualitative research; these criteria are explained along with their relevance in this study. I discuss the researcti: and method processes generically as well as specifically to this project. 


\section{Data Collection}

This section explores data collection methods used in narrative inquiry. It focuses on the unstructured interview as the method utilised to collect data in this study and the rationale for its use. The role and influence of the researcher must also be considered. When utilising narrative inquiry as a research methodology the data collection methods can include indepth conversations or interviews. These can be on either one occasion or a series of conversations over time. In this approach the emphasis is on obtaining the participants' accounts in their own words or terms.

\section{The unstructured interview}

The unstructured interview is viewed as the most effective data collection method when using a narrative design, due to its ability to allow participants more open responses (Lofland, 1971; McCance, McKenna \& Boore, 2001; Polkinghorne, 1988). An unstructured interview allows the participant to tell her story speaking in her own voice, and having control of the topics discussed and the direction the narrative takes Mishler, 1986). The in-depth interview is complex, it provides a leaming experience which is rich and would be difficult to communicate thy in another context (Paget, 1983). The unstructured interview allows fior indepth exploration into the nurse's experience of working with boundaries with adolescents.

An awareness of ensuring I give space and time to the participant to tell her story, noticing how I, as the interviewer, contribute to the narrative, what I say, when I speak and the dynamics that my role creates in the process are valuable.

When conducting the research the position of the researcher is not able to be an objective one. The use of a reflective journal helped me to reflect on, and process my ideas, it also ensures my bias and opinions are acknowledged. As the researcher, I become part of the process having lived my own story as an adolescent mental health nurse, and my 
personal life story. Prior to the interview process I was aware of the importance of responding as the researcher and not the participant's nurse.

Lofland (1971, p.76) describes the unstructured interview as an opportunity for the interviewer to elicit from the participant, what she considers is important information or questions on the given topic, rather than expanding on the information the interviewer feels she afready knows. "Its object is to carry on a guided conversation and to elicit rich, detailed materials". The interviewer need not be highly skilled or knowledgeable about the topic being discussed and explored, as is the participant's opinions and knowledge that is sought through the use of the unstructured interview (Mishler, 1986).

\section{Reflexivity}

My role as the interviewer and investigator is important in the research. As the investigator, interviewer and analyst of this research I have: an agenda that is my focus to investigate. Sandelowski (1986, pi30) proposes "the credibility of qualitative research is enhanced when investigators describe and interpret their own behaviour and experiences as researchers in relation to the behaviour and experience of subjects". Geanellos (1997) discusses the need for the researcher to acknowledge her own beliefs and pre existing understandings of the phenomena; she suggests doing this using a reflective journal. Overcash (2004) notes the importance of the researcher not responding to the participants: as a nurse attempting to help solve the participant's problems, but being consciously aware of remaining in the position of the researcher.

It is helpful for researchers to keep a reflective journal throughout the research process (Connelly \& Clandinin, 1989) as this contributes to the rigour of the research. The researcher's opinions are brought to consciousness and attempted to separate those from the participantis. 
The need for clarification of the role of the researcher prior the interview process is significant in the use of the unstructured interview when undertaking narrative inquiry. The importance of the interwiewer's awareness of her contribution to the interview is also significant Paget, 1983).

\section{The interview process}

My experience of working in unit which set up as bicultural (Maoni and non-Maori) emphasises the importance of mihimihi/introductions: and having an understanding about where the people I am working with originate, in terms of their culture and identity. The engagement process with adolescents and their families is significant. I wanted to take this awareness into the interviews.

For Maori in New Zealand the significance of whanau/family and where the individual comes from is important. Individuals are seen first as members of a whanau or Iwi. It is just as important for the patienti and family to have some idea about who I am and where I have com. This enables the family to have a sense of my history which aids in determining who I am as a person and whether or not I am safe share personal information with. I see this as equally important wher asking participants to contribute my research. The interviews were therefore initiated using mihimihi, which included an introduction to who $1 \mathrm{~m} ; \mathrm{my}$ identity and brief overview of my nursing experience. I invitud the participant to share with me a brief narrative of who she is, where she originated and how she came to work in this field. This aided $\mathrm{me}_{\mathrm{n}}$ as the interviewer, in positioning the participant in regards to her cultural context, identity and past experience at the start of the interview. Bruner (1984, p.5) supports this when studying anthropology, she states "stories cannot be viewed simply as abstract plot structures isolated frome their cultural context. We know that stories must be seen as rooted in society and as experienced and performed by individuals in cultural settiruss." 
W was important the participants in this study felt valued and their stories respected. The stories in this research belong to the participants asitheir information and experience. As the researcher, I had the privilege of sharing part of this experience with them. Clandinin and Connelly (2000, p.110) found "the way the interviewer acts, questions and responds in an interview shapes the relationship and therefore the ways participants respond and give accounts of their experience". Engaging in some conversation with the participant in response to her stories thraughiout the narrative allowed a greater depth of explanation of the sturse's experience to occur.

Following the mihimihi/introductions process with the participants if used an opening statement and prompts as necessary. The opening statement provided a beginning point for the participant; it also gnuminded the interview in focusing on the reason for the interviews. The prompts were used as a guide and a tool to help keep the interview focusedion the phenomena being discussed.

The interviews provided rich and enlightening data on murses' experiences of assessing, understanding and working with therapeutic boundaries with adolescents in mental health. The data from the interviews needed to be managed effectively.

\section{Data management}

The interviews are audio-taped to ensure an accurate representation of the interview and to ensure the researcher has the opportunity to capture the experience. The interviews were audio taped with two separate recording devices to minimise any taping error. The audiotapes were labeled with a pseudonym to protect the participants' identity and stored in a locked cabinet to prevent access by unauthorised people (Roherts \& Taylor, 1998). The access to the data was limited to those involved in carrying out the research and those employed for specific duties related to the data management, for example, a transcriber. 


\section{Characteristics of the Participants}

Four participants, three female and one male were interviewed for this study. Of these, one nurse was Pacific Island, one English, one Australian born New Zealander and one New Zealand European. The age range was one in her 20's, one in 30's, one in 40's and one in 50's. They were employed in three District Health Boards (DHB). Two nurses are in inpatient settings, and two in community nurse positions: one community nurse previously worked in an inpatient setting. All the participants had at least four years experience in adolescent mental health; the most experienced had 15 years. Three participants' undertook their nursing education in New Zealand with no overseas experience and one in England. None of the participants had managerial responsibility.

\section{Process reflections}

The way in which I conducted the interview process and my role as the researcher are discussed in this section. Following each interview I made a journal entry to record my thoughts and reflections of the interview. This helped me to think about my role as the researcher. The way $I$ asked questions in the interview could potentially have an effect on the practice stories told. This section summarises my reflections on the processes 1 used in the interviews and highlights issues.

The interviews were arranged as convenient to the participants. All the participants initially chose to have the interviews in their workplace in work time. I wondered if this reflected the topic of professional boundaries and the desire to leave work discussion at work, in work time, and not brought into the nurse's personal life. One interview did occur at the nurse's home due to a mix up in the interview date and it was convenient to her at the time. Another interview also had to be rearranged at short notice as when I arrived the participant was enroute to a crisis visit. 
Each interview began with the mihimihi process. This varied slightfy with each participant. I noticed the way I introduced myself, the order and type of information I gave was mirrored by the participant. The participants kept their personal information to a superficial level inifially and this was mirrored in what the participants said of being cautious in sharing personal information with the people they work with.

My' opening statement following the introductions was; "I'd like to hear your experiences of the therapeutic relationship and boundaries in your nursing practice with adolescents - where would you like to start? ${ }^{\text {m }}$ This statement needed more clarity as three out of four participants' misconstrued boundaries as meaning limit setting as in terms: of adolescents safety, level of risk or inpatient rules. One participant responded by talking about decision making around rules in an inpatient setting, another spoke of levels of risk and limits on behaviour when moving towards discharge from an inpatient setting. Two participants were unsure of where to start and asked for more guidance. One participant spoke at length on limit setting and while connected to therapeutic relationships didn't relate directly to professional boundaries.

I was careful about the language I used as the researcher, as this could influence the direction of the narration. When I asked a participant to think of an example where she had 'set a boundary' in regard to what she was discussing at that moment in the interview, the nurse began talking about limit setting with adolescents. Asking if a boundary was set tended to move the participant away from talking about experiences: with feeling, emotion and warmth to more superficial discussion of broader issues.

Overall, the use of prompts was helpful and well utilised. The interview with the first participant made me reflect on what I could say and what I needed to question further? The participant told stories I could relate to however, as the researcher, I needed to encourage the participant to expand on the discussion to elicit practice stories by seeking other 
examples of using boundaries in practice. I was conscious of responding as the researcher rather than a nurse and very conscious of not influencing the narrative. Clandinin and Connelly (2000) note discussion between the researcher and participant is expected and part of the process when working narratively. When I sought further exampless with another participant she found this difficult. The difficulty during; the interviews was whether the issue lay with the researcher or the participant? The reflective journal I kept was useful for reflections.

The use of a reflective journal can add to the confirmability of a studily as the researcher can bring ideas to consciousness and separate these from the participants. The use of this journal provided a space for me to reflect on my influence on the participants and how or what I asked in: the interviews. Reflecting on each interview helped ensure I didn't repeat mistakes and stayed true to the narrative methodology. Reflective logs also contribute to how dependable the study is.

The participants provided rich narratives in the interviews. The practice stories where analysed utilising the three dimensional narrative iniquiry space (Clandinin \& Connelly, 2000). Universal themes transpired from the nurses' narratives. These themes are discussed using verthatim quotes from the transcripts which add to the credibility of the study.

\section{Data Analysis}

There are many ways of analysing narrative data once collectedt. The data analysis ensures a deeper understanding and exploration of the data. Polkinghorne (as cited in McCance et al, 2001, p. 355) in a personal communication states

The basic work of narrative analysis is to produce an integrated story with for each of the participants in the study. Studies using narrative analysis usually prodice a case study of each participant and then, a commentary addressing the differemces: and similarities among the case studies. 
Clandinin and Connelly (2000, p.50) who are educational researehers describe a process of data analysis called a "three dimensional namative inquiry space". This process is congruent with the philosephical underpinnings of this study as it captures the participants' experience holistically and is respectful of the nurse's experience. The analiysis is based on Dewey's notion of understanding experience and exploration of what lies beyond the experience. This is conceptualised as; both personal and social. A person's personal experience is explored asiwell as their interactions with others (Ollerenshaw \& Cresswell, 2002). Clandinin and Connelly describe this as understanding the "personalland social (interaction); past, present, and future (continuity); combined with the notion of place (situation). This set of terms creates a metapharical three dimensional narrative inquiry space, with temporality alongy one dimension, the personal and the social along a second dimensiom $\mathrm{m}_{\mathrm{r}}$ and place along a third" (p.50).

Temporality explores themes that include looking into the past, present and future within the person's narrative. This includes ideas relating; to their identity, culture, and previous experiences. The aspects firat are occurring in the present and that may occur in the future for the participant emphasises the continuity of the experience. The personal and social dimension includes looking at what is happening personally or internally for the person; her feelings and thoughts, and then exploring the social or existential factors that are present for the person the the narrative. Situation or place involves the data being analysed for specific situations that are occurring in the participant's landscape; the phiysical places of the participant's story (Ollerenshaw \& Creswell, 2002k The themes and patterns related to these three dimensions are identified for each participant's narrative.

Following this analysis I have developed an individual portrait for each nurse's narrative. The process by Clandinin and Connelly (2000) suggests the researcher should go back to the participants to gaim more material and a deeper understanding of the participant's narrative.. I 
made the decision not to do this part of the process or engage in: the process of member checking due to the evidence that suggests stories are time bound (Riessman, 1993; Sandelowski, 1993).

Member checking is the process where the researcher returns to the participants with the transcribed raw data to check that: the interpretations made by the researcher are reflective of what the participant intended to say, this process can potentially assist in adding rigour to the research (Roberts \& Taylor, 1998). Due to the befief that narratives are time bound (Sandelowski, 1993), if the participant had an opportunity to read her told narrative, she may see things differently depending on the day, and the context in which the narrative was read. This would in turn affect the quality of the data and validity of the narrative as the participants would have an opportunity to change the data (Riessman, 1993; Sandelowski, 1993). Riessman (1993, p.64) stated

A personal narrative is not meant to be read as an exact record of what happened nor is it a mirror of a world "out there", It is more about the individual's experience what happened and the effect it has had on the individual

Therefore many researchers do not return to the participants to check the data or that the interpretations made are true and accurate. this context the member checking process would potentially alter the research findings as it is thought the participants may be more concerned with getting the story right, rather than focusing the experience at the time, which is more relevant and congruent the the research. Therefore, I didn't return to the participants to check their stories, so have used a process based on Lincoln and Guba's (1985) work to ensure the study is rigorous and trustworthy, this is discussed following the data analysis.

The analysis involved developing an individual portrait for each nurse's narrative. The following tables provide a reference for the interpretation of the portraits and represent the re-storied account of each. They entail the main themes that came out of the narrations from the three 
dimensional narrative inquiry analysis and were developed using guidelines from the works of both Clandinin and Connelly (2000); and Ollerenshaw and Creswell (2002).

Table 1 includes the analysis of the data for participant one exploring each dimension of the narrative inquiry space. The personal and social dimension for this participant including themes such as; self awareness, limits on information sharing, transference and counter transference; and establishing links with the young person. Continuity looked at learning from past experiences, unresolved personal issues, learning abbout oneself and sharing of self. Situation addresses themes related to the separation of personal and professional space and the different. work settings the interactions occurred in.

Table 2 refers to the second participant's data analysis. Personall and social themes included the sharing of self with those of the same culture, the need to be cautious of sharing, role clarity, rapport building: and naming the boundary. Continuity looked at growing thought the journey with each young person, learning through past experiences, appropriate sharing. Situation discusses alternative environments for working such as a café.

Table 3 explores themes from participant three. Personalsocial identified sharing of self, naming the role, engaging, the adolescent's needs being most important and boundary crossings. Cantihuity discusses own life issues, beliefs and values, growing in awareness of self and relationships. Situation discusses visible practice in an inpatient setting.

Table 4 incorporates the data for the fourth participant. Personallsocial themes include the need for separation of personal and professiontal life, no black and while answers, self awareness and the engagement process, experiences of boundary crossings. Continuity encompasses 
life experiences, and the emphasis being on the needs of the pratient. Situation discusses transparent practice and role clarity for the nurse: 


\begin{tabular}{|c|c|c|c|c|c|}
\hline \multicolumn{2}{|c|}{ Interaction } & \multicolumn{3}{|c|}{ Continuity } & \multirow[b]{2}{*}{ Situation/Rlace } \\
\hline Personal & Social & Past & Present & Future & \\
\hline $\begin{array}{l}\text { Limited sharing of } \\
\text { personal } \\
\text { information, self } \\
\text { awareness } \\
\text { issues, } \\
\text { transference and } \\
\text { counter- } \\
\text { transference, } \\
\text { awareness of } \\
\text { own issues at } \\
\text { time of } \\
\text { interaction }\end{array}$ & $\begin{array}{l}\text { Role clarity; } \\
\text { authority and } \\
\text { friend; } \\
\text { establishing links; } \\
\text { connecting, use } \\
\text { of common } \\
\text { interests, sharing } \\
\text { of feelings is too } \\
\text { personal as are } \\
\text { issues related to } \\
\text { gender, sexuality } \\
\text { and abuse }\end{array}$ & $\begin{array}{l}\text { Previous nursing } \\
\text { experience, } \\
\text { initially rigid re } \\
\text { sharing of } \\
\text { boundaries, not } \\
\text { sharing anything } \\
\text { of self , learning } \\
\text { from experience } \\
\text { of boundaries } \\
\text { being pushed, } \\
\text { unresolved } \\
\text { personal issues } \\
\text { for the nurse }\end{array}$ & $\begin{array}{l}\text { As more } \\
\text { comfortable with } \\
\text { self able to be } \\
\text { more human and } \\
\text { real, drawing the } \\
\text { line with } \\
\text { boundaries } \\
\text { uncomfortable } \\
\text { and hard }\end{array}$ & $\begin{array}{l}\text { Sharing of self in } \\
\text { an appropriate } \\
\text { way }\end{array}$ & $\begin{array}{l}\text { Inpatient units } \\
\text { and community } \\
\text { settings, naming } \\
\text { the boundary } \\
\text { between personal } \\
\text { life and } \\
\text { professional life; } \\
\text { the need for } \\
\text { separation of this } \\
\text { both physically } \\
\text { and emotionally; } \\
\text { running into } \\
\text { patients outside } \\
\text { of work setting; } \\
\text { the need to keep } \\
\text { these interactions } \\
\text { brief }\end{array}$ \\
\hline
\end{tabular}




\begin{tabular}{|c|c|c|c|c|c|}
\hline \multicolumn{2}{|c|}{ Interaction } & \multicolumn{3}{|c|}{ Continuity } & \multirow[b]{2}{*}{ Situation/Place } \\
\hline Personal & Social & Past & Present & Future & \\
\hline $\begin{array}{l}\text { Sharing of self } \\
\text { with those of the } \\
\text { same culture and } \\
\text { ethnicity, being } \\
\text { cautious of } \\
\text { sharing re own } \\
\text { issues or } \\
\text { behaviours }\end{array}$ & $\begin{array}{l}\text { Clarity of role, } \\
\text { negotiating the } \\
\text { relationship - } \\
\text { takes time, trust, } \\
\text { rapport, visible, } \\
\text { available, } \\
\text { genuineness, } \\
\text { honesty, straight } \\
\text { up, real, humour } \\
\text { unique. } \\
\text { Friendship too } \\
\text { close, importance } \\
\text { of naming } \\
\text { boundary, using } \\
\text { humour to get out } \\
\text { of difficult } \\
\text { situation re } \\
\text { boundaries }\end{array}$ & $\begin{array}{l}\text { Learning through } \\
\text { both personal } \\
\text { and professional } \\
\text { life experience, } \\
\text { growing through } \\
\text { the journey with } \\
\text { each young } \\
\text { person, an } \\
\text { learning } \\
\text { experience from } \\
\text { sharing too much } \\
\text { with of own } \\
\text { information }\end{array}$ & $\begin{array}{l}\text { Able to share } \\
\text { appropriately, } \\
\text { importance of } \\
\text { relating and } \\
\text { sharing with } \\
\text { those of own } \\
\text { culture, own } \\
\text { gender easier to } \\
\text { share self with }\end{array}$ & $\begin{array}{l}\text { Negotiating the } \\
\text { relationship with } \\
\text { the adolescent }\end{array}$ & $\begin{array}{l}\text { Community } \\
\text { nursing } \\
\text { environment, } \\
\text { using alternative } \\
\text { settings e.g. café } \\
\text { or beach to } \\
\text { engage } \\
\text { adolescent, } \\
\text { uniqueness of } \\
\text { every individual } \\
\text { and family }\end{array}$ \\
\hline
\end{tabular}




\begin{tabular}{|c|c|c|c|c|c|}
\hline \multicolumn{2}{|c|}{ Interaction } & \multicolumn{3}{|c|}{ Continuity } & \multirow[b]{2}{*}{ Situation/place } \\
\hline Personal & Social & Past & Present & Future & \\
\hline $\begin{array}{l}\text { Sharing of self; } \\
\text { personal details } \\
\text { kept superficial } \\
\text { with limited detail, } \\
\text { awareness of } \\
\text { own issues }\end{array}$ & $\begin{array}{l}\text { Naming the nurses role } \\
\text { and the boundary, the } \\
\text { importance of being real } \\
\text { in the engagement } \\
\text { process, connections, } \\
\text { humanness, real, } \\
\text { patients needs at the } \\
\text { forefront - patients } \\
\text { needs being met, best } \\
\text { interests of the patient, } \\
\text { boundary crossings; } \\
\text { secrets, special } \\
\text { treatment, splitting. } \\
\text { Importance of clinical } \\
\text { supervision and senior } \\
\text { mentors }\end{array}$ & $\begin{array}{l}\text { Life values and } \\
\text { beliefs- not to } \\
\text { harm others, } \\
\text { awareness of } \\
\text { own issues, own } \\
\text { life story, as a } \\
\text { new graduate } \\
\text { strong emotional } \\
\text { response was } \\
\text { indicator to } \\
\text { boundary } \\
\text { crossing }\end{array}$ & $\begin{array}{l}\text { Appropriate } \\
\text { interactions } \\
\text { and } \\
\text { relationships } \\
\text { come more } \\
\text { naturally }\end{array}$ & $\begin{array}{l}\text { As } \\
\text { experience } \\
\text { grows } \\
\text { awareness of } \\
\text { self and } \\
\text { relationships } \\
\text { grows }\end{array}$ & $\begin{array}{l}\text { In-patient } \\
\text { settings ensures } \\
\text { practice is visible, } \\
\text { no contact with } \\
\text { patients post } \\
\text { discharge outside } \\
\text { of inpatient unit, } \\
\text { clarity of role of } \\
\text { the nurse - } \\
\text { powerful position }\end{array}$ \\
\hline
\end{tabular}




\begin{tabular}{|c|c|c|c|c|c|}
\hline \multicolumn{2}{|c|}{ Interaction } & \multicolumn{3}{|c|}{ Continuity } & \multirow[b]{2}{*}{ Situation/Place } \\
\hline Personal & Social & Past & Present & Future & \\
\hline $\begin{array}{l}\text { Personal and } \\
\text { professional life } \\
\text { separation, } \\
\text { boundaries } \\
\text { individual for } \\
\text { each nurse, no } \\
\text { black and white } \\
\text { answers, } \\
\text { Importance of } \\
\text { clinical } \\
\text { supervision and } \\
\text { senior staff to } \\
\text { learn from, } \\
\text { importance of } \\
\text { self awareness, } \\
\text { nurses own } \\
\text { issues }\end{array}$ & $\begin{array}{l}\text { Engagement process } \\
\text { important; realness, } \\
\text { understanding, } \\
\text { empathetic, not a friend, } \\
\text { who's needs are being } \\
\text { met? Patient is the } \\
\text { focus, overstepping of } \\
\text { boundaries can repeat } \\
\text { trauma for adolescent, } \\
\text { boundary crossings; } \\
\text { secrets, buying of gifts. } \\
\text { Sharing of self in a } \\
\text { limited way, emphasis on } \\
\text { patients needs }\end{array}$ & $\begin{array}{l}\text { Nurses own } \\
\text { issues and life } \\
\text { experiences, all } \\
\text { have experienced } \\
\text { hard times }\end{array}$ & $\begin{array}{l}\text { Nurses } \\
\text { personal life } \\
\text { is kept } \\
\text { separate }\end{array}$ & $\begin{array}{l}\text { Emphasis on } \\
\text { the patients } \\
\text { needs }\end{array}$ & $\begin{array}{l}\text { Inpatient practice } \\
\text { visible boundary } \\
\text { crossings, } \\
\text { transparent } \\
\text { practice, clarity of } \\
\text { the role of the } \\
\text { nurse-a } \\
\text { professional one }\end{array}$ \\
\hline
\end{tabular}


Representing these side by side I explored universal ideas and qualities of the narrative. While considering the parts I have kept in mind the whole of each narrative. Clandinin \& Connelly (2000, p.145) state

Part of the narrative inquirer's doubts come from the understanding that they need to write about people, places and things as becoming rather than being. Their task is not so much to say that people, places, and things are this way or that way but that they have a narrative history and are moving forward. The narrative research: text is fundamentally a temporal text - about what has been, what is now, and what is becaming.

The ideas from each portrait were brought together to create three distinct universal themes which evolved through this process. Thiese themes correlate with the three dimensions of the analysis process and allinterrelate.

I have entitled the theme related to temporality that has transpiredi from the participant's narratives a learning journey. The sub-themes fromit the murses ${ }^{i}$ narratives that arose to create this theme include:

- personal life values and beliefs

- learning through personal and professional life experience

- growing as a nurse throughout the 'journey' with the young penson

- the nurse's awareness of own issues especially unresolved issues

- the culture of the nurse. More sharing with adolescents of the same ethnicity or gender

- a new graduate nurse being 'closed' in sharing about self, this changing as experience and understanding grows, becoming 'more human and real' in the relationship

- learning from experiences where boundaries are 'pushedl' or "crossed"

- as experience grows self awareness and relationships grow

These sub-themes relate to the nurses' experiences of the past, present and future in the nurse's personal and professional life. 
I have named the main overarching theme from the personal and social dimension of the analysis keeping the adolescent and the nurse safe. The sub-themes include:

- engaging the adolescent - establishing links and connecting ing the relationship

- the nursing skills of engagement; honesty, realness, availability, genuineness, empathy and humour

- the sharing of self by the nurse

- ensuring the sharing meets the needs of the adolescent, keeping the nurse and adolescent safe, "whose needs are being mell?"

- keep personal and professional lives separate

- naming the role of the nurse

- naming the boundary

- experiencing own boundary crossings and being witness to athers

- transference and counter transference

- 'splitting' special treatment and 'secrets'

- parallel processes

- is the interaction in the "best interests of the patient?"

- clinical supervision

- senior nurses' as mentors

These sub-themes cannot be seen as separate from each other $r_{1 \text { rather }}$ they all interrelate and overlap as do the dimensions of the three dimensional inquiry space. Sub-themes related to temporality also cross over to relate to the personal and social dimension and with the place or situation.

The broad theme titled clarifying of the role of the nurse relates to the nurse's role within the relationship and links with place or situation in the narrative inquiry space. The sub-themes of this theme include:

- naming of the nurses role

- a nurse not a friend

- power differential within the relationship 
- naming the boundaries

- separation physically and emotionally of the nurse's personall and professional life

- hope for the adolescent and his family

- uniqueness of every adolescent

- the visibility or transparency of the nurses practice

- contact with adolescents post discharge

- meeting outside the practice setting

- community and in-patient settings

- alternatives to clinical environments to aid engagement

The use of the three dimensional narrative inquiry space has allowed a hrolistic representation of the nurses' narratives.

Clandinin, et al. (2006, p. 22) found

such a metaphoric space would help them represent not only the wholeness of their vives and the lives of the participants with whom they engaged but would them engage in the unfodding experiences that have temporal, personal, social and place aspects

As shown in the analysis of the nurses' narratives each dimension is interrelated and overlaps. This format for data analysis ensures the wholeness of the narratives is respected and the nurse as a person is represented. Trustworthiness and rigour are important processes in. all research. The following section looks at the significance of ensuring the research process followed in this project is a rigorous one.

\section{Determining Rigour}

Determining rigour in qualitative research involves ensuring the successive steps in the research have been carried out clearly. This is important as it ensures methodological accuracy and that the research is a worthy piece of work (Roberts \& Taylor, 1998). These steps: are defined in this section drawing on the works of Lincoln and Guba (1985).

There is much debate as to the most suitable ways of determining rigour in qualitative research (Sandelowski, 1993; Schneider, et al 2003). Many researchers have differing opinions on what constitutes an appropriate 
criteria (Clandinin \& Connelly, 1990; Koch, 1998; Lincoln \& Guba, 1985; Morse \& Field 1995; Overcash, 2004; Riessman,1993; Sandelawski, 1991). When we think about rigour in relation to the constructivist world view there is not one correct reality, rather, multiple realties exist The culture and experience of both the researcher and participants play an important part in the outcome of the research. Therefore no two prieces of research of this type will be exactly alike. The interpretative nature of narrative inquiry also supports this view. The application of strict rules about what determines whether a narrative account of experience is a truthful one is an ongoing debate (Sandelowski, 1993).

The criteria for determining rigor vary. Some include looking at issues such as reflexivity, rapport, and coherence (Roberts \& Taylor, 1999). It is necessary for the researcher to determine an appropriate method that is congruent with the methodological assumptions of the research. Lincoln and Guba (1985) have identified four criteria for determining rigour and trustworthiness in qualitative research. These inictivde: credibility, transferability, dependability, and confirmability. The use of these criteria over others has been chosen for this project due to Limcoln and Guba's method being supported by Sandelowski, a noted narative nurse researcher, and its relevance to this project.

\section{Credibility}

Credibility refers to whether or not the data is a true and accusrate account of the experience narrated by the participant. Sandelowski (1986, p.30) states "a qualitative study is credible when it presents such faithful descriptions or interpretations of a human experience that the people having that experience would immediately recognise it from those descriptions or interpretations as their own". The use of verbatim quotes from participants' interviews and transcripts, are a means of determining credibility. The grammar in quotes may not necessarily be correct as they are transcribed as they were spoken. Verbatim quotes are used in the findings chapter of this thesis. 


\section{Transferability}

Transferability indicates the research results are able to be generalized or transferred to the wider population. Due to the small sample sizes and the contextual nature of many qualitative research projects it is often debated whether or not the results are representative of the wider population or only the specific population sampled (Overcash ${ }_{\mathrm{r}}$ 2004). However, it is argued that with narrative research, it is the nursing theory which is interpreted from the results (Morse \& Field, 1995). The theory may then be generalised and thus applied to other settings. It is the job of the researcher and the reader of the research to decide whether or not the results can be transferred and are applicable in a particular setting. This would depend on a variety of factors including whether or mot the mature of the interactions were similar to that described by the participants in an alternative setting (Morse \& Field, 1995). The results of research demonstrate that it is possible to transfer theory as: the findings are discussed in other publications.

\section{Dependability}

Dependability or reliability in research assumes that the method car: be replicated to achieve the same results. This is more manageable in quantitative research. However, in qualitative research, the empkasis is often on the uniqueness of human experiences, therefore reliability is mere difficult to demonstrate. Lincoln and Guba (1985) discuss use of audit to address dependability. This could be achieved by following the outlined research process in a project to check congruence and! gain an understanding of the logic used in the process (Sandelowski $i_{x}$ ig.86). Dependability is shown in this project through the description of the research process and its relevance in terms of congruency from: the theoretical underpinnings to the method used in the research process:

\section{Confirmability}


Confirmability of a project ensures the researcher's biases have been stated and are separate to that of the participants' subjective experience. Confirmability can be determined when the other three criteria have been met. The use of a reflexive journal is also helpful in determining whether or not a study is confirmable as it provides an opportunity for the researcher to document and bring to consciousness his own wiews (Lincoln \& Guba, 1985). I have kept a journal throughout the research process; writing my own ideas and reflections prior to the interviews with the participants and documenting my reflections of the interviews and processes after the interviews. It was valuable for me as the researcher to keep a certain distance from the participants and to be sure of my role as the researcher to contribute to the confirmability of the project.

When looking for trustworthiness in narrative research, it is important to acknowledge there are many views, no one reality, and that there is not one path for processes to follow or one set of formal rules. Sandelowski (1993, p.8) states

we can preserve or kill the spirit of qualitative work; we can soften our notion of nigor to include the playfulness, soulfulness, imagination, and technique we associate with more artistic endeavours, or we can further harden it by the uncritical application of rules

There is always potential for the researcher to become preoccupied with ensuring the correct procedures for the research are followed. Whille this is important, it can potentially take the focus away from what the research is about - the participants' experiences. However, there are specific guidelines that do need to be followed to ensure the research process is easily understood and there are rationales for decisions made by the researcher. It is important that the research processes are congruent throughout the study.

\section{Conclusion}

The process and considerations undertaken to obtain nurses' experiences of therapeutic boundaries has been explained. This includes the research plan, theoretical underpinnings of narrative inquiry, 
ethical considerations, data processes and rigour. In thennext chapter findings of the participant's narratives are discussed. 


\section{CHAPTER 5}

\section{Findings}

The main aim of the study was to identify nurses' experience of assessing, understanding and working with therapeutic boundaries when working alongside adolescents in mental health. The objectives of the research were

- To understand through nurses' practice stories their experience of developing the therapeutic relationship with adolescents.

- To explore how nurses might make sense of therapeutic boundaries in these relationships and how they negotiate these with the adolescent.

- To determine what factors influence nurse's thoughts and ideas around the defining of therapeutic boundaries

Using Clandinin and Connelly's (2000) three dimensional namrative inquiry analysis three main themes were identified from the murses' narratives. This analysis process has enabled a holistic view the participants' narrative which is reflected in the findings. The themes include; clarifying the nurse's role, a learning journey and keepung: the adolescent and the nurse safe. These themes have been explained in this chapter, included is examples of the raw data narrated by the murse participants.

\section{Theme 1: Clarifying the nurse's role}

The theme was identified from the analysis process that addressesplace and situation in the three dimensional narrative inquiry space. This refers to the setting in which the stories take place. The setting is a broader concept than just the physical environment; it encompasses: the milieu in which the relationship between the nurse and adolescent develops. Defining the role of the nurse within this milieu was an 
essential first step for the participants in exploring therapeutic boundaries.

Clarifying the role of the nurse within a community setting of in an inpatient setting is a theme that was significant for all the participants. The complexity of this was discussed in their practice stories: The participants told stories of the need to first engage the adolescents; they paid particular attention to the roles culture and diversity play in the engagement process. They related issues of the power diffential present in the relationship and the need for the relationship be adolescent focused. The nurses shared specifically how and the nurse is able to effectively share something of herself within the relationship and named the difficulties in maintaining both the therapeutic relationship, and professional boundaries which are beneficial both parties.

The participants all acknowledged the significance of engaging the adolescent as the first step to forming a therapeutic relationstipt. The nurses felt being available, visible, honest, 'straight up', warm, caring, empathetic, trustworthy, genuine, and human contributed the formation of this relationship. They each told their story of the challenges of engagement and how making a connection with the adolescent and his: family involves the nurse using a variety of skinss.

I would say probably the use of humour, being real, building a relationship, being there, all that stuff yes it is the same, but your approach to the young person is different because you need take them for who they are.

Forming links and connecting with a young person and her family is essential in the engagement process. Through this process the murse and adolescent may identify people or places in common or aultural experiences they can relate to. One participant talked about her experience engaging with young people of the same ethnicity to her: 
You know with the ethnicity thing ... I might share a little bit more around my experiences with a young Tongan than I would a different stimicity because we relate a bit more. We understand the culture a bit more and the dynamics that they face.

She also acknowledged the importance of not assuming that the same ethnicity or culture may assist in the engagement process;

it is unrealistic to say that all Pacific Islanders work well with all Pacific Islanders because some ... don't want to work with other Pacific Isfanders and within the Pacific Island culture there are sub cullimes of that ... Island.

She described subcultures including different religious beliefs;, New Zealand born or Island born Pacific people. Having knowiedge of different cultural practices and beliefs, enables the nurse to wank with these differences when engaging adolescent;

Yes, just in regards to Pacific Islanders, you know um one-on-one therapy can be really something that is quite distress[ing] to a young person because it is not normally done. You know they don't normatly sit with their parents one to one and talk about their lives.

The importance of treating adolescents as individuals, with each having different needs and therefore requiring a genuine individual approach was noted. One participant said;

I find that working with the concept of culture and diversity keally important and you cannot generalise what you do with young people because if you ... do ... they will know. ... they won't want to be sartiof it because they all want to be special (ha ha) you know. 
She also stated that adolescents are 'very astute' and can pick up; when a nurse is not available or genuine in the interaction.

The participants emphasised the need for the nurse to have boundaries around the amount she shares about her personal life with the adolescent. Conversely, the participants all thought it was important to share something of themselves when engaging the adolescent. Getting the balance right and not sharing too much of their personal lives; with adolescents was challenging.

I do not disclose a lot of personal things but I am able to show a young person that I am a human being as well. That I have feelings, and I do have the ability to understand within a context where I don't necessarily disclose a lot about myself.

The emphasis for the participant was on sharing something of herself that related to the young person's experience; therefore the sharing had a clear intention

If it is relating to the young persons experience I would share and I would do that because that is the connection, that is part of the rapport building and the forming of trust. If it does not relate to the young person I won't share it.

As a rule of thumb for me ... if I think a family is having difficulty engaging I will use anything as long as it's not too personal. It is usually about interests rather than ... sharing feelings too much. It is more about ... sharing interests perhaps like talk about music. Music is always good to talk to teenagers about.

Shared interests helped the nurses to establish links with the adolescent; and this required conscious use of boundaries as the adolescent could easily misinterpret this sharing as one nurse explained; 
Often I have ... talked about things that I do within my personall life, leisure time for example, in a way as ... establishing a link with a feenager ... I was working with one boy in ... and I mentioned to him that Is every year in ... I used to go to a festival ... he ah actually wanfed to come with me ... at that time I obviously realised that it had gone too far and I had to kind of close it down but he didn't like it, he didn't know why, and it hurt having to draw the line but I guess I knew then that self disclosure is fine but is the actual sense when it starts to end the therapeutic relationship.

This nurse recognised the importance of being 'self aware' when the relationship had gone too far and was able to name this with the adolescent even though it felt difficult and made the murse uncomfortable, and no doubt the adolescent too.

Two participants mentioned taking into account a young person's gender; the different gender of an adolescent played a part in the amount the nurse was willing to share about herself with that young person

Well I mean obviously the gender thing. Males are different from females. Just in regards to personal information, I wouldn't really share a lot with males that I would a female in regards to their own experiences and that is just commonsense I think.

The participants spoke of the importance of clarifying the role when engaging in a relationship with the adolescent. When the nurse has the ability to be up front and name the nurse's role explicitly with the adolescents, this was found to be helpful in ensuring there were appropriate boundaries within the relationship.

I won't keep secrets, that we can have a good time but at the same time you know I am a nurse and that is what I am being paid to do, that is what I am here to do and I am willing to care but by no means will $k$ have a close friendship. 
Tiwo participants provided a rationale for why it's important to clarify the role for the young person and the confusion it creates if a relationship outside of the realms of a professional one is allowed to develop.

As a nurse on the unit I am very aware that I am very powerful ta them and if I have a relationship out of the unit then I think it would become very messy ... If they had to reenter the unit again then the relationship would be changed and it would be different.

If don't want to kind of create that relationship outside work as well. ldon't think that is really conducive to a professional relationship as; well particularly when my family's involved. I was with my son one day/ and this teenage boy came up with this mobile phone and I had been working with him when I was an intake worker. He ... said "Oh ... how are you? is this your son?"I felt he was encroaching so um I kind of just said, "yes: it is," pleasantly and then moved on ... I knew I just want to keep my family life separate.

The role of the nurse for the participants is a professional one that requires clear and consistent boundaries between the nurse's personal and professional life. This concept of professionalism and the need to keep boundaries can be hard work but is not without warmth and caring as the following nurse described:

I give them care and understanding. I really try not to be judgmental although sometimes I can ... It is quite hard in a way to have boundaries, to keep those boundaries in place when you care for them, you know eare what is happening for them and not sort of go overboard. Ithink one of the things that I have always asked myself right throughout my nursing is what I am doing is it good for the patient?

The setting of boundaries and limits in the engagement process with the adolescent can be difficult as it has the potential to create discomfort for 
both the nurse and the adolescent and may risk the adolescent disengaging.

At times in the past I have had to state specifically with teenagers: that I am the adult nurse and they are the patient.

Another nurse found the use of humour useful when limit setting was needed;

I would say "I don't want to get into that ... It is not something that is appropriate for us to talk about especially when you have a problemw:. But also humour is very important ... it buffers a lot of things as well.

One participant spoke of times when it was better to name the difficulty and admit to not being able to engage an adolescent for the benefit of the nurse and the adolescent.

I have had patients that I cannot build a therapeutic relationship with and I have ... handed them over to somebody else. ... I know there are patients that I don't particularly like. However I still endeavour to dib my nursing best for them, these are the ones I work hardest on.

Maintaining a therapeutic relationship and boundaries with adolescents is a complex task. The nurse needs to be clear about her role the adolescent; the participants in this study found that naming role explicitly was helpful. The need to treat young people as individual's; with differing needs was also emphasised. The nurse having the ability to share something of herself with the adolescent was significant not without its own challenges. Being able to name the difficulties with the adolescent often helped in ensuring the relationship remained therapeutic. 


\section{Theme 2: A learning journey}

The learning journey, a phrase used by a participant, was the theme identified from the analysis process focusing on temporality (past, present and future). The nurses in this research told stories of their own life experience $e_{r}$ and the effect on their role as a nurse: They spoke specifically of the need to be aware of their own issues from their: life stories and the effect these can have on relationships and therapeutic boundaries with adolescents. The nurses told stories of their experiences of self disclosure and the changes in disclosing personal information as their practice progressed.

Each nurse talked about the effect her own life story has on her mursing practice. This includes not only events in her life, but also her xalues, beliefs and culture. One spoke specifically about the morals and values ingrained in her by her parents;

I suppose it is in a way part of my life, is because my parents targhtt... us not to do harm to others ... also animals and to the environment, and they went on and on about that sort of thing. So it has ... been right throughout my life not to do harm to others.

The nurse spoke of carrying these values and beliefs on in her life and her role as a nurse caring for adolescents experiencing mental iliness:

The participants spoke of adverse events in their lives which tradi an effect on who they are as people, and nurses. One nurse spoke about unfinished business in her life in relation to being sexually abused. She talked about the importance of being aware of this unfinished business and unresolved personal issues, when working with young people with similar experiences She related a time when working with an adolescent, when this issue came up for her. It created a strong emotional response which she says was unusual for her and was a signal that she needed to be careful in her role as a nurse; 
I could see this little girl, I could see myself in her at her age being terribly confused and you know, distressed, but not wanting to her parents about what was happening. It was... like I see myself in a way.

When asked whether this response was unusual the nurse replied

Yeah well it is now, it wasn't when I was younger. I would blow my stack when I was younger. And I did see that because I saw myseff in this young person so much that it was all ... unfinished business stuff ass well.

This nurse was able to recognise, with the help of a colleague, the potential for a boundary crossing in this situation. Her strong emational response made her realise the potential to confuse what had happened to her, with the experience of the young person she was working with:

Another nurse noted the potential for transference to occur when working with adolescents whose gender differs from that of the nursen. The participant noted recognition and acknowledgement of the transference and counter-transference within the nurse-adolescent relationship is valuable for both the nurse and the adolescent and when acknowiged, can aid in the development of the relationship.

The participants all talked about being 'human', which they related as having their own life story which affects their experiences with young people and families. One described the way that she often joins with, or empa thises with a young person who is experiencing distresss is to share that ... we have all had hard times within our lives. Two aurses who commented on their own hard times said they don't disclose fo the adolescent what these 'hard times' are as this may take the focus; away from the adolescent and what is happening for him at that time.

I have never ever disclosed to a young person that I was sally abused as well, but I sometimes will say that I understand how they are 
feeling because I had experienced something similar ... I ... pick whether it is really necessary or not.

Disclosing the fact that I have had ... I would say I was sick as a childlibut I wouldn't go into detail and other young people saying oh that is so sad that you have had ... knowledge of that just feels too close ... that's my family stuff.

The participants felt it was important to not share anything too persanal or too intimate about their personal lives. Two described a techrique of asking themselves whose needs is this meeting? When sharing information about themselves, is your actions in the best interest of the patient or if it isn't what is happening here? They felt if it was not related to what the adolescent was experiencing then it shouldn't be strared. One gave this reason for not sharing personal information;

"I think that they are here to be dealing with their own stuff. Althoughiwe can say ... we have been through tough stuff or that we are human beings ... at the same time presenting your own issues or them kmowing about worries that other staff have, ... puts more pressure on themiand it might take more responsibility away from them.

A nurse related at times sharing with young people that she is having a bad day. The nurse described this as being real for the benefic of the adolescent. She felt this was modeling to the young peoples that everyone can have bad days and we all need to find ways to manage these. However all the participants talked of being cautious of disclosing their feelings to adolescents. This was in response to ensuring the emphasis of care was not taken off the adolescent and the risk of the nurse's issues becoming the focus.

The nurses described how as new graduate nurses, they were very rigid in relation to boundaries: 
I think when I first started in nursing I was very, very clear in my mind that I would not self disclose I was almost too kind of closed and I think over the years ... I was not quite relating to the teenagers I was working with and that is pretty important.

All the participants experienced a time early in their nursing practice where a crossing over of a boundary or a potential boundary crossing could have occurred with a patient.

You know I gave her my cell phone ... which was a work cell phone ....and said ring any time. So she would ring me at 3 in the morning.

I can remember back in my early years you know when you shared a little bit too much and then you'd get stuck because the therapeutic relationship is no longer therapeutic... They see you as a friend and you are too close.

The nurses acknowledged the role these experiences played in their learning as a nurse and the influence on their practice;

But that was quite a good lesson for me too, I mean I really learnt from that, that although I could be caring and ... look after them and things like that, that my own experiences could come in to it and I needed to be really aware of that.

There is still a relationship there because we journeyed together but that is an example of ... me growing as a young health worker.

The participants recognised the role experiences such as these play in their practice today. As they grew as people and had more selfawareness this in turn has an effect on the relationship with the adolescents. When the nurse is more self-aware, she feels safer about sharing more of herself in the relationship; 
I think ... I have improved along the years. I have gained a lot of insight about myself as well and it is about my practice ... so it has been a learning journey.

Over the years I have been prepared to kind of reveal a little bit more of myself. Just appear a little bit more real.

When talking about her nursing practice one nurse participant described;

I have sort of like evolved ..., through experience, I have ... been wbte to refine [my practice]... It has come more naturally to me.

The impact the nurses" past, present and future has on relationships: with adolescents is significant. When the nurse is aware of this effect, she is able to use her knowledge and experience to advance the relationship, while ensuring the needs of the adolescent is the focus the relationship.

\section{Theme 3: Keeping the nurse and the adolescent safe}

The previous themes note the need for the nurse to be aware the role her own life issues can play in relationships with adolescents. This third theme, which relates to the personal and social dimension of the narrative inquiry space, explores this further. When the nurse is straring herself with the adolescent and engaging in this relationship;, the participants emphasised the need for the nurse to protect the adolescent who is often vulnerable. The nurse also needs to protect herseff. By sharing herself with the adolescent the nurse can leave herself vulnerable.

This theme initially explores boundary crossings described by the nurses in their practice stories. The participants' related being witness: to boundary crossings in the practice of their colleagues. It must be noted 
these events are perception of the participants. These were mainly related to practices witnessed by inpatient nurses and this may be because practice is more visible in an inpatient setting. Nurses perceptions of how some relationships with adolescents appear to be meeting the needs of the nurse rather than the adolescent are also explored. The participants shared strategies for monitoring this risk that contribute to keeping themselves and therefore the adolescent safe.

The nurses in this research are aware of the varying opinions an what constitutes a therapeutic boundary and a boundary crossing. One murse said,

For me [it is] the ability to care and share myself but within a limitedl way. This might sound critical but I always believe that I have very good boundaries and that I have seen a lack of boundaries with my colleagues' practice. I think that people find it difficult to challenge because it is such an individual thing and there are certain limitations.

This nurse and another identified incidents they perceived as boundary crossings by their colleagues.

Taking someone to their home ... I mean, things that seem obvious but happen. Buying ... presents. I think if it is a birthday then it should be a unit thing ... The same with Christmas and all those sorts of things. Giving a phone number, your address, sending mail and making ptione calls post discharge. And other staff members mentioning health problems or whatever to certain young people. [Having] coffes: and taking them to appointments and driving them around post discharge, or treating [the patient] as a staff member, disclosing information about other patients. [The] same applies with families ... the disclosures that are given to them that is about other patients or staff in situations: like that I think the same boundaries apply within the family. 
The participants talked about wider issues that occur as a result of this behaviour by nurses. They viewed nurses asking adolescents to keep secrets had the potential to lead to more serious boundary crossings. Sharing a practice story about secrets one nurse explained

Yes I think, definitely not okay, that often these young people are brought up in the environment where we don't talk about things or we will keep things a secret which generally leads to an admission [to hospitial]] or more trauma. And so for a nurse to say, 'Oh look I bought you thisis for your birthday but don't tell anyone, and for me to go and say, 'oh that's a nice necklace where did you get it?' and for them to say, 'oh I cantit tell yous.

This participant made the link between the young person's previous experience of secrets, which at times lead to abusive situations, and this being reenacted by the nurse asking the adolescent to keep mare secrets. This is often described as a parallel process when the behaviour in the therapeutic relationship mimics that of the patient's life:

Constantly being bought gifts but within a secret environment of "Bon't tell anyone but I got this when I was in ...' or I got you this.... The difficulty l think is other ... staff or nurses being able to perceive that they are actually doing more harm than good, I think, and other nurses kind of realizing that this person was quite attached to them and their ability; to pull back a wee bit.

When discussing boundary crossings these nurses described using a strategy to monitor their own practice. They would ask themselves whose need is this meeting? They shared their understandings af what these boundary crossings may be about;

I think it's a professional trying to make themselves feel better about this person's trauma. But by no means is it a quick fix. And we are an acute unit it's a short stay. This person is used to being abandoned and lthink 
it just draws them in to that attachment thing. Whereas, if you have clear boundaries around the nurse-patient relationship that I am here: for support, but I am not a friend, and I am someone that you can takk tond I can give a neutral opinion and I can listen.

The participants talked about using some kind of self disclosure in their work with adolescents but didn't see this as a crossing of boundaries rather it was useful in connecting with the young person. However, they all had parameters around this and articulated a rationale for sharing.

Self disclosure is a way of kind of breaking down boundaries I thin that is all that is needed as long as it is not happening as an angoing therapeutic tool. I think if it felt as if I was being counselled the teenager then I would realise it had gone too far.

Because families and young people just get so anxious about ...t this is never going to change, this is it, this is as good as it gets, ... one of my roles I find is that if you can bring hope to a family that is going through that with their young person that is the best thing you can do and for me that story of the journey with my best friend because it is evidence.

If it is relating to the young person's experience I would share and I would do that because that is the connection. That is part of the rapport building and the forming of trust. If it does not relate to the young person I won't share it.

The nurse-adolescent relationship and maintenance of therapeutic boundaries is very complex and is recognised by the participants. Three of the four emphasised the importance of clinical supervision in their practice. Clinical supervision provides the nurse with a space to reflect on practice to ensure it is safe and therapeutic. One nurse said was important to check things out in relation to her practice And that practice issues to supervision was another way of keeping herself safe in her work. She said, I can't live without it actually, and also used 
supervision to check out the nursing practice she saw from others: grior to challenging some of this. Another participant said,

The only way I started to get my boundaries was I insisted on getting some supervision and when I was sharing these things in supervision they were giving me some advice and giving me any suggestions on how to try things.

The participants also talked about the importance of learning from senior colleagues and mentors and having these people available to help keep her safe in practice

[There] was me as a young community support worker, new in the area and at that stage I did not actually have a mentor or somebody to actually say oh ... you know. Or provide that supervision to say .... bok at your boundaries, maybe next time give her some times that she carining you between, you know, those kinds of things.

One nurse found as a new graduate getting it right in relation to professional boundaries was challenging, I asked the nurse about how she came to her understanding about therapeutic boundaries

.... very difficult and I think it has been a professional development thing that I have found for myself, often looking towards senior colleaguessand asking them about advice and also kind of a personal ethical judgement looking at other people's practice and disagreeing with certain aspects that I have seen ... The ultimate question comes up in supervision ... whose needs am I meeting by buying this person presents or whatever the issue may be.

Another participant talked about her role as a mentor and senicar staff member in helping to keep others safe in their practice 
If I notice somebody else, like a mentor or support worker, if I motice them stepping over the boundaries a little, I will pull them aside and talk to them.

One participant spoke of strategies to keep herself safe when wvorking with adolescents

I would step back a little bit ... as though I was on the outside looking at the situation and constantly thinking in my head ... Is this ethical? .... So we managed to get a relationship that was ethical and therapeutic.

Another described her conscience doing this for her:

I recall one time when I had promised this person a king sized of chocolate if they were able to manage through a therapy session, I remember dwelling on that for like the whole night, not being able to sleep and then going to one of the senior staff and saying, look, if feel like I have just bribed this person and I had a real hard time about it"... I think it does not feel right within yourself.

What is right for one nurse may in fact be a boundary crossing for another. The complexity for nurses in maintaining boundaries that are therapeutic is shown in the narratives. The need for nurses to monitor their practice in keeping themselves safe, but also having opporiunities to discuss and reflect on this with others is significant. Whether in informal discussions with senior colleagues or mentors, or in elinical supervision.

As previously stated the themes from the analysis cannot be seen in isolation. They encompass a holistic perspective from the interrelationship with each other. The need for the nurse to claxify her role and maintain therapeutic boundaries is critical to ensuring both: the adolescent and the nurse are protected in the relationship. 


\section{Conclusion}

The themes from this research are complex and enlightening. The concepts and issues in the themes are interrelated. The formation af the therapeutic relationship was significant for the participants and being honest and upfront with the adolescent helps the relationship develop. Showing warmth, caring and an interest in the young person enables: the nurse to connect with the adolescent.

The data suggests the nurses' life experiences and personality playj a significant role in forming relationships and maintaining therapeutic boundaries. As the nurse's experience and awareness of herself have grown, so has her ability to connect with, and form effective relationships. Having an awareness of her own issues and an ability to reflect on these and the part these may play in the relationsh with adolescents is crucial. The participants found some self disclissure useful in forming therapeutic relationships with the adolescents but it needs to be related to what is happening for the adolescent and the benefit of the adolescent.

Significant learning came from their own, and others experiences; of "boundary crossings". Learning from senior colleagues and mentoss: was important in ensuring boundaries were therapeutic. Three participants found clinical supervision played a significant role in keeping the murse safe in her practice.

Although there are challenges in discussing and disclosing such personal and complex issues as professional boundaries the participants provided rich, valuable practice stories. They have shared their experiences openly in the hope of contributing to the quality of cares the adolescents receive. 
The next chapter discusses the findings further linking them to rellevant literature. The implications for nursing practice, nursing education and further research are also explored. 


\section{CHAPTER 6}

\section{Discussion and Recommendations}

\section{Introduction}

The themes elicited from the nurses' experiences of professional boundaries are complex and multilayered. From the practice stonies; the participants have raised pertinent issues for further discussion. it has been essential for me as the researcher not to lose sight of the whole person when analysing the data and collating the findings. Clandinin et al., (2006, p.22) emphasised this importance when they talked about representing "people not as taken apart by analytic categories but: as people who were composing lives - lives composed over time full of richness and complexity". When the whole person is kept in mind this ensures a true representation of the persons narrative.

This chapter is a further extensive explanation of the themes of this research. The findings have been compared to relevant available literature in this field. I have particularly drawn on the works of Rene Geanellos, an adolescent mental health nurse who completed a Doctorate on "Explicating Practice Knowledge of Adolescent Mental Health Nursing". Her work is extensive and inspirational in exploring: the role of the adolescent mental health nurse in an in-patient setting. Whave utilised nursing theory specifically from Geanellos' work as well as relevant literature from both adult mental health nursing and paediatric nursing to inform this discussion. Hildegard Peplau's work: on interpersonal relations in nursing has been drawn on including the use of psychodynamic concepts. Psychodynamic nursing theory provides a theoretical framework to gain a better understanding of some of the experiences of the participants practice stories. The implications for nursing adolescents in mental health are discussed. The limitations of 
this study have been outlined. Possible recommendations for mursing education, nursing practice and nursing research are explored.

The themes all interrelate and shouldn't be seen as being separate; from each other. 'Clarifying the nurse's role' looks at both place and sitwation and the formation of the relationship with the adolescent. 'A learning journey' explores issues related to past, present and future life experiences and nursing practice. 'Keeping the adolescent and' the nurse safe' enables an exploration of issues related to the potential vulnerability of not only the adolescent but also the nurse. It a a exploration of the effect that personal and social issues have on the nurse's ability to assess, understand and work with therapeutic boundaries with an adolescent in a mental health context.

\section{Clarifying the nurse's role}

Engaging the patient in a therapeutic relationship is considered central to the role of the mental health nurse (Geanellos, 1997; Moylew 2003; Murray \& Wright, 2006; O'Brien, 1999). The participants in this study found to build rapport the nurse needed to use a variety of skills. It is important for the nurse to be available and visible to the adolescent to form the relationship. Being available, for the participants in this study, meant the nurse needed to have a genuine interest in the young person, and be present in the interaction with the adolescent. The nurses; felt this genuine interest was shown by the nurse's ability to be honest and straight up which means not having any secrets or unspoken mintives within the relationship. Showing warmth, caring, and empathy the adolescent was also emphasised. So although the nurses fielt a straight up attitude was effective in engaging the adolescent it needed to be portrayed in a thoughtful and therapeutic manner.

When adolescents are in the stage of cognitive development where they are able to experience formal operational thought the ability think about what others may be thinking is not yet clear. Therefore if murses 
are not open and honest with the young person in the relationship the adolescent may be suspicious of the nurse and her intentions. Because of the adolescents developmental phase this is magnified for the young person, therefore engagement may be made more difficult.

Participants in this study shared the need to go out of their way when aftempting to engage an adolescent. This often meant going to a cafë or somewhere that was more neutral territory for the young person. Here the nurse consciously does things with the young person that differs to the nurse's usual practice in an attempt to engage the adolescent and help him feel comfortable. This was not seen by the nurses as a crossing over of boundaries as it was discussed as being for particular purpose - engaging the adolescent and was a planned conscious intervention, with a clear intention behind it. When nurses have clear intentions of what they are trying to achieve in the relationship with the adolescent, boundaries are able to have some flexibility. However this jurdgment is made more easily when the nurse has sufficient elinical experience to base these decisions on. Being more flexible when attempting to engage the adolescent has the potential to give a mixed message, which may lead to the adolescent pushing the boundlaries further and/or not understanding the reason or intention behind the murse's flexibility. Practice that differs from the norm often needs to be discussed explicitly with the adolescent to avoid confusion.

The participants felt it was important to be a person the adolescent could trust, felt was genuine in her interactions, and was interested in the adolescent. This contributed to engaging the adolescent and ultimately the formation of a therapeutic relationship. Being human and real im the relationship was also emphasised. This is supported in the literature: by both Geanellos (2002) and Scanlon (2006). Geanellos (1997 pi20) describes having "a genuine love of adolescents" which aids in engaging adolescent and forming a therapeutic relationship with young people (Murray \& Wright, 2006). 
The sharing of self was discussed by all of the nurse participants in this study as being essential to engaging the adolescent in a therapeutic relationship. When deciding how much or what to share the nurse participants were consistent that the importance of sharing personal information was for the benefit of connecting with the adolescent in a therapeutic relationship.

This sharing was usually about common interests or activities. Geanellos (1997, p.29) supports this as she found in her experience "sharing contextually appropriate information about myself and my work with other adolescents reduces the distance between myself and the adolescent". However, this sharing has the potential to be pushed too far. The adolescent is searching for his identity, and is looking for role models to help base this on. Therefore he may question the nurse to share more than she feels comfortable with or more than is appropriate. In my experience this boundary pushing is an everyday occurrence in interactions with a young person. However, sharing helps to reduce the adolescent's feelings of isolation and feelings he is not alone in his experience and that there is hope for recovery.

Within a nurse-adolescent relationship an inevitable. power differential exists. This was acknowledged by one nurse participant who described finding it helpful to name this when clarifying the role of the nurse. When nurses and adolescents are struggling for power this can act as a diversion from core issues and also lead to mutual mistrust, which challenges the therapeutic nature of the relationship (Peternelj-Taylor, 2002; Ramjan, 2004). The relationship can then mirror what the adolescent may have had with other authority figures such as parents. The adolescent is separating from his parents in an attempt to form his own identity; he creates conflict to allow this to occur. As with the adolescent's relationships with parents, the relationship with the nurse needs to be negotiated. Clear boundaries and discussions around the nurse's role and what could be negotiated within this relationship can be helpful. The importance of compromises and negotiation when working 
alongside the adolescents was illustrated in the nurse participants' practice stories.

The role of the nurse needs to be one of partnership with the addrescent however, while negotiation, trust, and the "sharing of power are important" there needs to be no doubt of the adult's role in the relationship, and it is essential the nurse is the "professional/adult in charge" (Geanellos, 1997, p.29).

This trusting and negotiating can cause tensions within the relationship with adolescents. These tensions occur for a variety of reasons; young people are attempting to gain a sense of autonomy, therefore, having nurses as well as, or in the place of parents, giving advice, setting, limits and boundaries can be a challenge for the adolescent. Unfortunately there is insufficient evidence or research on how these relationshiss may improve (Ramjan, 2004). The nurses in this study felt that naming; the difficulty or tension with the adolescent was helpful are you annoyed with me? One nurse felt this could be uncomfortable for the nurse and the adolescent; therefore, talking about it was a step towards resolwing the difficulties. This corresponds with the nurse's opinion on the importance of being honest or straight up when engaging the adolescent.

One nurse felt it was important to acknowledge when the relatidnship wasn't working and to request assistance or a change of nurse when this appeared unable to be resolved. Geanellos (1997, p.264) identifies the need for "the team" to step in at times and allow the nurse to take some time out or have a break from challenging adolescents. Acknowiedging this is important (Higgins \& McDougall, 2006) although this may: be difficult as the nurse may feel some kind of inadequacy related this. Nurse participants in this study talked about adolescents being very astute, therefore they will also be aware of the difficulty the nurse is having. When the nurse is honest about what she may be experiencing with the adolescent this can be addressed in a helpful way. 
Working alongside adolescents in a partnership role was as essential in forming and maintaining relationships with adolescentsi. The ability for the nurse to compromise, negotiate and admit when thixgs are not going well is important. When adolescents can contribate in negotiating the relationship with the nurse, care can be individualised to meet their needs.

The need to individualise the treatment and care adolescents receive was found by the nurse participants as an important aspect when engaging the young person in the therapeutic relationship. Adolescents are exploring their identity, therefore being treated as individualis; who have differing needs can aide in validating who they are as young people.

This nurse highlighted the importance of a young person of a particular ethnicity or culture would not necessarily want to be treated like others of that ethnicity. The value in not assuming we have an understanding of a persons culture because of their ethnicity is recognised as important with other cultures in New Zealand. Durie $(1995$, p.9) supports this when he found "far from being homogenous Maori individuals have a variefily of cultural characteristics and live in a number of cultural and socioeconomic realities. The relevance of so-called traditional values is not the same for all Maori". This highlights the importance of findirm out from individuals how they wish to be treated in terms of their culkure and individual needs.

The drive for mental health consumers to be treated as individuals: with differing wants and needs has largely stemmed from the corssumer movement (Walsh, 2002). This movement has enabled minority groups to be more visible and the cultural and other specific needs of these groups to be identified. In New Zealand it is recognised that mental health nurses need to respond to their clients in ways that recognise their individuality (O'Brien, 1999). I believe processes such as mihimaihi or introductions allow for people to make connections with each other. 
Beliefs and values could begin to be addressed, which is significant in engaging the adolescent and his family.

Walsh (1997) discusses the importance of nurses in all areas of nursing being able to care effectively for patients of all cultural backgraunds. This is especially important with adolescents who are exploring their identity (Erikson, 1968). To be able to provide culturally safe mursing care; where the adolescent feels his cultural needs are respected, nurses need to be acutely aware of their own culture, to recognise the differences in others and accommodate those differences. Cultural safety was not discussed explicitly with the participants in this study, although a participant acknowledged how her boundaries may differ when working with an adolescent of her own culture. The else: of mihimihi at the beginning of the interview process allowed the murses space to acknowledge their own culture as part of the research process. Ramsden (2000, p.4) stated

Cultural safety should be the experience of all recipients of nursing care. It is about protecting people from nurses, from our culture as health professionals, our attitudes, our power and how we manage these things whether unintentionally or otherwis.

The importance of a secure identity and self awareness in adolescent mental health nursing is essential. Gastmans $(1998, p .6)$ states: "If: the individuality of the patient is not properly recognized nurses run the risk of reducing him or her to a mere function of themselves or to a means towards their own self-development". When the nurse is aware of her own culture and identity this can be role modeled for the adolescent. When nurses become more confident in who they are including their own culture and having an awareness of their own issues, they can share more of themselves appropriately within the relationship withi the adolescent. This sharing can be helpful but nurses need to have: some understanding about the role of this sharing (Geanellos, 1997).

The role of a child and adolescent mental health nurse is not well defined (Armstrong, 2006) but is one that can be particularly diverse. As 
described by a nurse participant the role requires the nurse wear many caps. Including being an authority and friendly. Because of this diversity the nurse doesn't always get it right.

When attempting to engage an adolescent in the therapeutic relationship there are many aspects to consider. The nurses participating in this research discussed the need for the nurse to be genuine, up frant and honest with the young person. The sharing of self as it relates contextually to the adolescents' experiences was significant. Wanking alongside the adolescent while attempting to instill hope wasi also important. The need to recognise individuality and acknowledge cultural differences was essential.

The nurse participants told practice stories of becoming more comfortable and more open with young people as their nursing experience and knowledge about themselves developed. This affected the quality of the adolescent-nurse relationship. The following theme explores this.

\section{A learning journey}

The participants in this study all told stories about how their life experience influenced who they are today in relation to their personal life, but more specifically who they are as nurses. Both their personall and professional experiences have contributed to how the nurses practise when working with adolescents in mental health. As they know themselves better, and became more self aware of their own issues, this provided opportunities to work more effectively with adolescents experiencing mental illness. This section explores how murse participants feel their practice has changed since starting out. Boundary crossings or near boundary crossings have played an integral role in the nurses learning. 
The nurses in this study have had four to 15 years experience: in adolescent mental health. As nurses' experience grows the nurse matures, and is able to draw on past experiences to enrich their practice today (Peternelj-Taylor, 2002).

One nurse participant said initially she was very cautious about what she shared with patients about herself. The nurse described perhaps being "too rigid" and closed with her patients, to the point where she didn't reveal much about herself at all. In my clinical experience it is often easier for a beginning nurse to be cautious or rigid on the amount she would share of herself as this is a way of protecting herself as a: new practitioner from the potential of doing the wrong thing or inifiating a boundary crossing with the adolescent. The beginning nurse will follow guidelines which are often rigid as they cannot cover individual situations or be applied to every individual in the same way (Benner, 1984). When the nurse is more experienced she is able to integrate her experiences of practice and move around the guidelines to become more flexible in her mursing practice (Benner, 1984).

Adolescents searching for their sense of identity will push the nurse to share about herself as they are often searching for role models; or an opportunity to experiment with their opinions. Even when the nurse is consciously cautious or closed in sharing about herself the adolescent will continue to push this boundary. As the nurse participant deseribed, being rigid or closed made her less human, and therefore less accessible to the adolescents she was working with. Being more real enablles the nurse to engage more effectively with the adolescent and plays an essential part in forming the therapeutic relationship. This realness is a consistent theme throughout the participants' narratives.

All of the nurse participants in this study described an experience where they were responsible for an interaction that could have led to or been considered a boundary crossing. These were experiences the nurses were able to learn from. They are not regarded as mistakes, not nursing 
effectively or as being unimportant. Ord (1998, p.43) found "the capacity to acknowledge, discuss and learn from our emotional reactions is vital if we are to create environments in which troubled people and those: who care for them, can feel sufficiently held and supported". These early experiences help shape the experiences that follow, with the nurse learning from these previous interactions. As Dewey $(1938$, p.47) said "every experience should do something to prepare a person fart later experiences of a deeper and more expansive quality. That is very meaning of growth, continuity, reconstruction of experience".

The nurses all related that these occurrences were valuable learning experiences and had a beneficial role in helping shape their future nursing practice. Hawes (2005, p.17) acknowledges that a a haugh learning from our mistakes is challenging the "getting it wrong" helps nurses to learn how to "get it right". In nursing a lot of learning cames from the nurse making mistakes and doesn't get the interpensonal boundary quite right (Totka, 1996). As one nurse participant described a boundary crossing was an example of me growing as a young health worker. Growing, learning and ongoing development is continuous in the field of nursing (Cutcliffe,1997; Geanellos, 1997). A nurse participant spoke of this learning when she described evolving through her experience as a nurse.

Geanellos (1997, p.16) states "knowledge is not singular, instlar or unchanging rather it is shaped by traditions of the past present and, in turn, will shape the future. This is a dynamic representation of knowledge". Practice knowledge in nursing is described as ever changing (Geanellos 1997), the nurse's experiences within her personal life, family and culture and professional life all shape the knowledge that she contributes as the role of an adolescent mental health nurse. The journey of learning is continuous journey for the nurse throughowet her nursing career. 
The nurse participants narrated openly their early practice had in shaping their current and future nursing practice. Having opportunities to reflect and feel supported in their practice was invaluable to them. The following section explores ways the nurses did this while looking further at some of the challenges they may face in relation to ensuring they/ have therapeutic boundaries with adolescents.

\section{Keeping the adolescent and the nurse safe}

The themes identified from the nurses narratives all interrelate, with the murse's personality a strong thread in each theme. The nurse's personality and ability to demonstrate self awareness plays a significant role in ensuring the adolescent and nurse remain safe. This section explores the nurse's role in protecting the adolescent. The kurse participants' interpretation of boundary crossings or potential boundary crossings is discussed. Some of the principles of psychodymamic mursing have been explained and discussed as an interpretation of why some these boundary crossings may occur. The connection between psychodynamic nursing and undergraduate nursing education New Zealand is explored, along with some key strategies in prowiding apportunities for the nurse to ensure her practice is safe.

Psychodynamic principles are important concepts in mental health nursing (Peplau, 1952). Peplau acknowledges the significance these principles play in helping nurses to improve their interpersonal relations with their patients. "Being able to understand one's own behaviour; to help others to identify felt difficulties and to apply principles of human relations to the problems that arise at all levels of experience - these:are functions of psychodynamic nursing" (p.xiii). Working with adolescents experiencing mental health issues is complex (Geanellos, 1997; Ramjan, 2004). The use of self or the nurse's personality plays a major role in the work mental health nurses do (Peplau, 1952) therefore there is putiential for boundary crossings to occur. Many boundary crossings were discussed by the nurse participants in this research. Two nurse 
participants spoke of psychodynamic concepts. A nurse participant noted that when nurses are sharing more of themselves it is easy to lose sight of psychodynamic issues such as transference that may be occurring in the nurse adolescent relationship.

Transference and counter transference are psychodynamic comcepts. Experiences and feelings that occurred in the past, are experiencedias if they were in the present (Casement, 1985). Recognising transference and counter transference can be complex, and the nurse needs have some understanding and self awareness, otherwise there is a nisk of blaming others for strong emotional responses which belong to the nurse not the patient (Obholzer \& Zagier Roberts, 1994). When murse recognises her own issues and how they may effect work she does; with a patient, it can be very beneficial to the relationship. This was illustrated by another nurse participant when she described having a strong emotional response to a young person who had been sexually asused, as she had, as a child.

The nurse was able to recognise herself in the young girl which could be related to unfinished business in her life story. Casement (1985, 7) acknowledges how difficult this can be to recognise, or make sense of, as "what comes from whom, in any two-person relationship is nol allways clear. This is because the processes of communication can be either projective (one person putting into the other) or introjective (one person taking in from the other)". The nurse's strong emotional response was a signal to her that the interaction required some reflection.

This nurse clearly had the skills, with the support of a colleaque, to recognise transference in the relationship, and that it had come frum her past experiences. Therefore she needed to be careful and seek support from her colleagues in reflecting on her practice. Because af this unfinished business the nurse was experiencing a strong entational response, which if not recognised could lead to the nurse blaming the adolescent therefore confusing the issues the adolescent is experiencing 
further. This nurse who is New Zealand educated, didn't name: this specifically as transference. This could have been elicited via a second interview or one could also suggest that the nurse may not haveen aware of this theoretical framework. Although she did recognise that there was a connection to her life story, taking this a step further to mame it as transference wasn't done.

Many adolescents I have worked alongside are very vulnerable. They have often had occurrences where they have experienced tramma or their sense of self (which is still being developed) has been damaged in some way. Nurses want to care for and support these distressed young people in the best way they can. Even when nurses have the kest of intentions, decisions or interventions are not always in the best interests of the adolescent. A nurse participant in this study described how she found it challenging ensuring she kept her professional boundaries.

The nurse participants witnessed many incidents in the nurse adolescent relationship where they felt boundaries were crossed. The following section explores why some of these incidents may occur utilising; the psychodynamic concepts of mirroring and projection identification. These concepts help to increase our understanding of this complex area.

Projective identification is a concept often recognised in therapeutic inpatient settings. Halton (1994, p.16) described projective identification as "an unconscious inter-personal interaction in which the recipients; of a projection react to it in such a way that their own feelings are affected: they unconsciously identify with the projected feelings". If the projection of the adolescent's feelings is not recognised by the nurse, this could also lead to projective identification.

A participant told the practice story of a young girl who had been sexually abused, the nursing team specifically assigned to look after the adolescent became entrenched in the relationship with her, the young person often had difficult times where she would accuse the nurses of 
not caring about her, when one nurse responded with "if I didn't case: why would I buy you chocolate".

If we look at this process the patient may be projecting feelings into the nurse of needing to feel special, or feel loved. These feelings may have been previously responded to by the adolescent being bought gitts by adults that may be abusing her. The adolescent projects this needinto the nurse, the nurse responds by buying the adolescent presentss as a way of acknowledging the young person is accepted or special. As the nurse participant recognised, this could mirror behaviour the adolescent has previously experienced. The incident of the nurse buynigg: an adolescent chocolate could be a boundary crossing, or it could be considered every day practice. The boundary crossing wouldn't necessarily be the act of buying the chocolate, but the intention befind it, or what message it was portraying to the young person. If the nurse was doing this to make the young person feel special or to like her more, this could be considered the boundary crossing. However, this could allse be a way of engaging the adolescent. The boundary crossing occurred when the nurse chose to use this example of buying the chocalate to communicate to her that she cared. This could be very confusing for the adolescent who is already confused about whether or not being bought gifts means an adult cares. It appears the nurse received a projection of feelings from the adolescent; a mirroring has occurred between the nurse's response and the response the adolescent is used to getting from someone abusing her.

The adolescent may have also been projecting feelings into the murse, this may have lead to a projective identification with the murse responding as though the feelings belong to her. Mirroring; and projective identification are common terms in psychodynamic nursing. They help to provide an understanding of the complexity of interpersonal relations in nursing (Peplau, 1952). 
When relaying various practice stories that involved boundary crossings, it was acknowledged the nurses involved were responding the patient's communication. The nurse's responses to these communications were not consciously meant to further traumatise the adolescent.

This nurse participant felt this incident of gift buying was not necessarily driven by the nurse, it is the nurses role to reflect on incidents such: as this when they occur and get some support in managing it. When the nurse is not able to do this, the cycle is not broken and therefore the adolescent doesn't learn alternative ways of getting his needs met within relationships.

These dynamics which lead to boundary crossings can also create another psychodynamic process known as "splitting". Splitting is; the mame of the process when feelings from one person are dividedi into differentiated elements (Halton 1994), or in this case nurses.

One nurse participant had an incident where an adolescent was bought presents by some staff members for her birthday. The nurses bought these presents with their own money. Some nurses didn't feel this, was appropriate. This was noted by the adolescent who displayed derogatory behaviour toward the nurses who didn't buy her a present. This split was created by the nurses and then exploited by the adolescent. The feelings of the adolescent in the nurse's practice story are divided into; "good nurse" who gives a young person a present forthis birthday, and "bad nurse" who doesn't. The adolescent put his feelings that he finds difficult to bear into one nurse (the nurse who doesnit buy presents) and the good feelings that can be tolerated into another murse (the nurse who buys the presents). Splitting that is allowed to occur in an inpatient setting can make it extremely difficult for adolescents to be nursed effectively. When nurses are split into the "good" and "bad" nurse this can result in increased tension and distress within the nursing team. This has an effect on her ability to care for the adolescent. Parents: are 
often put in positions of being the "good" and the "bad" parent. If parents or nurses teams don't discuss this dynamic the young person cam feel very powerful by exploiting these splits, which lead to keeping the adults in these positions. Moreover detrimental when an adolescent cannat see the "good" and the "bad" which can be integrated into one person.

These psychodynamic concepts transference, counter transference, projective identification and splitting are complex and often difficull for a nurse to recognise. When not recognised and discussed with support of a team they can create boundary crossings which lead to challenging behaviours acted out by the adolescent. Peplau (1952, p.71) when discussing the complexity of interpersonal relations in nursing, states;

Needs, frustration, conflict, and anxiety all provide energy that is transformed intom some form of action. Understanding what these experiences are, which compel desifuctive or constructive responses from nurses and patients, is a step in the direction of personal and rational control of behaviour.

It is the nurse's role in the relationship to develop an understanding of these behaviours for the benefit of the adolescent (Geanellos, 1997)

The amount of boundary crossings the nurse participants had been witness to in this research was surprising. This is understood as relating to the fact that two of the nurse participants were inpatient murses therefore the nurses' practice is much more visible. The nurses are all working alongside the same adolescents which makes nursing practice transparent and open to discussion with others. This in turn proxides opportunities for other nursing staff to witness potential boundary crossings, and also provide valuable opportunities for support and discussion with these issues.

Another possible interpretation for the amount of boundary crossings could be related to a wider issue of nurse undergraduate education in New Zealand. The nurse who spoke specifically of transference and counter transference was educated in England. A New Zealand nurse spoke about the psychodynamic concept splitting, and although all of the 
nurse participants could describe boundary crossings and recognise these were inappropriate they didn't take this further to discuss a theoretical framework behind these nursing interventions. This could be a result of the methodological design of this research with only one interview with the participant this was not elicited or as being related to a gap in undergraduate nursing education in New Zealand.

\section{McMahon and Ward (1998, p.35) state}

Learning to tune into the transference and to be aware of one's own countertransference takes time and practice and it tends to be learned or within a training context, so that there will be an opportunity to reflect on possible meanings and connections without jumping to conclusions or - still worse - imposing these am the child.

In a New Zealand study, Peters (2003) looked at the contribution of mental health education in undergraduate courses. This study included the undergraduate nursing degree. She found nurses could have little as two weeks mental health theory and 160 hours of mental health clinical practice, with one programme delivering one hour education on adolescent mental health issues. This was also supported by the KRM Strategic Review of Undergraduate Nursing Education (2001) who found that the current undergraduate programme for nurses in New Zealand doesn't address mental health issues appropriately (p.74). Peters: also comments on the curriculum taught, in her report there is no mention of psychodynamic principles or concepts. Therefore, with little on no education on these concepts in their undergraduate nurse educatian; it is more difficult for nurses to recognise and name psychodynamic cancepts such as transference and counter transference. The education around these concepts is reliant on learning from the practice of others.

Griffiths and Leach (1998) found the use of psychoanalytical ideas by the nursing profession in the United Kingdom has been limited, atthough these concepts and ideas are used in the United Kingdom in therapeutic community settings (Griffiths \& Leach, 1998). The nurse participant in my study who named transference as something to be aware of in terms of managing her professional boundaries was educated in the United 
Kingdom and worked in a therapeutic community setting. my experience of working in England, psychodynamic nursing theory was used extensively in child and adolescent inpatient settings.

Psychodynamic nursing theory can be used to inform, guide and influence practice in adolescent mental health nursing. Peplau (1952, p.xi) states "nursing is helpful when both the patient and the nurse grow as a result of the learning that occurs in the nursing situation". She argues the ability for "recognising, clarifying and buildingy an understanding of what happens when a nurse relates herself helpfiully to a patient are the important aspects in psychodynamic nursing".

Geanellos (1997, p.33) found "the nurses ability to work therapeutically with adolescents, and collaboratively within a team, are influenced by herself and her capacity to work through unresolved personal issues". These psychodynamic concepts assist in providing the nurse with a better understanding of how her personality and life experiences can have an effect on the relationship with an adolescent, which will benefit the adolescent.

When nurses haven't had undergraduate education in psychodynamic mursing they need to learn about these experiences through working in an environment where other nurses have had this experience. Open communication about these experiences from other nurses helps: the nurse to learn about these psychodynamic concepts through expexience in their relationships. The inpatient environment where practice is more visible also contributes to allowing this to occur. Opportunities for murses to discuss the challenges interpersonal relationships with adolescents create incorporating psychodynamic concepts, help in keeping boundary crossings to a minimum.

The question asked by the nurse participants "whose needs this meeting" is an effective way for the nurse to monitor her own practice in 
ensuring potential boundary crossings don't occur. Another of ensuring safe practice is clinical supervision.

Clinical supervision was discussed by three of the four participantss as essential in helping to ensure the mental health nurse is practicing safely. Barker (1998, p.67) highlights the importance of elinical supervision for mental health nurses when he said "supervision in psychiatric nursing has two aims: to protect people in care from nurses and to protect nurses from themselves". When nurses reflect in clinical supervision on their practice with the adolescents this prowides opportunities to explore and work through some of the unconscious pracesses that may be occurring in the relationship indluding psychodynamic concepts. Geanellos (1997, p.22) notes the significance of reflection when she said

The nurse juggles these aspects of her relationship with the adolescent by refectimg, on her interactions with and responses to the adolescent's thoughts, feelings: and behaviours. In this process she comes to understand more deeply, aspects of trer:own adilescence, her relationship to her parents, authority figures and herseff. She confronts unresolved emotions, her primary way of operating in the world, knowledge defieits, assumptions and belief systems. She receives the opportunity for learning from the adolescent with whom she works and from the milieu in which she finds; fierself

The nurses utilised clinical supervision and discussions with senior nurses and/or other colleagues as opportunities for reflection and leaming. These spaces also provide an opportunity to process same of the interactions with the adolescents.

Roberts (1994, p.118) discusses the importance of those in helping professions having insight into the reasons for choosing the particular tine of work. She acknowledges personal therapy can be helpfull in achieving this "to disentangle one's past from the present, and find alternative ways of resolving unconscious conflicts, rather than needing to do this entirely through one's work". When a nurse has kad: an opportunity to work through and resolve her own issues, this ensuress she is more available to the adolescent and can use those life experiences appropriately to engage, empathize and exchange experiences with the 
adolescent (Geanellos, 1997). Roger (1980, p.148) states "the more psychologically mature and integrated the therapist is, the more helpful is the relationship that he or she provides. This puts a heavy demand on the therapist as a person". The need for the nurse to look after herself well in other aspects of life have a sense of hope and positive ouflook assists in sustaining her in this complex area of work (Geanellos, 1997).

The management of therapeutic boundaries with adolescents in mental health nursing is complex. It is necessary when exploring such an issue to keep in mind the whole person when analysing and discussing data from research participants. The need to clarify the role of the murse when engaging an adolescent in the therapeutic relationship helps to ensure the boundaries within that relationship are determined from the beginning. Being explicit and naming this for the young person was helpful. Nursing practice changes as the nurse's experience develops. The nurse becomes comfortable with herself as a person as she grows and develops, and her awareness of her own issues develops. The nurse's personality and her ability to share some of herself with the adolescent were necessary in developing therapeutic relationships. When sharing information about herself the nurse needs to be aware of the potential crossing of therapeutic boundaries.

These themes identified by the nurse participants provide insight into how these nurses assess, manage and explore the complex area of therapeutic boundaries when working with adolescents in a mental health setting. For nurses to be able to make sense of therapeutic boundaries in relationships with adolescents they need to have an understanding of theoretical frameworks such as psychodynamic nursing. Psychodynamic nursing principles need to be taught in undergraduate and post graduate nursing programmes, ongoing discussions and education around these principles need to be everyday practice for nurses to be able to recognise and process the dynamics occurring when working with such a complex age group. Clinical supervision and open discussions with senior staff and mentors also 
provide thinking spaces where the nurse can monitor her professional boundaries within her nursing practice.

\section{Implications for adolescent mental health nursing}

Adolescent Mental Health Nursing is a specialised area of practice. Although a lot of findings in this research could be generalised to other fields of nursing, there are specific implications in regards to therapeutic boundaries for nursing practice for Adolescent Mental Health Nurses. This section summarises some of these complexities.

As this thesis has revealed, the developmental stage of adolescence is complex. The adolescent's cognitions, body, and emotions are in constant change. The adolescent is searching for a sense of identity: All of these issues contribute to the adolescent's vulnerability. This: has implications for nurses working alongside the adolescent who is coping with normal adolescent issues as well as experiencing mental iliness. Through the search for an identity the adolescent is separating from that of his parents. For this separation to occur conflict is created the parent adolescent relationship. Although he creates conflict, the still requires much support and involvement. A danger of this relationship is the adolescent may idealise the nurse, a helping adult, because of this conflict with his parents. This idealisation of the nurse can make her feel special to the vulnerable adolescent. Therefore, the nurse tries harder to help the adolescent risking a blurring of her role and the crossing of professional boundaries. Another contributing factor is if the nurse has unresolved issues related to her adolescence or life experiences which can add to the complexity of the relationship. Self awareness is essential for the nurse to be clear about her role with the young person as this can impact on the vulnerability of both involved.

The adolescent's personality is still developing. The search for: an identity requires the adolescent to have role models whom he can base his identity on. The nurse is often a role model to the adolescent. The adolescent will push the nurse on a daily basis to share information 
about herself and do more than her nursing role. These are every; day occurrences for adolescent mental health nurses. For an inexperienced nurse managing adolescents pushing boundaries, boundary crossings can occur before the nurse becomes aware of it. Actions, interventions and even conversations with adolescents need to be planned, thoughtful and have clear intentions. So how does the adolescent mentall health nurse manage this and ensure she is sincere and genuine in her interactions with the adolescent? The concept of professional boundaries needs to be at the forefront of the nurse's mind at all times to ensure she can respond in a therapeutic and professional manner-

This research revealed that without sufficient knowledge of the fundamental principles of adolescent mental health nursing; sureth as knowledge and skills in both adolescent development and psychodynamic nursing, nurses are at risk of boundary crossings. Adolescent mental health nursing is a specialist area of pxactice; therefore nurses need to have sufficient education and experience. Nurses practicing in this field in New Zealand need to obtaim post graduate education in this specialty. One way of achieving this is through the generic courses. However this doesn't address the specific role nurses have in working psychodynamically. This needsi to be addressed in undergraduate and post graduate nursing progranmes. Another way of achieving this education would be in exchange programmes abroad, where there are established adolescent mental health units such as the units where I was employed in England.

For adolescent mental health nurses to make a difference they need to have a clear understanding of the developmental stage of adolescence, the effect this has on the nurse's role and psychodynamic principles. The adolescent's state of turmoil and change requires support of adults with understanding. The nurse needs to be clear about her role within the relationship and take responsibility to ensure safety and bourndaries are therapeutic for the adolescent's benefit. 


\section{Limitations}

This sfudy has provided valuable insights into some of the challenges facing nurses when assessing, monitoring and maintaining therapeutic boundaries with adolescents in mental health. Due to the limited published research found in adolescent mental health nursing: it is difficult to come to sound conclusions. The methodological design of this research and time constraints have also had a potential impact on the depth of the stories told and the eliciting of the participants understanding of the narrations. This study involved four murses' experiences of this complex issue.

From this research it is clear there are no definitive answers related to how nurses assess, monitor and maintain therapeutic boundaries; with adolescents in mental health. As one participant narrated:

it is an issue that is not black and white and the grey areas are individual for other people.

However, the results of this study have raised some interesting questions in relation to the complex issue of therapeutic relationshipsi and professional boundaries. These recommendations need ta be considered within the wider picture as they are developed from ai very small sample in New Zealand. Further research is required to be able to generalise the findings into the wider population. And perhaps comparative research with my first work experience in England would benefit both New Zealand and English nurses. However, the findings of this research do provide possible considerations for future mursing education, nursing practice and nursing research.

\section{Recommendations}

The findings of this research provide an insight into possible interventions that would be beneficial for nurses in helping to maintain therapeutic boundaries with adolescents experiencing mental health 
problems. Outlined are possible recommendations for munsing education, nursing practice and nursing research.

Undergraduate education in New Zealand has a very limited mental health component (Peters, 2003; KPMG Consulting, Health, Education and Community Services, 2001). The focus appears to be mare: on diagnosis, management of risk and cultural issues. Although these topics are important in our risk adverse world, the focus has beent taken away from the importance of psychodynamic nursing. Learning about these concepts would benefit nurses in understanding the significance of therapeutic boundaries and provide nurses with a theoretical framork to understand the complexity of the relationships with adolescents. The therapeutic relationship is the forefront of mental health nursing (Peplau 1952).

Nurses are still being employed into mental health services New Zealand directly from their comprehensive training, although thene are new graduate programmes available (Walsh, 2002) which provide murses with greater understanding of the complexities of mental health nursing. The broader picture of these knowledge deficits and limited mental health education has an effect on recruitment and retention of mental health nurses in New Zealand (Walsh, 2002). Nationally all murses should be employed through Nursing Entry to Practice Progranmmes. These programmes have adolescent streams which would address some of the issues pertinent to adolescent mental health needs. However, adolescent mental health nurses still need to undertake specific post graduate adolescent mental health education to be able to care for adolescents and maintain therapeutic boundaries effectively. Funded training schemes such as exchange programmes for adolescent mental health nurses to countries which have established psychodynamic nursing practices would be invaluable to New Zealand mirses' development. 
For murses already working in this complex area, this study has identified the need for nurses to have a clear understanding of their own issues. These issues may have an impact on their relationships withi the adolescents with whom they are working. Nurses would benefit from having forums to discuss some of the challenges boundary crassings and psychodynamic nursing create. The nurses in this study emphasised the importance of clinical supervision and learning from more experienced nurses in helping to keep their practice safe. Because boundaries differ for each nurse, a culture where nurses feel safes and supported to have open discussions around boundaries both informally and in formal arenas would be helpful. These experiences would assist in providing nurses with learning opportunities and would support a culture of transparent practice supporting nurses in keeping themselives and adolescents safe.

Further research is needed into this topic and adolescent mental health nursing. This research could focus on the formation of the therapeutic relationship with adolescents experiencing mental health problensi. It could look further at the specific area of therapeutic boundaries; from both the nurse's experience and that of the adolescent. A larger study with a bigger sample size would enable a more in-depth exploration of these issues already discussed. The cultural differences and inflivences of working in New Zealand also require further exploration and research. These areas are pertinent to adolescent mental health nursing: and require further published research to provide evidence based nursing in this challenging but rewarding field.

The findings of this research provide some clear recommendations which could have possible implications and recommendations for mursing education, nursing practice and further research in the area: of adolescent mental health nursing. However, because of the limitations of this small study this would require further exploration to contibute further to adolescent mental health nursing in New Zealand. 


\section{Conclusion}

This study has provided some valuable insights into some of the challenges adolescent mental health nurses face when assessing, understanding and managing therapeutic boundaries when working with adolescents experiencing mental health problems. There are no clear answers on how boundaries are managed. Boundaries may differ for individual adolescents and on different occasions with the same adolescents. The nurse's boundaries are challenged everyday in clinical practice with adolescents.

The nurses in this study found the first step to ensuring therapeutic boundaries in relationships with adolescents was to clarify their rofe in the relationship. This includes engaging the adolescent. This engagement process involves the nurse sharing something of herself with the adolescent. It is the nurse's responsibility to ensure the amount she shares with the adolescent doesn't allow the relationship to move from a professional to a personal one and that sharing is of benefit to the adolescent.

The nurse grows and develops in her clinical practice with adolescents. She becomes more aware of ensuring her boundaries are therapeutic. As the nurse learns more about herself this learning benefits her relationships with adolescents. The nurse learns from her experiences, being either too closed or too open with the adolescent. This leaming journey enables the nurse to consolidate her knowledge of the challenges in working alongside adolescents.

Managing therapeutic boundaries is not easily done as this research has identified. The need for the nurse to take responsibility in keeping both herself and the adolescent safe within the relationship is paramount. Strategies to help the nurse do this include; having an understanding of psychodynamic nursing as a theoretical framework to understand the relationship and the role boundaries play, and receiving support from others through clinical supervision, open discussions and support from 
senior nurses. Adolescents are at a unique developmental stage that challenges thase working with them. The nurse needs to have boundaries at the forefront of her mind at all times when working with this complex age group. Adolescents are challenging but rewarding and fun individuals who have a lot to contribute to nurses' knowledge and learning.

This research has confirmed what I have seen in my nursing practice; managing therapeutic boundaries is a challenge for nurses. Without sufficient education and experience to manage these issues, nurses are putting themselves and the adolescent at risk. Writing this thesis and completing this research has been an invaluable experience for me both personally and professionally. Revisiting the Tavistock Clinic and the adolescent inpatient units in London and Oxford would provide me with an opportunity to share this research and further enhance my skills. A sabbatical in England would enable me to explore the possibility of setting up exchange programmes for nurses working in this specialty in the United Kingdom and New Zealand. The exchange of knowledge in practice can and does shape our lives. My experience of working in this specialty in England and participating in post graduate education has shaped who $\mathrm{I}$ am as a person and an adolescent mental health nurse. New Zealand adolescent mental health units need to have a culture: which focuses on psychodynamic nursing practices as this will ensure beneficial outcomes for adolescents experiencing mental illness and their families.

Finally I would like to sincerely thank the nurse participants in this study. These four people have provided rich and valuable stories of their experiences of working with adolescents in this complex specialty. 


\section{Appendix 1}

\section{Human ethics approval}

TE WHARE WĀNANGA O TE ŨPOKO O TE IKA A MĀUI

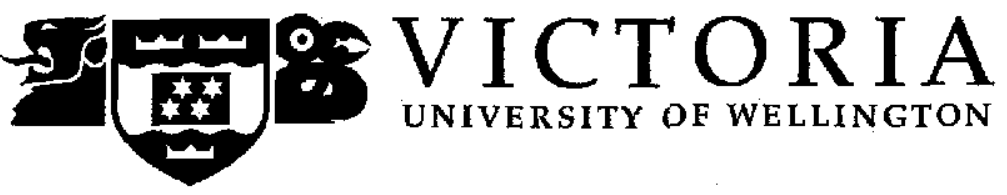

\section{MEMORANDUM}

$0-4-4635676$

0-4-463 5209

Allison.kirkman@wuw.ac.nz

\begin{tabular}{l|l}
\hline TO & Julia McLean \\
\hline COPY TO & Thelma Puckey \\
\hline FROM & Dr Allison Kirkman, Convener, Human Ethics Conmittee \\
\hline DATE & 16 December 2006 \\
\hline PAGES & 1 \\
\hline SUBJECT & $\begin{array}{l}\text { Ethics Approval: No 144/2006, Pushing the boundaries: } \\
\text { relationships with adolescents. }\end{array}$ \\
\hline
\end{tabular}

Thank you for your application for ethical approval, which has now been consideredi by the Standing Committee of the Human Ethics Committee.

Your application has been approved and this approval continues until 1 November: 2007. If your data collection is not completed by this date you should apply to the Human Ethics Committee for an extension to this approval.

Best wishes with the research.

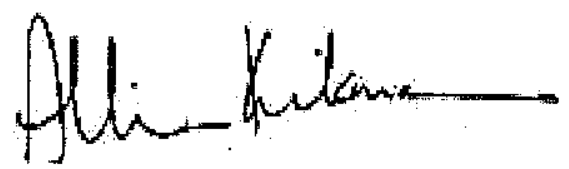

Allison Kirkman

Convener 


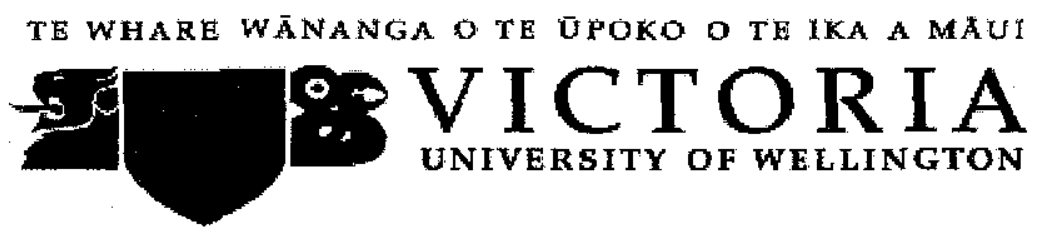

\section{Appendix 2}

\section{Participant information sheet}

Pushing the Boundaries: Relationships with Adolescents

\section{INFORMATION SHEET FOR PARTICIPANTS}

My name is Julia McLean I am currently employed by Taranaki District Health Board as a Community Mental Health Nurse working with children and adolescents. As part of my Masters Programme at Victoria University I am looking to complete a small research project for which I would like to invite your participation. The research has ethics aproval from Victoria University Ethics committee and will follow their recommended guidelines.

My study aims to explore how nurses manage professional boundaries with adolescents in mental health. There is currently limited research in this area to assist in the defining of boundaries within this setting.

I plan to interview a small number of Registered Nurses employed in District Health Boards, who are currently working in adolescent mental health. Using a narrative design I am seeking nurses' descriptians of their experience of defining boundaries with adolescents in mental health. I will use unstructured, in-depth interviews face to of approximately 1-2 hours duration, at a time and place convenient you.

Every effort will be made to ensure the participant's identity remains confidential. However, due to the small number of adolescent mental health nurses in New Zealand, and the narrative design of the research, there is a possibility that participant's views/experiences may be recognised by their peers. No identifying features will be used in the final report. The names and geographical location of the participants will be kept confidential at all times. The interviews will be audio taped, transcribed and then analysed. The transcriber will sign a confidentiality form. My supervisor, the transcriber and I will be the only people have access to the transcripts. Participants will have the right to witidraw from the research up until two weeks after the interview at whictit time none of their data will be used.

The information obtained from the research will be written up in the form of a thesis for my Masters project. I may also use the information in 
conference presentations and publications in professional journats: It is hoped that the research will be beneficial to nurses working in the field of child and adolescent mental health, and both adult mental health nurses and paediatric nurses, who often care for adolescents experiencing mental health problems in an inpatient setting.

Participants will be offered a summary of my findings by ticking the box on the consent form. You will have the right to withdraw from; the research any time. The data obtained from the interviews will bes stored in a locked cupboard for two years after which time it will be destroyed.

If you have any questions regarding participating in this research please don't hesitate to contact me on email Julia.mclean@tdhb.org.nz; or you can call me either at work 067537790 Ext 8872 or my cell phone 0272391156. You could also contact my supervisor Thelma Pudkey at Victoria University on 0800108 005, Ext 6648, or on email Thelma.Puckey@vuw.ac.nz 


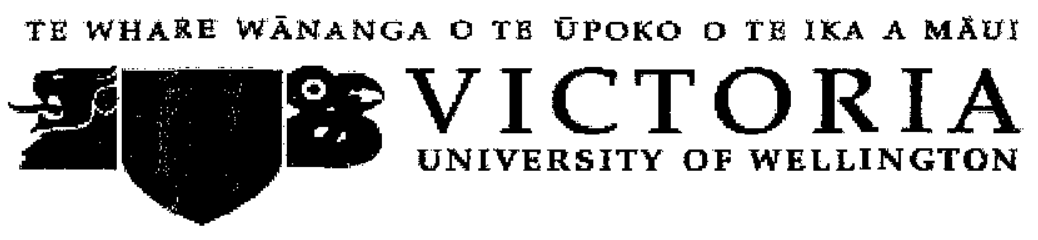

\section{Appendix 3}

\section{Consent Form}

\section{Pushing the Boundaries: Relationships with Adolescents}

\section{CONSENT FORM}

- I agree to participate in the research study "Pushing the boundaries: Relationships with adolescents" as described in the attached information sheet.

- I understand the purpose and nature of this study and am participating voluntarily.

- I have had any questions I have answered to my satisfaction.

- I grant permission for the interview to be audio taped and the datat to be used in the process of completing a Masters degree, including: a thesis and any other future publication or conference presentation.

- lunderstand that my name and other demographic material which may identify me will not be used and that confidentiality will be maintained to the best of the researcher's ability, but that the data obtained may/ be recognisable by my peers.

- I understand I have a right to withdraw my consent and any data; likave given up to two weeks after the interview.

$\square$ I would like a summary of the research findings at the completion of the project

I agree to participate in this study under the conditions set out in the information Sheet.

Signature:

Date:

Full Name - printed 


\section{References}

American Psychiatric Association. (2000). Diagnostic and statisticall manual of mental disorders ( $4^{\text {th }}$ ed.). Washington DC: Author.

Armstrong, M. (2006). Self-harm, young people and nursing. In T. McDougall (Ed.), Child and Adolescent Mental Health NursingOxford, England: Blackwell Publishing Ltd.

Allenbach, A., \& Steinmiller, E. A. (2004). Waiting together: Transleting the principles of therapeutic relationships one step further. Journali of Specialists in Pediatric Nursing, 9(1), 24-32.

Barker, P. (1998). Psychiatric Nursing (2nd ed.). Cheltenham, England: Stanley Thomas.

Barnsteiner, J., \& Gillis-Donovan, J. (1990). Being related and separate: A standard for therapeutic relationships. The American Journal off Maternal Child Nursing, 15, 223-228.

Benner, P. (1991). The role of experience, narrative, and community in skilled ethical compartment. Advances in Nursing Science, 14(2) $1-21$.

Benner, P., \& Wrubel, J. (1989). The primacy of caring: Stress and coping in health and illness. Los Angeles: Addison-Wesley Publisthing Company.

Bruner, E. M. (1984). Text, Play and Story: The construction and reconstruction of self and society. Chicago: Waveland Press Inc.

Bruner, J. (1991). The narrative construction of reality. Critical Inquiry/, $18(1), 1-21$. 
Carey, K. B. (1998). Treatment boundaries in the case management relationship: A behavioral perspective. Community Mental Health Journal, 34(3), 313-317.

Carper, B. A. (1978). Fundamental patterns of knowing in nursing. Advances in Nursing Science, 1(1), 13-23.

Carson, A. M., \& Fairbairn, G. J. (2002). The whole story: Towardss an: ethical research methodology. (Qualitative approaches). Nurse Researcher, 10(1), 15-30.

Casement, P. (1985). On learning from the patient. London: Tavistack: Publications Ltd.

Clandinin, D. J., \& Connelly, F. M. (1989). Teachers as curriculum planners: Narratives of experience. New York: Teachers College Press.

Clandinin, D. J., \& Connelly, F. M. (1990). Stories of experience and narrative inquiry. Educational Researcher, 19(5), 2-14.

Clandinin, D. J., \& Connelly, F. M. (1991). Narrative and story in practice and research. in D. A. Schon (Ed.), The reflective turn: Case studies in and on educational practice. New York: Teachers College Press.

Clandinin, D. J., and Connelly, F. M. (2000). Narrative inquiry: Experience and story in qualitative research. San Francisco: JosseyBass inc.

Clandinin, D. J., Huber, J., Huber, M., Murphy, M. S., Murray Orr, A, Pearce, M., et al. (2006). Composing diverse identities: Narrative inquiries into the interwoven lives of children and teachers. New York: Routledge. 
Cleary, M. (2003). The challenges of mental health care reform for contemporary mental health nursing practice: Delivery of nursings care. International Journal of Mental Health Nursing, 12, 213-222.

Croghan, R., Griffin, C., Hunter, J., \& Phoenix, A. (2006). Style failure: Consumption, identity and social exclusion. Journal of Youth Studies, 9(4), 463-478.

Crotty, M. (1998). The foundations of social research: Meaning and? perspective in the research process. Sydney, Australia: Allen \& Unwin Pty Ltd.

Curtis, L., \& Hodge, M. (1994). Old standards, new dilemmas: ethies and boundaries in community support services. Psychosocial Rehabilfation Journal, 18(2), 13-33.

Cutcliffe, J. R. (1997). The nature of expert psychiatric nurse practice: a grounded theory study. Journal of Clinical Nursing, 6, 325-332.

Denzin, N. K. (1989). Interpretative interactionism (Vol. 16). Londarte: Sage Publications, Inc.

Dewey, J. (1938). Experience and education. New York: The Mactifllan Company.

de Klerk, (2005). Slang and swearing as markers of inclusion and exclusion in adolescence. In A. Williams \& C. Thurslow (Eds.), Talking adolescence: Perspectives on communication in the teenage ywars (3rd ed.). New York: Peter Lang.

de Raeve, L. (1998). Knowing patients: how much and how well? Barnes,P. Griffith, J. Ord \& D. Wells (Eds.), Face to face with distitess; 
the professional use of self in psychosocial care. Oxford, England: Butterworth-Heinemann.

Drury, J. (2005). Young people's communication with adults in then institutional order. In A. Williams \& C. Thurslow (Eds.), Talking adolescence: Perspectives on communication in the teenage years (3rd ed.). New York: Peter Lang:

Durie, M. (1995). Nga Matatini Maori diverse maori realties. Warkganga purongo korerorero, 14-17.2.95, 1-10.

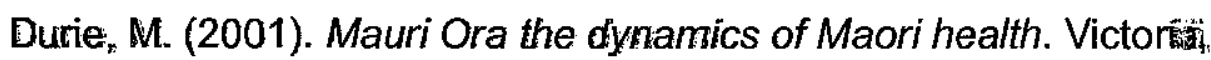
Australia: Oxford University Press.

Durie, M. (2003). Nga Kahui Pou launching Maori futures. Wellinton, New Zealand: Huia Publishers.

Elkind, D. (1984). All grown up and no place to go: Teenagers irricrisis. Landon: Addison-Wesley Publishing Company.

Elkind, D. (1998). Cognitive Development. In S. Friedman, M. Fisker, S. Schonberg \& E. Alderman (Eds.) $)_{x}$ Comprehensive adolescent healthcare (2nd ed.). New York: Mosby.

Emden, C. (1998). Theoretical perspectives on narrative inquiry. Collegian, 5(2), 30-35.

Erikson, E. H. (1963). Childhood and society (2nd ed.). New Yortit WW Norton \& Company.

Erikson, E. H. (1968). Identity: Youth and crisis. London: Faber terFaber Limited.

Ford, K, \& Turner, d. (2001). Stories seldom told: paediatric nur ses' 
experiences of caring for hospitalised children with special needss and their families. Journal of Advanced Nursing, 33(3), 288-295.

Frankel, R. (1998). The adolescent psyche. London: Rutledge.

Gastmans, C. (1998). Interpersonal relations in nursing: a philosophiicalethical analysis of the work of Hilderguard E Peplau. Journal of Advanced Nursing, 28(6), 1-11.

Geanellos, R. (1997). Explicating practice knowledge: A hermenentic inquiry into adolescent mental health nursing. Unpublished Degree of Doctor of Philosophy, Australian Catholic University, Ascot Vale Victoria.

Geanellos, R. (1998). Hermeneutic philosophy. Part II: a nursing research example of the hermeneutic imperative to address forestructures/preunderstandings. Nursing Inquiry, 5, 239-247.

Geanellos, R. (1999). A review of the sociopolitical context of adolescent mental health and adolescent mental health nursing in Australia. Australian and New Zealand Journal of Mental Health Nursing, 8, 134142.

Geanellos, R. (2002). Transformative change of self: The unique tocus of(adolescent) mental health nursing? International Journal of Mental Health Nursing, 11, 174-185.

Geanellos, R. (2003). Understanding the need for personal space boundary restoration in women-client survivors of interfamilial childhood sexual abuse. International Journal of Mental Health Nursing, 12, 186-193.

Geanellos, R. (2004). Nursing based evidence: moving beyond evidence-based practice in mental health nursing. Journal of 
Evaluation in Clinical Practice, 10(2), 177-186.

Geanellos, R. (2005). Sustaining well-being and enabling recovery: The therapeutic effect of nurse friendliness on clients and nursing environments. Contemporary Nurse, 19, 242-252.

Griffiths, P., \& Leach, G. (1998). Psychosocial nursing: a model learnt from experience. In E. Barnes, Griffith, P., Ord, J., Wells, D. (Ed. Face to face with distress: The professional use of self in psychological care (pp. 5-41). Oxford, England: Butterworth-Heinemann.

Gudmundsdottir, S. (1995). The narrative nature of pedagogical content knowledge. In H. McEwan \& K. Egan (Eds.), Narrative in teachinge. learning and research. New York: Teachers College Press.

Halton, W. (1994). Some unconscious aspects of organisational lifer contributions from psychoanalysis. In A. Obholzer \& V. Roberts, $\mathbb{Z}$ (Eds.), The unconscious at work: Individual and organisational stess in the human services. London: Routledge.

Hamburg, B. (1998). Psychosocial development. In S. Friedman, Fisher, S. Schonberg \& E. Alderman (Eds.), Comprehensive adolescent healthcare ( $2^{\text {nd }}$ ed.). New York: Mosby.

Hawes, R. (2005). Therapeutic relationships with families: Rebecca Hawes set out to identify the features of ideal therapeutic relationships in children's cancer nursing and discovered lessons for all nurses working with children, young people and families. (Clinical). Paediatric Nursing, 17(6), 15-19.

Health Research Council of New Zealand. (1998). Guidelines for researchers on health research involving Maori. Auckland: Health Research Council of New Zealand. 
Hendry, L., \& Ktoep, M. (2005). 'Talkin', doin' and bein' with friendsi

Leisure and communication in adolescents. In A. Williams \& C.

Thurslow (Eds.), Talking adolescence: perspectives on communitiation in the teenage years (3rd ed.). New York: Peter Lang.

Higgins, I., \& McDougall, T. (2006). Nursing children and adolescents who are aggressive or violent: a psychological approach. In $\mathrm{T}$. McDougall (Ed.), Child and Adolescent Mental Health Nursing. Oxford, England: Blackwell Publishing Ltd.

Hinds, P. S. (1988). The relationship of nurses' caring behaviours with hopefulness and health care outcomes in adolescents. Archives of Psychiatric Nursing, 2(1), 21-29.

Hodgkinson, P., Watson, E., Wadge, M., Abeles, P., Danquah, A. (2005). Dependency scoring for child and adolescent inpatient mental health services. Paediatric Nursing, 17(10), 18-22.

Horberg, U., Brunt, D., and Axelsson, A. (2004). Clients' perceptions: of client-nurse relationships in local authority psychiatric services: $A$ qualitative study. International Journal of Mental Health Nursing, 13, 917.

Johansson, H., \& Eklund, M. (2003). Patients' opinion on what constitutes good psychiatric care. Scandinavian Journal of Caring Science, $17,339-346$.

Kimberly, A. (2002). Caring for adolescents in the adult intensive care: unit (Pediatrics). Critical Care Nurse, 22(2), 80-97.

Koch, T. (1998). Story telling: is it really research? Journal of Advariced Nursing, 28(6), 1182-1190.

KPMG Consulting, Health, Education and Community Servịces. (2001i). 
KPMG Strategic Review of Undergraduate Nursing Education: For: Nursing Council New Zealand.

Krawitz, R., \& Watson, C. (1999). Borderline personality disorder: pathways to effective service delivery and clinical treatment options. Wellington, New Zealand: Mental Health Commission.

Lieblich, A., \& Josselson, R. (1997). The narrative study of lives (Vell 5).Thousand Oaks: Sage Publications.

Lincoln, Y. S.s \& Guba, E. G. (1985). Naturalistic inquiry. London: Sage Publications.

Lofland, J. (1971). Analyzing social settings. California: Wadsworth Publishing Company, Inc.

McCance, T. V., McKenna, H. P., \& Boore, J. R. P. (2001). Exploring caring using narrative methodology: An analysis of the approach. Journal of Advanced Nursing, 33(3), 350-356.

McCann, T. V., \& Clark, E. (2003). A grounded theory study of the that nurses play in increasing clients' willingness to access community mental health services. International Journal of Mental Health Nursing, $12,279-287$.

McCutcheon, H. I., \& Pincombe, J. (2001). Intuition: an important tool in the practice of nursing. Journal of Advanced Nursing, 35(3), 342-348.

McMahon, L., \& Ward, A. (1998). Helping and the personal response. In W. A \& M. L (Eds.), Intuition is not enough; matching learning with practice in therapeutic child care. London: Routledge.

Mishler, E. G. (1986). Research interviewing; context and narrative. London: Harvard University Press. 
Mitchell, G. (2001) A qualitative study exploring how qualified mental! health nurses deal with incidents that conflict with their accountability. Journal of Psychiatric and Mental Health Nursing. 8 241-248.

Morse, J. M., \& Field, P. A. (1995). Qualitative research methods health professionals (2nd ed.). London: Sage Publications.

Moyle, W. (2003). Nurse-patient relationship: A dichotomy of expectations. International Journal of Mental Health Nursing, 12, 103109.

Murray, B. L., \& Wright, K. (2006). Integration of a suicide risk assessment and intervention approach; the perspective of youth. Journal of Psychiatric and Mental Health Nursing, 13, 157-164.

Noller, P. (2005). Communication with parents and other family members: The implications of family process for young people's wellbeing. In A. Williams \& C. Thurslow (Eds.), Talking adolescence: Perspectives on communication in the teenage years (3rd ed.). New York: Peter Lang.

Nursing Council of New Zealand (2005). Competencies for the registered nurse scope of practice: Nursing Council of New Zealand.

Obholzer, A., \& Roberts, V., Z. (1994). The troublesome individuat and the troubled institution. In A. Obholzer \& V. Roberts, Z (Eds.), The unconscious at work; individual and organisational stress in the luman services. London: Routledge.

O'Brien, A. (1999). Negotiating the relationship: Mental health nurses' perceptions of their practice. Australian and New Zealand Journat of Mental Health Nursing, 8, 153-161. 
O'Brien, L. (2000). Nurse-client relationships: The experience of community psychiatric nurses. Australian and New Zealand Journal of Mental Health Nursing, 9, 184-194.

Ollernshaw, J. A., \& Creswell, J. W. (2002). Narrative research: A comparison of two restorying data analysis approaches. Qualitative inquiry, 8(3), 329-339.

Ord, J. (1998). Subjective experience and social enquiry. In E. Bames; Griffith, P., Ord, J., Wells, D. (Ed.), Face to face with distress: The professional use of self in psychological care (pp. 42-52). Oxford. England: Butterworth-Heinemann.

Overcash, J. A. (2004). Narrative research: a viable methodology for clinical nursing. Nursing Forum, 39(1), 15-23.

Paget, M. A. (1983). Experience and knowledge. Human Studies, 6, 6790.

Peplau, H., E. (1952). Interpersonal relations in nursing; a conceptual frame of reference for psychodynamic nursing. New York: G. P. Putnam Sons.

Peplau, H., E. (1962). Interpersonal techniques: The crux of psychiatiric nursing. American Journal of Nursing, 62, 50-54.

Peplau, H., E. (1988). Interpersonal relations in nursing (2nd ed.). London: Macmillan.

Peplau, H. E. (1997). Peplau's theory on interpersonal relations. Nursing Science Quarterly, 10(4), 162-167.

Peternelj-Taylor, C. (2002). Professional boundaries: A matter of therapeutic integrity. Journal of Psychosocial Nursing \& Mental Health 
Sciences, 40(4), $22-43$.

Peters, J. (2003). Undergraduate training in child and adolescent mental health: A review. Auckland, New Zealand: The Werry Centre for Child and Adolescent Mental Health, The University of Auckland.

Piaget, J., \& Inhelder, B. (1969). The psychology of the child. Londion: Routledge \& Kegan Paul.

Polit, D. F., \& Beck, C. T. (2006). Essentials of nursing research: methods, appraisal, and utilisation (Sixth ed.). Philadelphia: Lippineott Williams \&Wilkins.

Polkinghorne, D. E. (1995). Transformative narratives: From victimic: to agentic life plots. The American Journal of Occupational Therapys. 50(4), 299-305.

Prebble, K. (2001). On the brink of change? Implications for the review of undergraduate education in New Zealand for Mental Health Nurses. Australian and New Zealand Journal of Mental Health Nursing, 13 , 134-142.

Ramage, C., Bir, J., Towns, A., Vague, R., Cargo, T., \& Niumata-Falkafa, M. (2005). Stocktake of child and adolescent mental health services in New Zealand. Auckland: The Werry Centre for child \& adolescente mental health workforce development, The University of Auckland.

Ramsden, I. (2000). Cultural safety / Kawa Whakaruruhau Ten years on: a personal overview. Nursing Praxis in New Zealand; 15(1), 4-12..

Ramjan, L. M. (2004). Nurses and the 'therapeutic relationship'; caring for adolescents with anorexia nervosa. Journal of Advanced Nursing; 45(5), 495-503. 
Riessman ${ }_{x}$ C. K. (1993). Narrative analysis. Newbury Park: Sage Publications.

Roberts, K., \& Taylor, B. (1998). Nursing research processes: an Australian perspective. South Melbourne, Australia: Nelson international Thomson Publishing Company.

Roberts, V., Z. (1994). The organisation of work. In A. R. Obholzer, $V_{n}^{\prime} Z$. (Ed.), The unconscious at work; individual and organisational stressin human services (pp. 29-38). London: Routledge.

Rogers, C. (1980). A way of being. Boston: Houghton Mifflin Company?

Sandelowski, M. (1986). The problem of rigor in qualitative researcht. Advances in Nursing Science, 8(3), 27-37.

Sandelowski, M. (1991). Telling stories: Narrative approaches in qualitative research. Journal of Nursing Scholarship, 23(3), 161-166.

Sandelowski, M. (1993). Rigor or rigor mortis: The problem of rigor in qualitative research revisited. Advances in Nursing Science, 16(2) $)$ 1i-8.

Scanlon, A. (2006). Psychiatric nurses perceptions of the constituents of the therapeutic relationship: a grounded theory study. Journal of Psychiatric and Mental Health Nursing, 13, 319-329.

Schneider, Z., Elliott, D., Beanland, C., LoBiondo-Wood, G., \& Haher, J. (Eds.). (2003). Nursing research methods, critical appraisal and utilisation (2nd ed.). Marrickville: Mosby.

Sharland, E. (2006). Young people, risk taking and risk making: sorne: thoughts for social work. British Journal of Social Work, 36, 247-265i.

Sheets, V. (1999). Professional interpersonal boundaries: A 
commentary. Pediatric Nursing, 25(6), 657-662.

The Australian Nursing and Midwifery Council, Nursing Council of New Zealand and Faculty of Health, University of Newcastle. (2007). A discussion paper: ANMC National guidelines on boundaries of professional practice for nurses and midwives. Author.

The Werry Centre: home: teaching : post graduate teaching : The University of Auckland. (2004). Retrieved September 2, 2004, from http://www.werrycentre.org.nz/?t=92.

Totka, J. P. (1996). Exploring the boundaries of pediatric practice: Wurse stories related to relationships. Pediatric Nursing, 22(3), 191-196.

Walsh, C. (1997). Negotiating the difference in mental health nursing in New Zealand. In S. Tilley (Ed.), The mental health nurse, views off practice and education (pp. 172-185). London: Blackwell Science.

Walsh, C. (2002). Reclaiming our space: reflections on psychiatric mental health nursing from the past to the future. In E. Papps (Edo ) Nursing in New Zealand: Critical issues, different perspectives. Auckland: Pearson Education New Zealand Limited.

Weissman, J. (1995). The therapeutic aspects of acceptance. Perspectives in Psychiatric Care, 31(1), 19-23.

Williams, J., \& Swartz, M. (1998). Treatment boundaries in the case management relationship: A clinical case and discussion. Commenity Mental Health Journal, 34(3), 299-311.

Witherell, C. S. (1995). Narrative landscapes and the moral imagination: Taking the story to heart. In H. McEwan \& K. Egan (Eds.), Narrafive in teaching, learning, and research. New York: Teachers College Press. 
Williams, A., \& Thurstow, C. (2005). Talking adolescence: Perspectives on communication in the teenage years (3rd ed.). New York: Peter Lang.

Woad, P. J., \& Giddings, L. S. (2003). Revealing storied lives - life story narrative inquiry in nursing and midwifery: An interview with Rose McEldowney. Nursing Praxis in New Zealand, 19(2), 4-17.

Worden, M. (1991). Adolescents and their families: an introduction to assessment and intervention. Newt York: The Haworth Press. 
